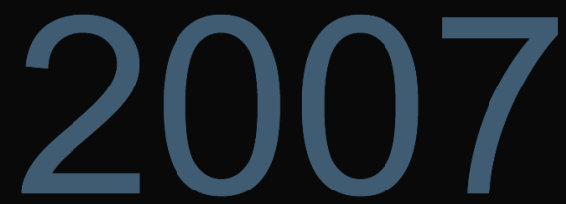

\title{
Kansas City Plant Annual IIIness and Injury Surveillance Report
}

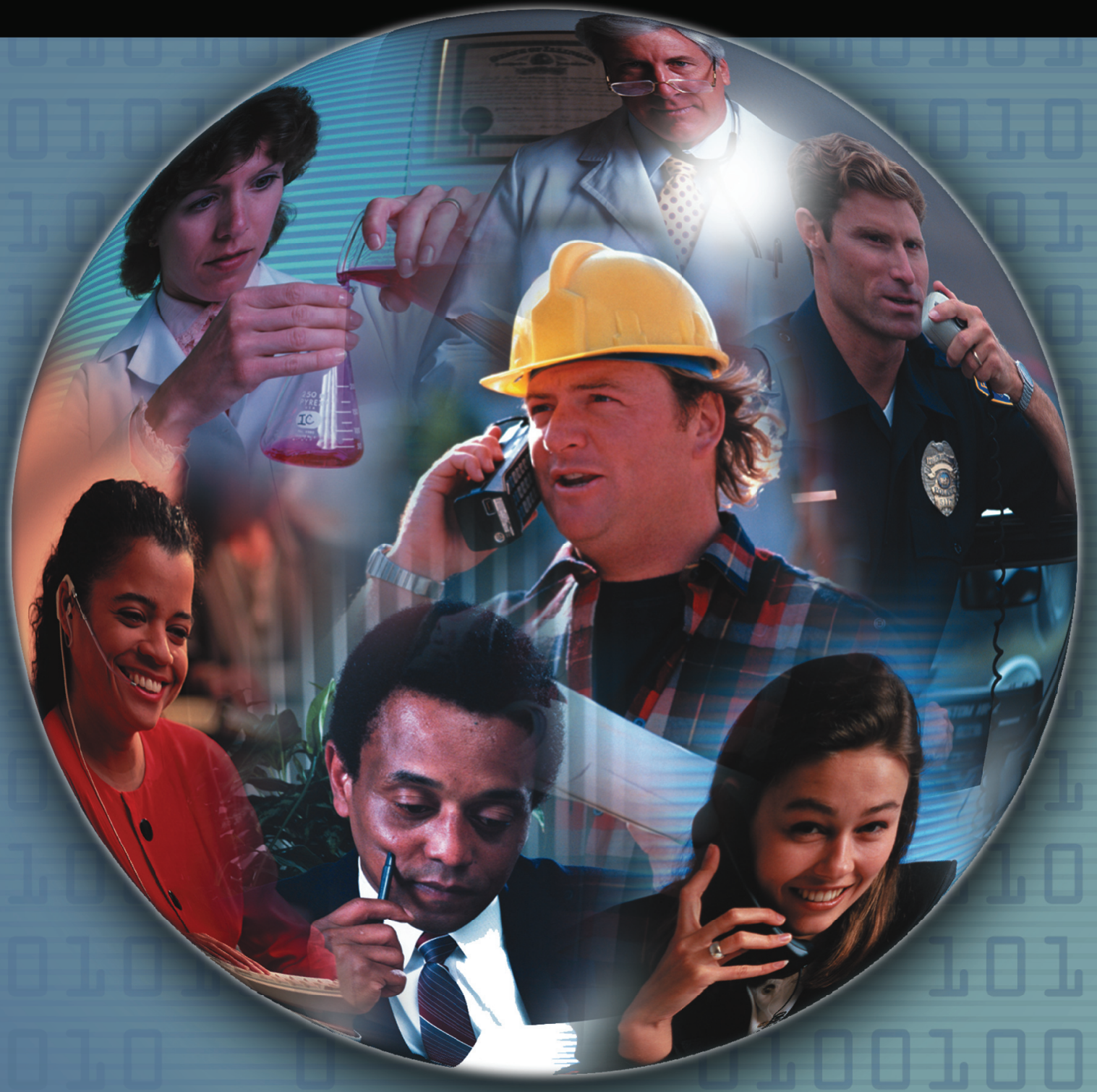




\section{Kansas City Plant \\ 2007 Illness and Injury Surveillance Report}

Questions or comments about this report or the Illness and Injury Surveillance Program (IISP) may be directed to:

E-mail:

Dr. Cliff Strader at cliff.strader@hq.doe.gov or Dr. Bonnie Richter at bonnie.richter@hq.doe.gov

or direct letters to:

Mail Stop HS-13 / 270CC

U.S. Department of Energy

1000 Independence Avenue, S.W.

Washington, DC 20585-0270

Additional information about the Department of Energy's Office of Illness and Injury Prevention Programs, the IISP, and annual reports for DOE sites participating in this program can be found at:

\section{http://www.hss.energy.gov/healthsafety/WSHP/epi/surv/}

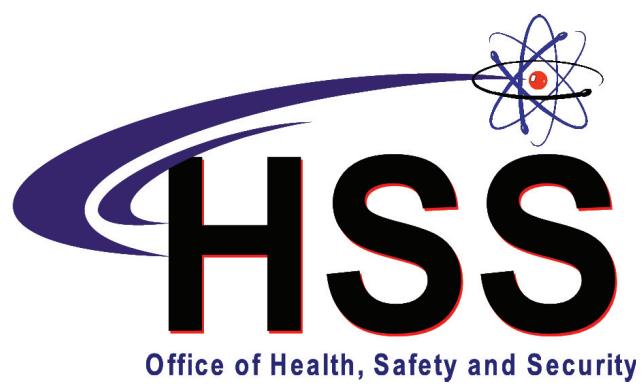

ACKNOWLEDGEMENT

LifeART images copyright 2000 Lippincott Williams \& Wilkins. All rights reserved.

This document was produced under contract number DE-AC05-06OR23100 between the

U.S. Department of Energy and Oak Ridge Associated Universities. 


\section{Kansas City Plant \\ 2007 Illness and Injury Surveillance Report \\ At A Glance}

Since KCP began participating in the DOE Illness and Injury Surveillance Program (IISP), the size of the work force has decreased 17 percent, from 3,170 in 2002 to 2,621 in 2007. There were 648 (25 percent) women and 1,973 (75 percent) men in the work force in 2007 . The ratio of women to men has been constant since 2002 and is similar to the ratio seen among all DOE IISP sites.

Workers in the Professional group had the lowest absence rate among women and Crafts workers had the lowest rate among men. Women in the Security and Fire group and men in the Service category had the highest absence rates.

Women lost 1,975 calendar days due to injury and illness. Musculoskeletal conditions (27 percent), respiratory conditions (14 percent), injuries (12 percent), and unspecified symptoms (12 percent) accounted for 65 percent of all reported diagnoses among women.

Men lost 3,411 calendar days due to injury and illness. Fifty-six percent of all reported diagnoses among men were due to musculoskeletal conditions $(22$ percent), injuries (17 percent), and digestive conditions (17 percent).

Among women and men, the rate of illness and injuries increased with age. This same trend is seen across the DOE complex among IISP sites.

Among women, the rate of all diagnoses combined, which increased 77 percent from 2003 to 2006, decreased to near the 2003 rate in 2007. Among men, the rate, which began to decrease in 2006 , continued to decrease in 2007 . The decrease in the rates for men and women was not due to any particular diagnostic category but rather a decrease in many categories.

The 2007 age-adjusted rates for all diagnoses increased among Professional, Technical Support, and Security and Fire groups for men compared with 2006. Among women, the 2007 rates declined in all occupational groups compared with 2006.

Ten diagnoses for sentinel occupational health events were reported. Carpal tunnel syndrome accounted for 8 of these diagnoses. The other 2 diagnoses were for contact dermatitis and fractured femur. The carpal tunnel diagnoses were reported by workers from 6 of the 7 occupational groups. 
Thirteen OSHA-recordable events among men and 9 OSHA-recordable events among women produced an event rate of 1 per 100 workers for both men and women in 2007.

Injuries accounted for almost half (49 percent) of the diagnoses. Falls and overexertion and strenuous movements were the most frequent types of accidents.

OsHA events were responsible for 27 days lost and 168 days of restricted duty in 2007. Four falls recorded among Technical Support and Administrative Support workers produced 78 percent of the lost workdays and 42 percent of the days on restricted duty. 
The Kansas City Plant Work Force - 2007

The Work Force by Gender and Age 1

The Work Force by Gender and Job

Category....

\section{Number and Length of Absences}

Absence Rate by Gender and Age

Number of Days Absent by

Gender and Age... $\ldots 2$

Absence Rate by Job Category

and Gender.

Average Duration of Absence by

Job Category and Gender.

\section{Diagnostic Categories}

Number of Diagnoses and Lost Calendar Days by Diagnostic Category

(Categorized by ICD-9-CM) and Gender......4

Common Diagnoses Among Female

Workers in 2007

Common Diagnoses Among Male

Workers in 2007

Number of Most Frequently Reported

Diagnoses by Job Category and Gender ......7

\section{Rates of Disease Occurrence}

Rates for All Illnesses and Injuries Combined by Job Category, Gender, and Age

Rates for Selected Diagnostic Categories by Job Category, Gender, and Age.

\section{Time Trends}

Age-Adjusted Rates for All Diagnoses Combined Among Women and Men from 2003 to 2007 . 10
Age-Adjusted Rates for Selected Diagnostic Categories Among Women and Men from 2003 to 2007 ...................................... 11

Age-Adjusted Rates for All Diagnoses Combined Among Women and Men by Job Category from 2003 to 2007 .... 12

\section{Sentinel Health Events for Occupations (SHEOs)}

Characteristics of SHEOs by Gender.... 13

SHEO Diagnoses by Gender .

Occupational Safety and Health Administration (OSHA)-Recordable Events

OSHA-Recordable Events by Gender and Age 14

OSHA-Recordable Events by Job

Category and Gender 14

\section{Diagnostic and Accident Categories for OSHA-Recordable Events}

OSHA-Recordable Diagnoses by

Diagnostic Category and Gender ... 15

OSHA-Recordable Accidents by Type and Gender. 15

\section{Rates of OSHA-Recordable Events}

OSHA-Recordable Rates by Age and Job Categories Among Women, All Diagnoses Combined

OSHA-Recordable Rates by Age and Job Categories Among Men, All Diagnoses Combined .16

\section{Time Trends for OSHA-Recordable Events}

Age-Adjusted Rates for All OSHA-Recordable Diagnoses Combined Among Women and Men by Job Category from 2003 to 2007 ... 17

\section{Appendices}

Appendices A-W. 19 
The Kansas City Plant Work Force - 2007

Figure 1. The Work Force by Gender and Age

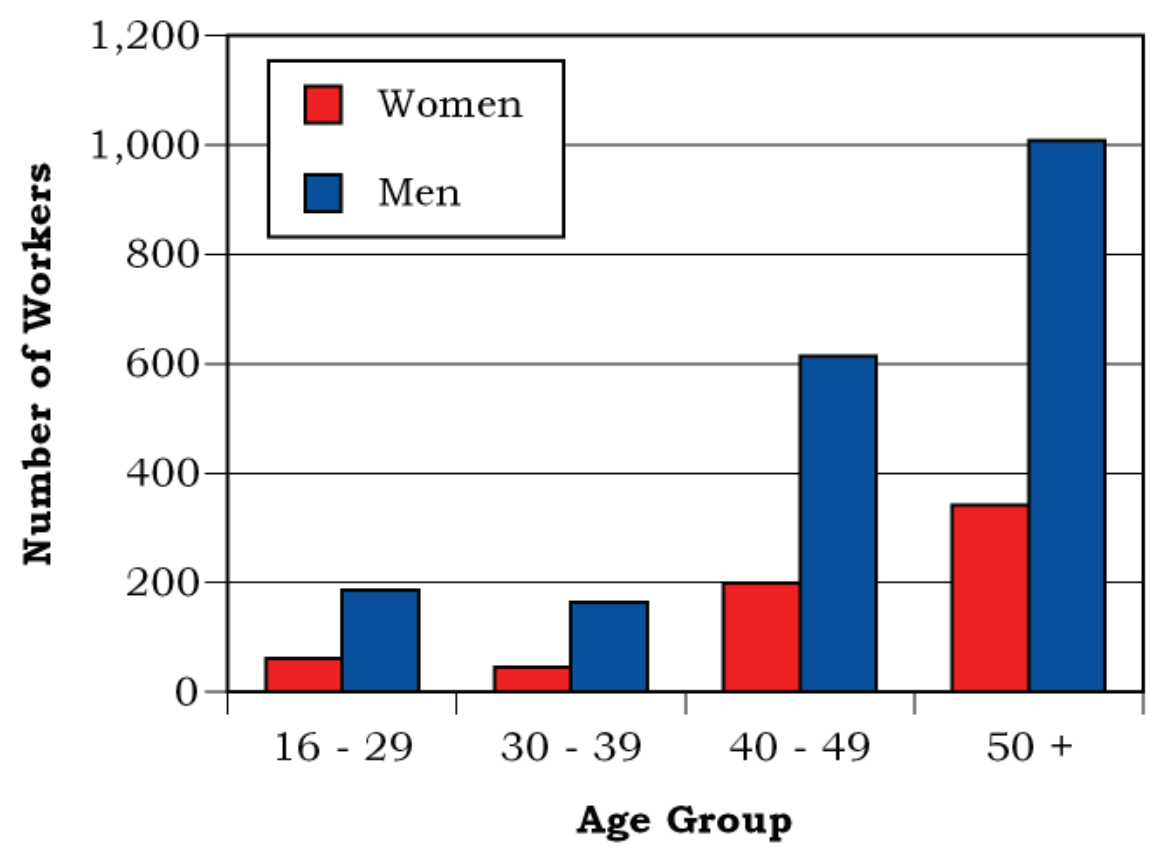

Figure 2. The Work Force by Gender and Job Category

\begin{tabular}{|l|c|c|}
\hline \multirow{2}{*}{ Job Category } & Women & Men \\
\hline \multirow{2}{*}{ Professional } & 141 & 832 \\
& $22 \%$ & $42 \%$ \\
\hline \multirow{2}{*}{ Administrative Support } & 262 & 269 \\
& $40 \%$ & $14 \%$ \\
\hline \multirow{2}{*}{ Technical Support } & 63 & 279 \\
\hline \multirow{2}{*}{ Service } & $10 \%$ & $14 \%$ \\
\hline \multirow{2}{*}{ Security and Fire } & 43 & 61 \\
\hline \multirow{2}{*}{ Crafts } & $6 \%$ & $3 \%$ \\
\hline \multirow{2}{*}{ Line Operators } & 12 & 66 \\
& $2 \%$ & $4 \%$ \\
\hline \multirow{2}{*}{ Total } & 19 & 342 \\
& $3 \%$ & $17 \%$ \\
\hline
\end{tabular}


Number and Length of Absences

Figure 3. Absence Rate by Gender and Age

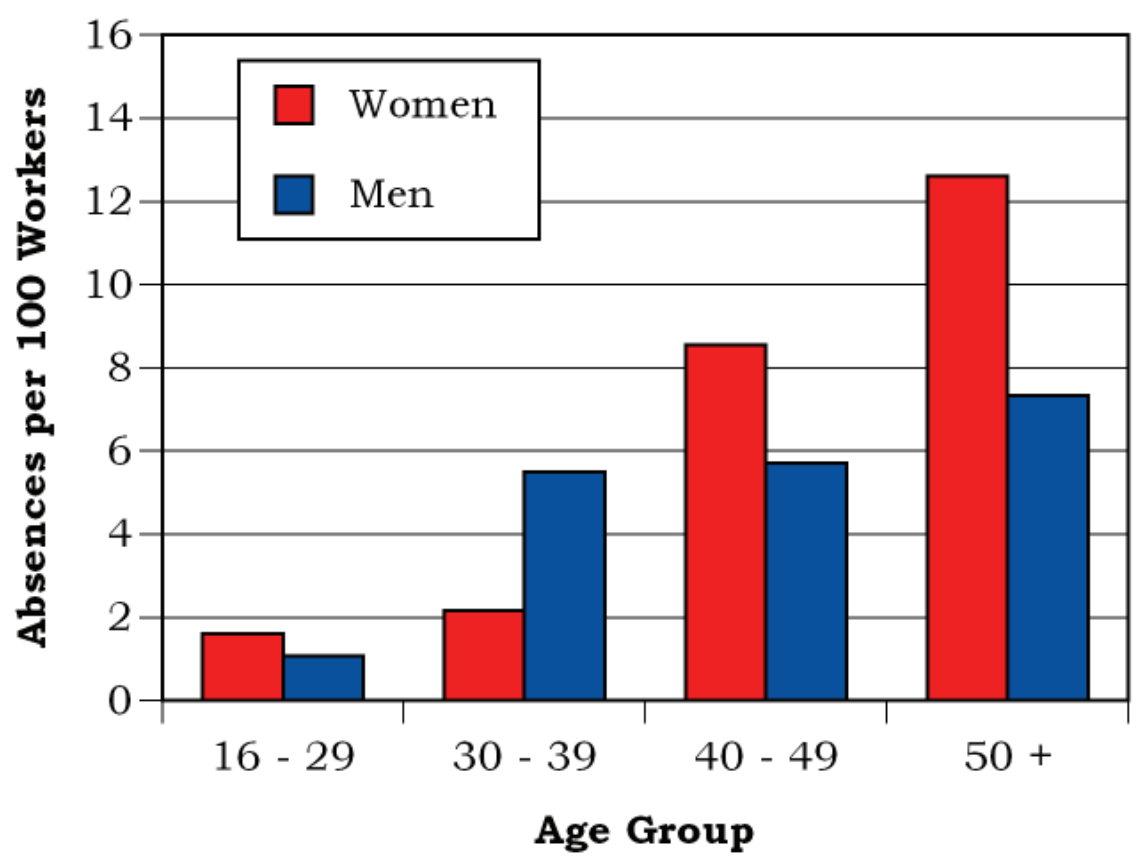

Figure 4. Number of Days Absent by Gender and Age

\begin{tabular}{|c|c|c|c|c|}
\hline \multirow{3}{*}{ Gender } & \multirow{2}{*}{ Age } & Number of & \multicolumn{2}{|c|}{ Number of Days Absent } \\
\cline { 3 - 5 } & & Absences & Total & Average \\
\hline \multirow{4}{*}{ Women } & $16-29$ & 1 & 18 & 18 \\
\cline { 2 - 5 } & $30-39$ & 1 & 3 & 3 \\
\cline { 2 - 5 } & $40-49$ & 17 & 229 & 13 \\
\cline { 2 - 5 } & $50+$ & 43 & 1,725 & 40 \\
\cline { 2 - 5 } & Total & 62 & 1,975 & 32 \\
\hline \multirow{4}{*}{ Men } & $16-29$ & 2 & 15 & 8 \\
\cline { 2 - 5 } & $30-39$ & 9 & 116 & 13 \\
\cline { 2 - 5 } & $40-49$ & 35 & 924 & 26 \\
\cline { 2 - 5 } & $50+$ & 74 & 2,356 & 32 \\
\cline { 2 - 5 } & Total & 120 & 3,411 & 28 \\
\hline
\end{tabular}


Figure 5. Absence Rate by Job Category and Gender

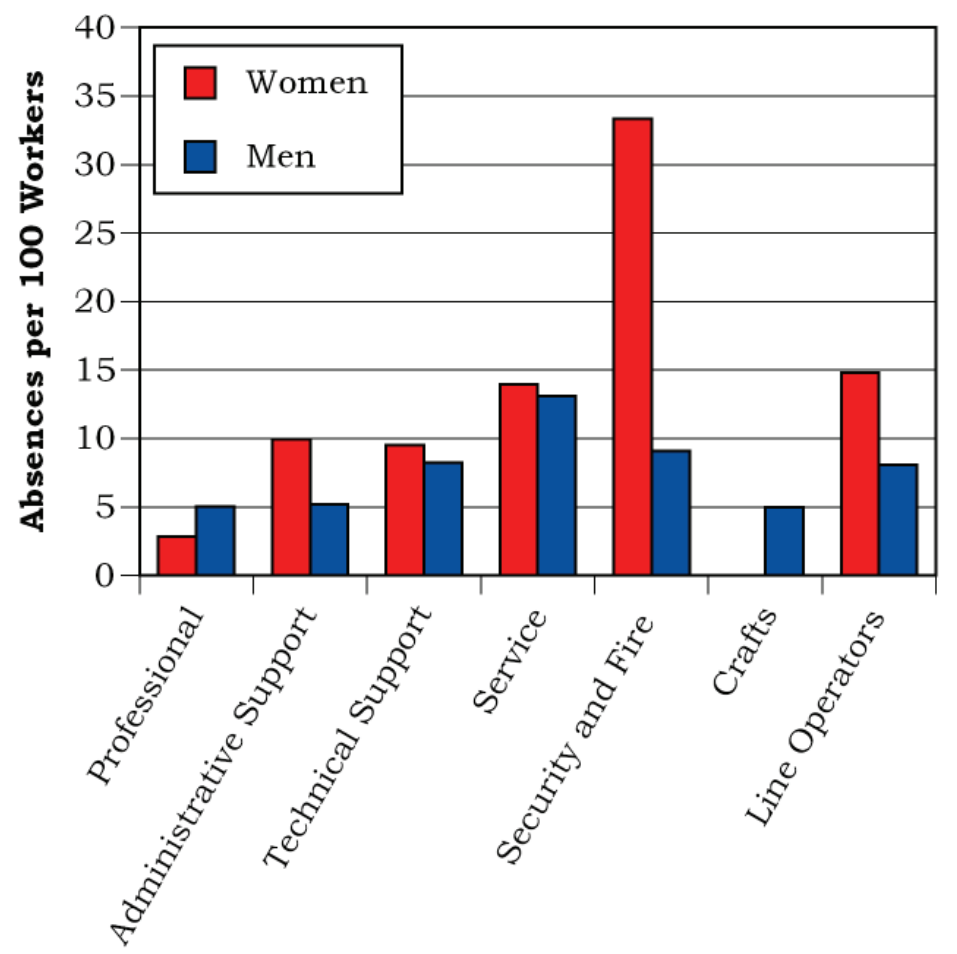

Job Category

Figure 6. Average Duration of Absence by Job Category and Gender

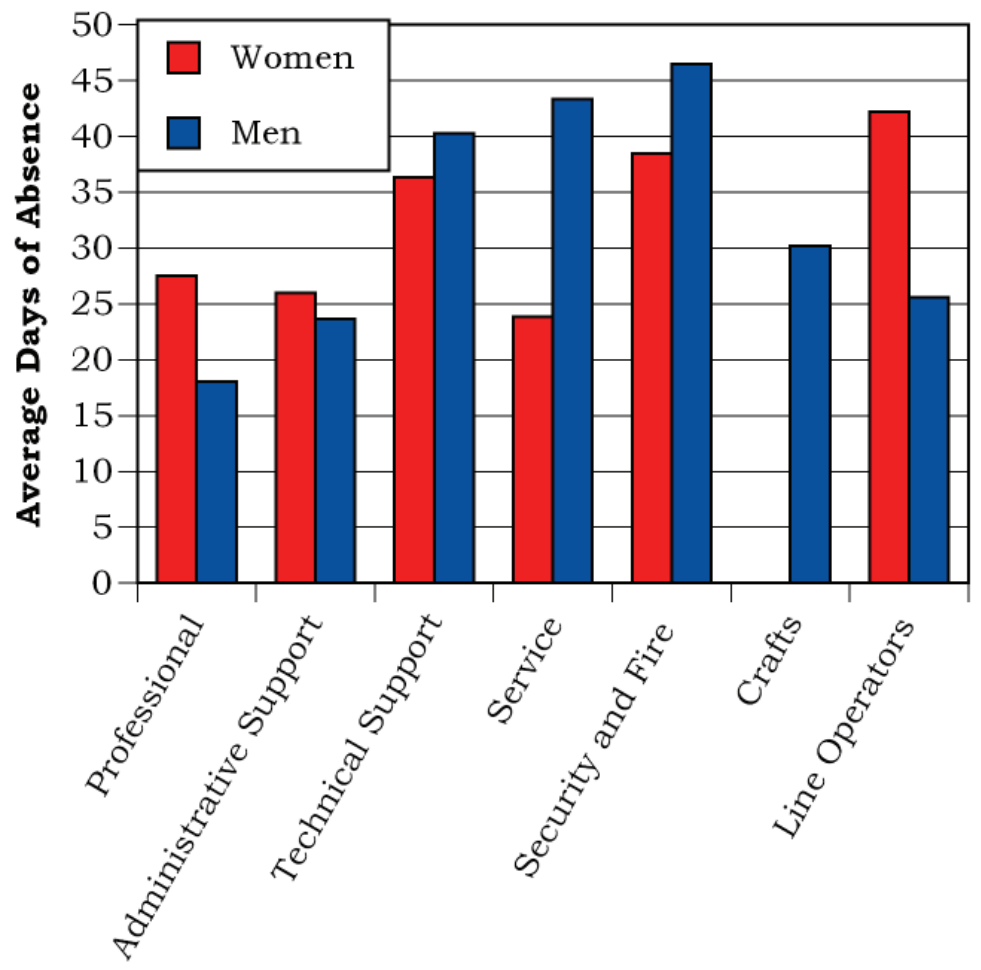

Job Category 


\section{Diagnostic Categories}

\section{Figure 7. Number of Diagnoses and Lost Calendar Days by Diagnostic Category} (Categorized by ICD-9-CM) and Gender

\begin{tabular}{|c|c|c|c|c|}
\hline \multirow[b]{2}{*}{ Diagnostic Category } & \multicolumn{2}{|c|}{ Women } & \multicolumn{2}{|c|}{ Men } \\
\hline & $\begin{array}{l}\text { Number of } \\
\text { Diagnoses }\end{array}$ & $\begin{array}{c}\text { Number } \\
\text { of Lost } \\
\text { Calendar } \\
\text { Days }\end{array}$ & $\begin{array}{l}\text { Number of } \\
\text { Diagnoses }\end{array}$ & $\begin{array}{c}\text { Number } \\
\text { of Lost } \\
\text { Calendar } \\
\text { Days }\end{array}$ \\
\hline Benign Growths & 1 & 15 & 1 & 31 \\
\hline Blood & 2 & 72 & 3 & 166 \\
\hline Cancer & 0 & 0 & 5 & 89 \\
\hline Digestive & 1 & 10 & 32 & 511 \\
\hline Endocrine/Metabolic & 2 & 32 & 3 & 241 \\
\hline Existing Birth Condition & 1 & 20 & 1 & 17 \\
\hline Genitourinary & 7 & 122 & 7 & 75 \\
\hline Heart/Circulatory & 7 & 164 & 17 & 679 \\
\hline Infections/Parasites & 1 & 31 & 2 & 42 \\
\hline Injury & 12 & 562 & 32 & 897 \\
\hline Miscarriage & 0 & 0 & NA & NA \\
\hline Musculoskeletal & 27 & 1,083 & 42 & 1,243 \\
\hline Nervous System & 6 & 359 & 8 & 204 \\
\hline Psychological & 1 & 17 & 3 & 48 \\
\hline Respiratory & 14 & 139 & 6 & 95 \\
\hline Skin & 5 & 129 & 3 & 13 \\
\hline Unspecified Symptoms & 12 & 317 & 22 & 436 \\
\hline
\end{tabular}

Note: Lost calendar days for each absence are counted more than once when multiple diagnoses occur in different diagnostic categories for the same absence. 
Figure 8. Common Diagnoses Among Female Workers in 2007

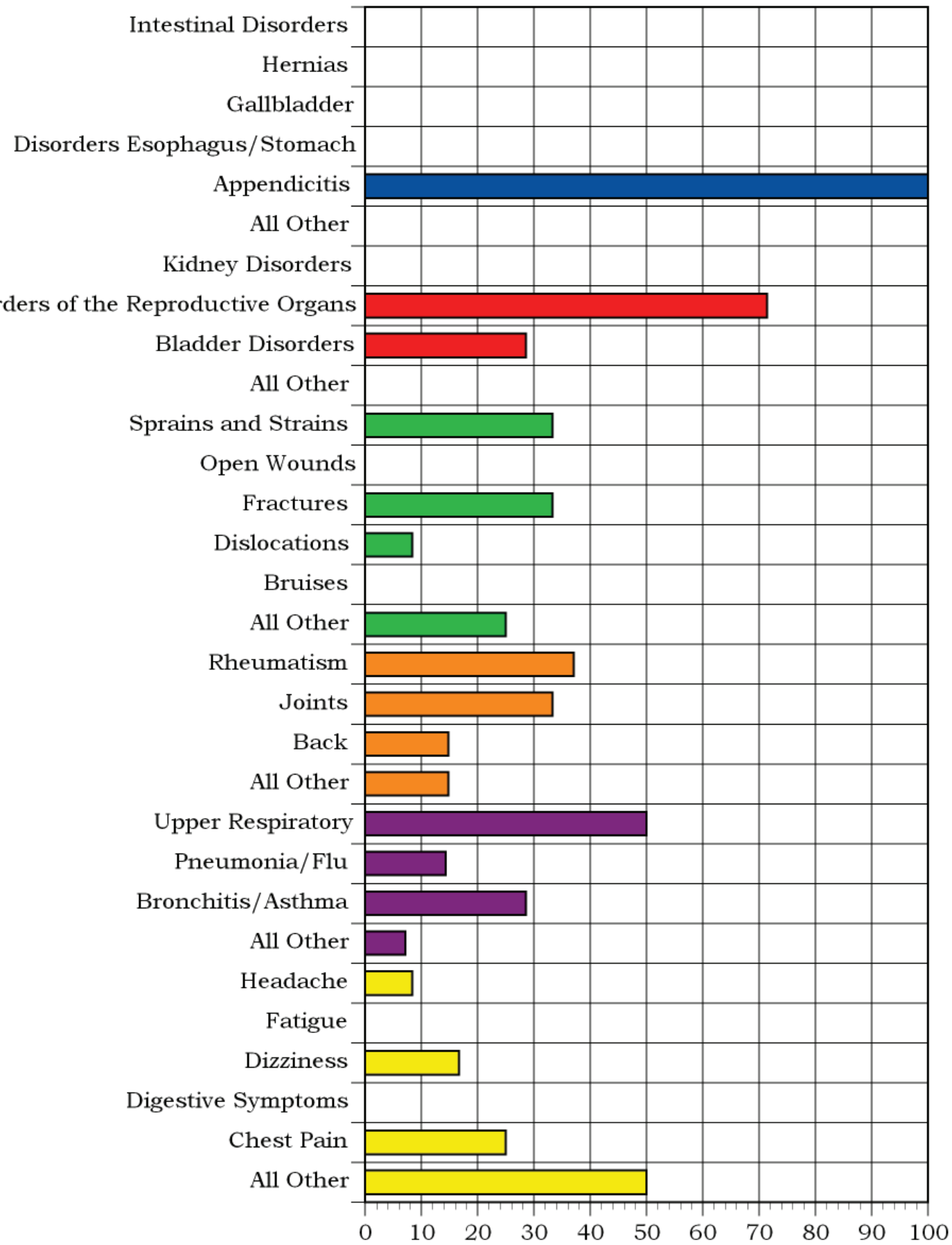

Percent Distribution of Diagnoses Within Diagnostic Category

Digestive, 1 Diagnoses

Genitourinary, 7 Diagnoses

Injury, 12 Diagnoses
Musculoskeletal, 27 Diagnoses

Respiratory, 14 Diagnoses

Unspecified Symptoms, 12 Diagnoses 
Figure 9. Common Diagnoses Among Male Workers in 2007

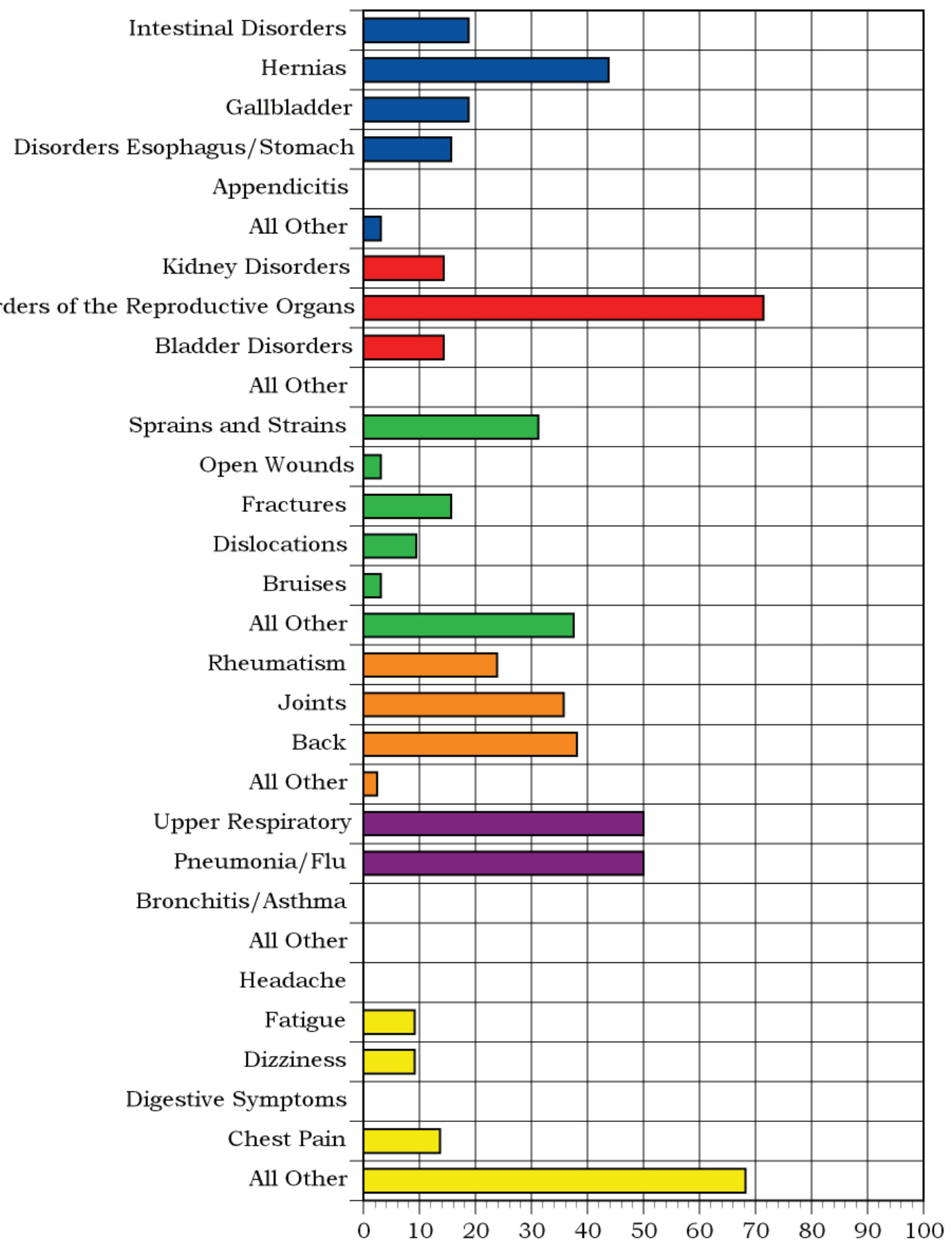

Percent Distribution of Diagnoses Within Diagnostic Category

Digestive, 32 Diagnoses

Genitourinary, 7 Diagnoses

Injury, 32 Diagnoses
Musculoskeletal, 42 Diagnoses

Respiratory, 6 Diagnoses

Unspecified Symptoms, 22 Diagnoses 


\section{Figure 10. Number of Most Frequently Reported Diagnoses by Job Category and Gender}

\begin{tabular}{|c|c|c|c|c|}
\hline Job Category & \multicolumn{2}{|l|}{ Men } & \multicolumn{2}{|l|}{ Women } \\
\hline \multirow{4}{*}{ Professional } & Musculoskeletal & 17 & Heart/Circulatory & 2 \\
\hline & Injury & 11 & Endocrine/Metabolic & 1 \\
\hline & Unspecified Symptoms & 9 & Musculoskeletal & 1 \\
\hline & & & Skin & 1 \\
\hline \multirow{4}{*}{ Administrative Support } & Digestive & 10 & Musculoskeletal & 16 \\
\hline & Musculoskeletal & 5 & Unspecified Symptoms & 6 \\
\hline & Injury & 2 & Genitourinary & 4 \\
\hline & & & Respiratory & 4 \\
\hline \multirow{4}{*}{ Technical Support } & Digestive & 9 & Musculoskeletal & 4 \\
\hline & Injury & 7 & Unspecified Symptoms & 3 \\
\hline & Heart/Circulatory & 6 & Heart/Circulatory & 2 \\
\hline & Musculoskeletal & 6 & & \\
\hline \multirow{3}{*}{ Service } & Musculoskeletal & 3 & Injury & 3 \\
\hline & Nervous System & 2 & Musculoskeletal & 2 \\
\hline & Unspecified Symptoms & 2 & Respiratory & 2 \\
\hline \multirow{3}{*}{ Security and Fire } & Musculoskeletal & 6 & Injury & 3 \\
\hline & Genitourinary & 2 & Respiratory & 2 \\
\hline & Unspecified Symptoms & 2 & Endocrine/Metabolic & 1 \\
\hline \multirow{3}{*}{ Crafts } & Injury & 5 & & 0 \\
\hline & Digestive & 4 & & \\
\hline & Musculoskeletal & 3 & & \\
\hline \multirow{3}{*}{ Line Operators } & Injury & 5 & Respiratory & 5 \\
\hline & Digestive & 3 & Injury & 4 \\
\hline & Musculoskeletal & 2 & Musculoskeletal & 4 \\
\hline
\end{tabular}




\section{Rates of Disease Occurrence}

Figure 11. Rates for All Illnesses and Injuries Combined by Job Category, Gender, and Age

\begin{tabular}{|c|c|c|c|c|}
\hline \multirow{2}{*}{$\begin{array}{c}\text { All Illnesses \& } \\
\text { Injuries Combined }\end{array}$} & \multicolumn{4}{|c|}{ Rate per 1,000} \\
\hline & Job Category & Age & Men & Women \\
\hline & \multirow{2}{*}{ Professional } & $<50$ & 77 & 28 \\
\hline & & $50+$ & 80 & 59 \\
\hline & \multirow{2}{*}{ Administrative Support } & $<50$ & 49 & 164 \\
\hline & & $50+$ & 110 & 171 \\
\hline & \multirow{2}{*}{ Technical Support } & $<50$ & 103 & 300 \\
\hline & & $50+$ & 157 & 163 \\
\hline & \multirow{2}{*}{ Service } & $<50$ & 111 & 0 \\
\hline & & $50+$ & 294 & 233 \\
\hline & \multirow{2}{*}{ Security and Fire } & $<50$ & 71 & 0 \\
\hline & & $50+$ & 289 & 1,200 \\
\hline & \multirow{2}{*}{ Crafts } & $<50$ & 49 & 0 \\
\hline & & $50+$ & 68 & 0 \\
\hline & \multirow{2}{*}{ Line Operators } & $<50$ & 119 & 86 \\
\hline & & $50+$ & 140 & 288 \\
\hline
\end{tabular}

Figure 12. Rates for Selected Diagnostic Categories by Job Category, Gender, and Age

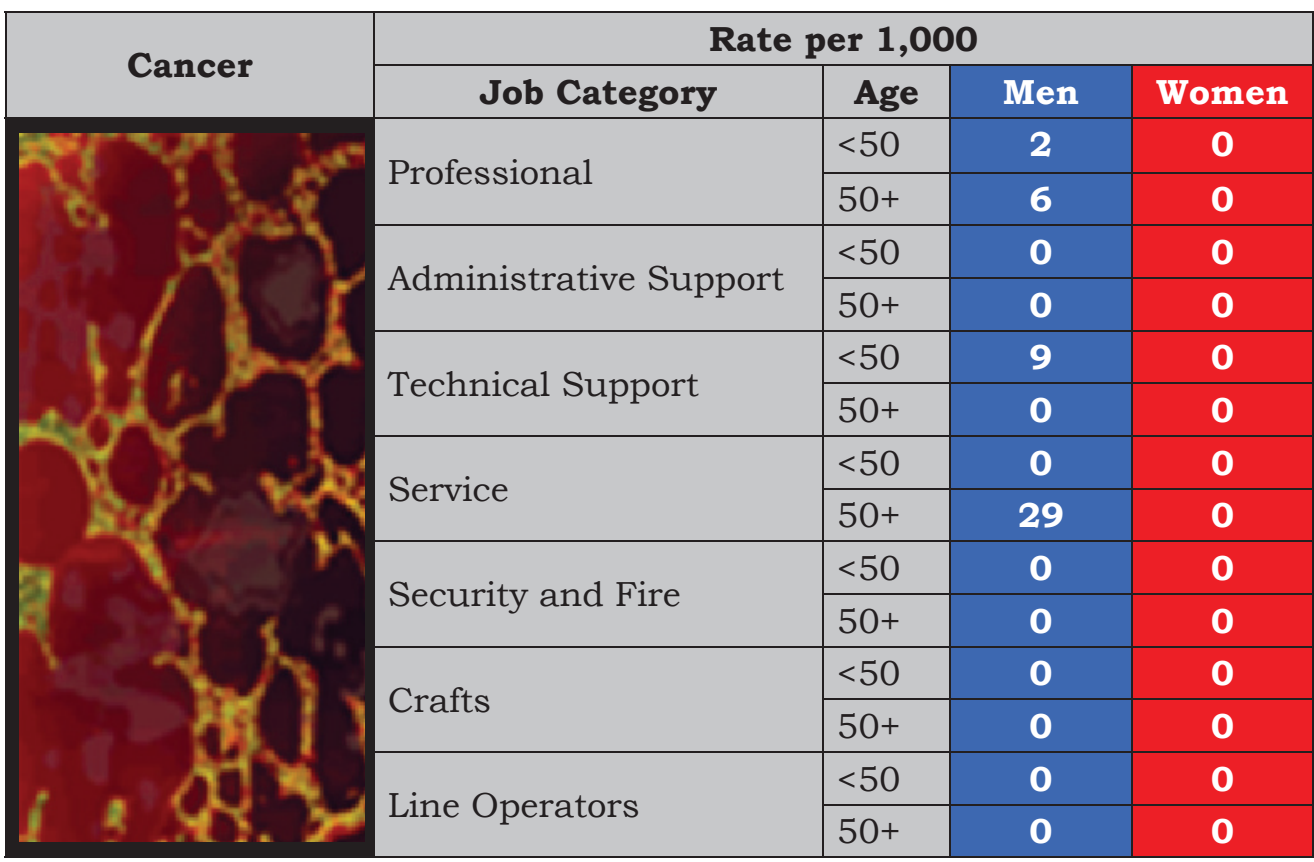




\section{Figure 12. Rates for Selected Diagnostic Categories by Job Category, Gender, and Age (Continued)}

\begin{tabular}{|c|c|c|c|c|}
\hline \multirow{2}{*}{ Heart/Circulatory } & \multicolumn{4}{|c|}{ Rate per 1,000} \\
\hline & Job Category & Age & Men & Women \\
\hline & \multirow{2}{*}{ Professional } & $<50$ & 4 & 9 \\
\hline & & $50+$ & 14 & 29 \\
\hline & \multirow{2}{*}{ Administrative Support } & $<50$ & 0 & 8 \\
\hline & & $50+$ & 0 & 14 \\
\hline & \multirow{2}{*}{ Technical Support } & $<50$ & 9 & 100 \\
\hline & & $50+$ & 29 & 0 \\
\hline & \multirow{2}{*}{ Service } & $<50$ & 37 & 0 \\
\hline & & $50+$ & 0 & 0 \\
\hline & \multirow{2}{*}{ Security and Fire } & $<50$ & 0 & 0 \\
\hline & & $50+$ & 26 & 0 \\
\hline & \multirow{2}{*}{ Crafts } & $<50$ & 0 & 0 \\
\hline & & $50+$ & 5 & 0 \\
\hline & \multirow{2}{*}{ Line Operators } & $<50$ & 0 & 0 \\
\hline & & $50+$ & 18 & 0 \\
\hline
\end{tabular}

\begin{tabular}{|c|c|c|c|c|}
\hline \multirow{2}{*}{ Respiratory } & \multicolumn{4}{|c|}{ Rate per 1,000} \\
\hline & Job Category & Age & Men & Women \\
\hline & \multirow{2}{*}{ Professional } & $<50$ & 2 & 0 \\
\hline & & $50+$ & 0 & 0 \\
\hline & \multirow{2}{*}{ Administrative Support } & $<50$ & 7 & 25 \\
\hline & & $50+$ & 0 & 7 \\
\hline & \multirow{2}{*}{ Technical Support } & $<50$ & 0 & 0 \\
\hline & & $50+$ & 0 & 23 \\
\hline & \multirow{2}{*}{ Service } & $<50$ & 0 & 0 \\
\hline & & $50+$ & 0 & 67 \\
\hline & \multirow{2}{*}{ Security and Fire } & $<50$ & 0 & 0 \\
\hline & & $50+$ & 0 & 400 \\
\hline & \multirow{2}{*}{ Crafts } & $<50$ & 8 & 0 \\
\hline & & $50+$ & 5 & 0 \\
\hline & \multirow{2}{*}{ Line Operators } & $<50$ & 30 & 0 \\
\hline & & $50+$ & 0 & 68 \\
\hline
\end{tabular}


Figure 12. Rates for Selected Diagnostic Categories by Job Category, Gender, and Age (Continued)

\begin{tabular}{|c|c|c|c|c|}
\hline \multirow{2}{*}{ Injury } & \multicolumn{4}{|c|}{ Rate per 1,000} \\
\hline & Job Category & Age & Men & Women \\
\hline \multirow{14}{*}{18} & \multirow{2}{*}{ Professional } & $<50$ & 13 & 0 \\
\hline & & $50+$ & 14 & 0 \\
\hline & \multirow{2}{*}{ Administrative Support } & $<50$ & 7 & 8 \\
\hline & & $50+$ & 8 & 0 \\
\hline & \multirow{2}{*}{ Technical Support } & $<50$ & 9 & 0 \\
\hline & & $50+$ & 35 & 23 \\
\hline & \multirow{2}{*}{ Service } & $<50$ & 0 & 0 \\
\hline & & $50+$ & 29 & 100 \\
\hline & \multirow{2}{*}{ Security and Fire } & $<50$ & 36 & 0 \\
\hline & & $50+$ & 0 & 600 \\
\hline & \multirow{2}{*}{ Crafts } & $<50$ & 8 & 0 \\
\hline & & $50+$ & 18 & 0 \\
\hline & \multirow{2}{*}{ Line Operators } & $<50$ & 45 & 29 \\
\hline & & $50+$ & 35 & 41 \\
\hline
\end{tabular}

\section{Time Trends}

Figure 13. Age-Adjusted Rates for All Diagnoses Combined Among Women and Men from 2003 to 2007*

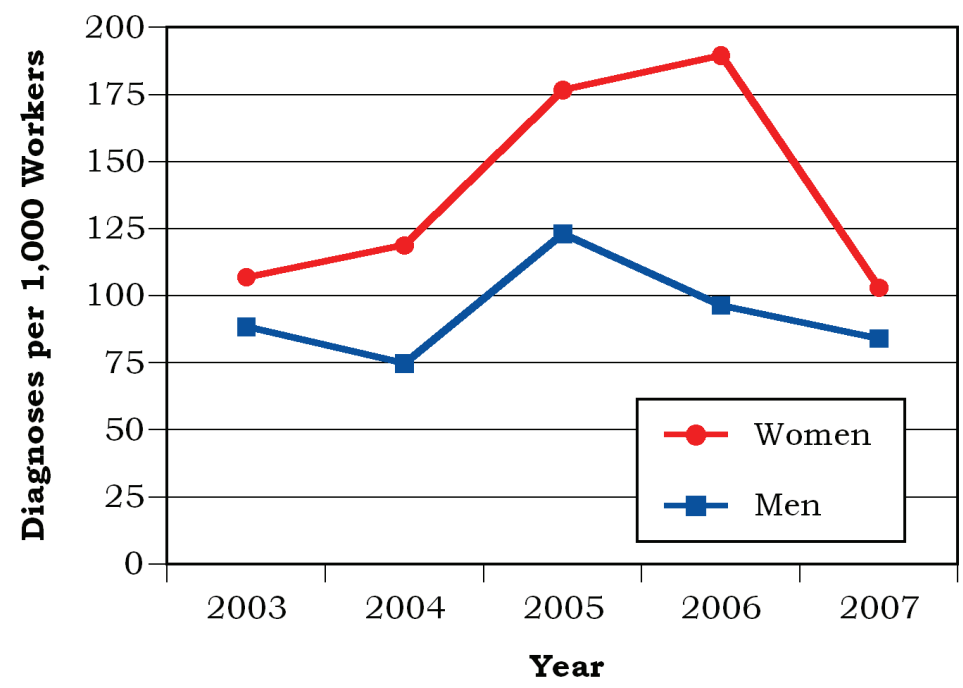

* Standardized to age distribution of 2000 U.S. population. 
Figure 14. Age-Adjusted Rates for Selected Diagnostic Categories Among Women and Men from 2003 to 2007*

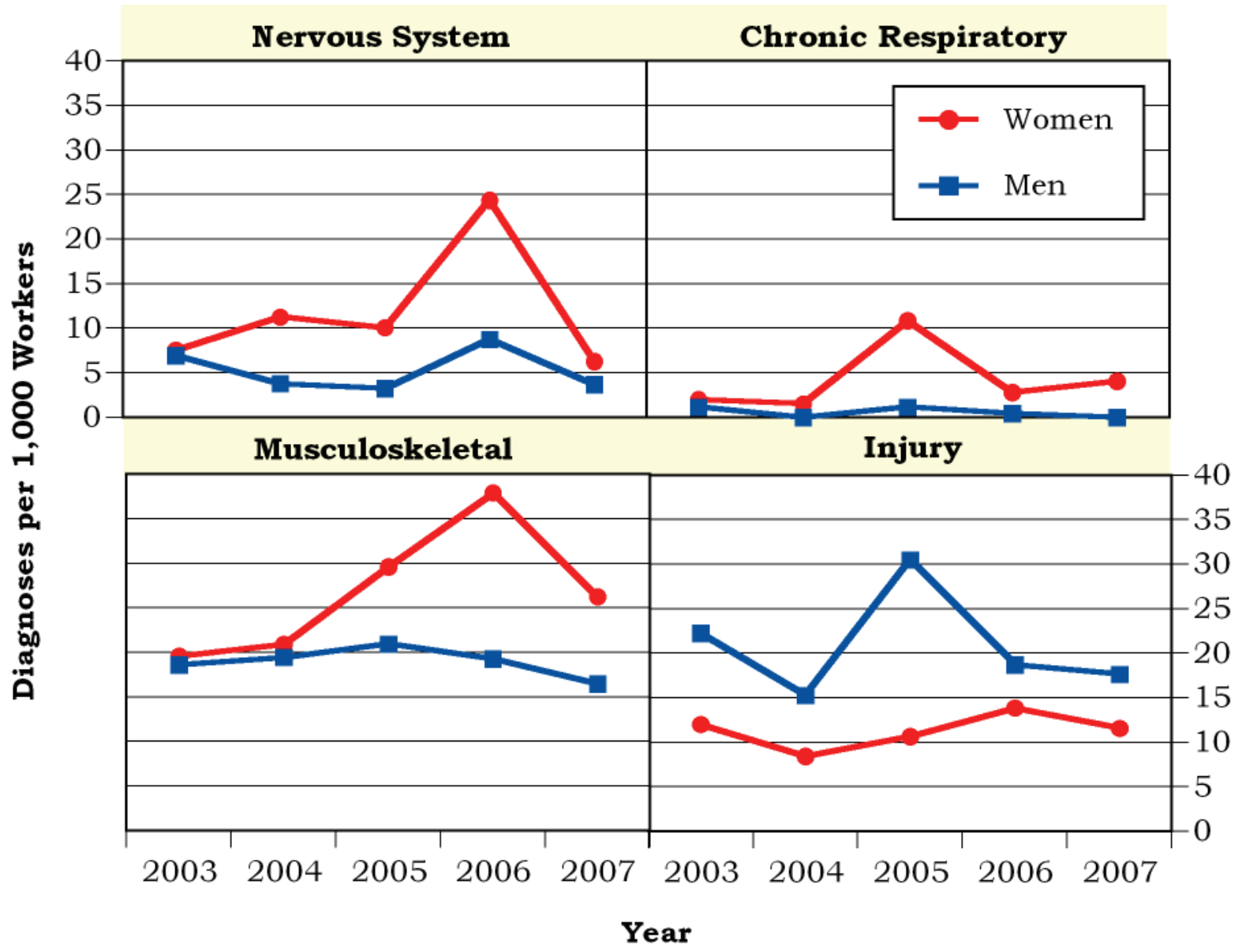

* Standardized to age distribution of 2000 U.S. population. 
Figure 15. Age-Adjusted Rates for All Diagnoses Combined Among Women and Men by Job Category from 2003 to 2007*

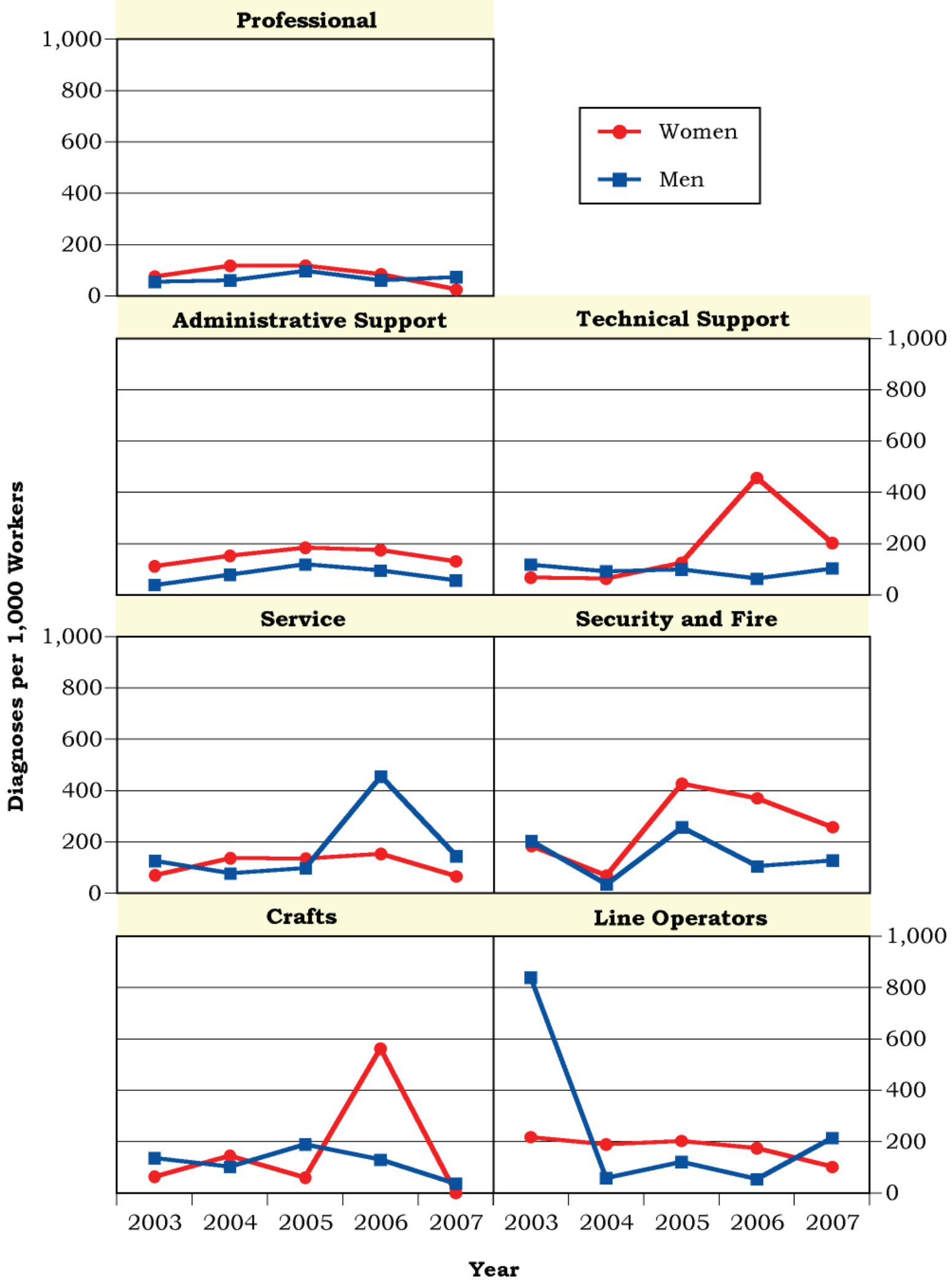

* Standardized to age distribution of 2000 U.S. population. 


\section{Sentinel Health Events for Occupations (SHEOs)}

An occupational sentinel health event (SHEO) is a disease, disability, or death that is likely to be occupationally related. Although sentinel health events may indicate an occupational exposure, many may result from nonoccupational exposures. Sentinel health events are therefore assessed in two categories:

Definite Sentinel Health Events: Diseases that are unlikely to occur in the absence of an occupational exposure (e.g., asbestosis).

Possible Sentinel Health Events: Diseases that may be occupational but can also occur in the absence of an occupational exposure (e.g., lung cancer or carpal tunnel syndrome).

Figure 16. Characteristics of SHEOs by Gender

\begin{tabular}{|l|c|c|c|c|}
\hline \multirow{2}{*}{} & \multicolumn{2}{|c|}{$\begin{array}{c}\text { Total Number of } \\
\text { SHEO Diagnoses }\end{array}$} & \multicolumn{2}{c|}{$\begin{array}{c}\text { Total Number of } \\
\text { Days Absent }\end{array}$} \\
\cline { 2 - 5 } & Men & Women & Men & Women \\
\hline Definite & 1 & 0 & 84 & 0 \\
\hline Possible & 4 & 5 & 27 & 349 \\
\hline Total & 5 & 5 & 111 & 349 \\
\hline
\end{tabular}

Figure 17. SHEO Diagnoses by Gender

\begin{tabular}{|l|c|c|}
\hline \multirow{2}{*}{\multicolumn{1}{|c|}{ Diagnoses }} & \multicolumn{2}{c|}{ Gender } \\
\cline { 2 - 3 } & Women & Men \\
\hline Carpal Tunnel Syndrome & 4 & 4 \\
\hline Pneumonconiosis (Berylliosis) & 0 & 0 \\
\hline Musculoskeletal Conditions & 0 & 0 \\
\hline Injuries & 0 & 1 \\
\hline Other Conditions & 1 & 0 \\
\hline
\end{tabular}


Occupational Safety and Health Administration (OSHA)-Recordable Events

Figure 18. OSHA-Recordable Events by Gender and Age

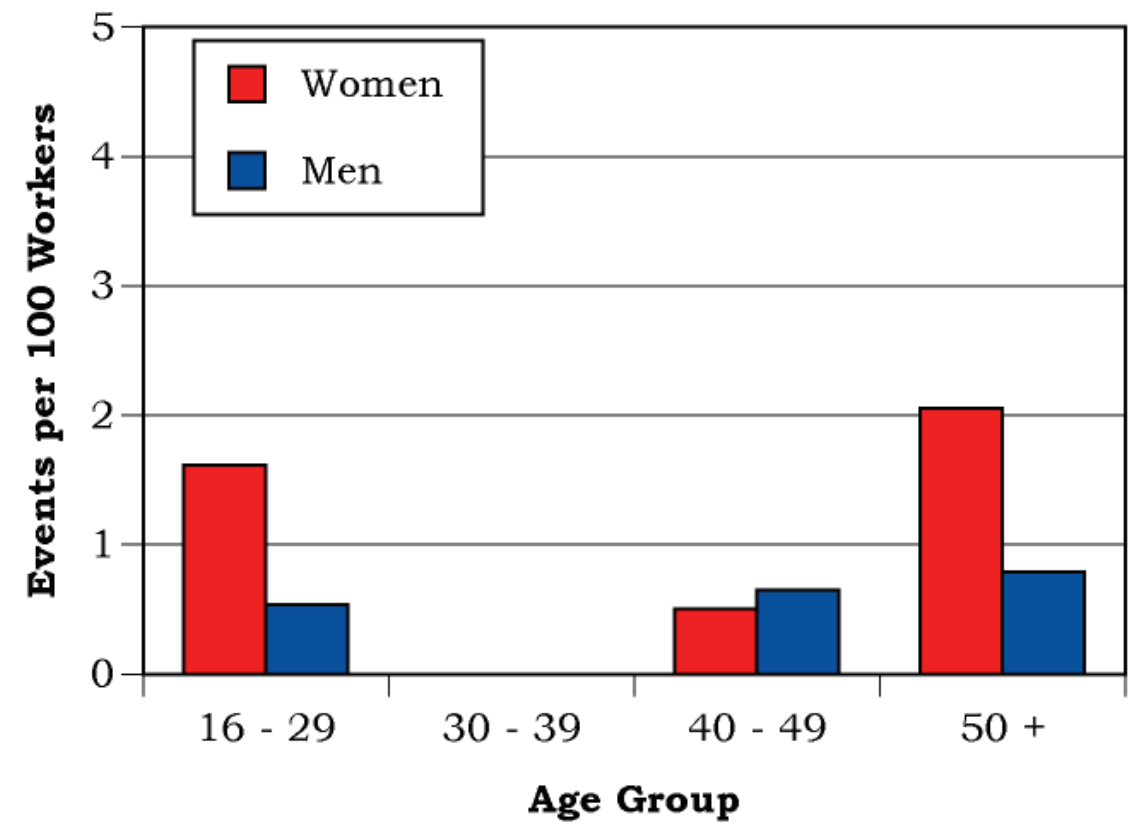

Figure 19. OSHA-Recordable Events by Job Category and Gender

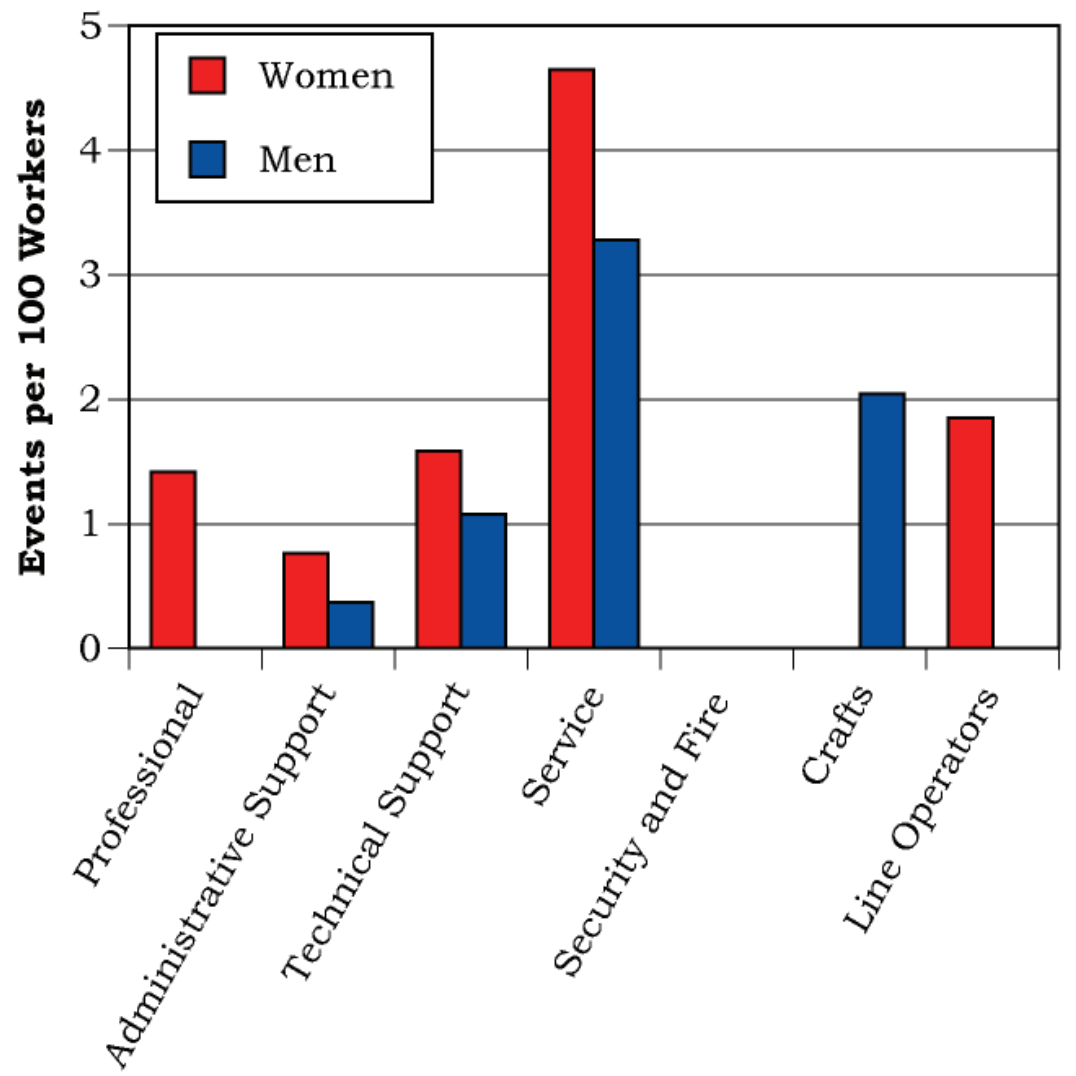

Job Category 


\section{Diagnostic and Accident Categories for OSHA-Recordable Events}

Figure 20. OSHA-Recordable Diagnoses by Diagnostic Category and Gender

\begin{tabular}{|l|c|c|}
\hline \multirow{2}{*}{ Diagnostic Category } & \multicolumn{2}{|c|}{ Gender } \\
\cline { 2 - 3 } & Women & Men \\
\hline Musculoskeletal & $\mathbf{4}$ & $\mathbf{1 4}$ \\
\hline Nervous System & $\mathbf{1}$ & $\mathbf{0}$ \\
\hline Respiratory & $\mathbf{1}$ & $\mathbf{0}$ \\
\hline Unspecified Symptoms & $\mathbf{0}$ & $\mathbf{2}$ \\
\hline Injury & $\mathbf{1 2}$ & $\mathbf{9}$ \\
\hline Fractures - Upper Limb & $\mathbf{1}$ & $\mathbf{1}$ \\
\hline Fractures - Lower Limb & $\mathbf{2}$ & $\mathbf{0}$ \\
\hline Dislocations & $\mathbf{1}$ & $\mathbf{0}$ \\
\hline Back Sprains \& Strains & $\mathbf{1}$ & $\mathbf{0}$ \\
\hline Other Sprains \& Strains & $\mathbf{5}$ & $\mathbf{3}$ \\
\hline Open Wounds - Upper Limb & $\mathbf{0}$ & $\mathbf{4}$ \\
\hline Superficial Injuries & $\mathbf{1}$ & $\mathbf{0}$ \\
\hline Foreign Bodies Entering Orifice & $\mathbf{0}$ & $\mathbf{1}$ \\
\hline Unspecified Injuries & $\mathbf{1}$ & $\mathbf{0}$ \\
\hline
\end{tabular}

Figure 21. OSHA-Recordable Accidents by Type and Gender

\begin{tabular}{|l|c|c|}
\hline \multirow{2}{*}{\multicolumn{1}{|c|}{ Accident Category }} & \multicolumn{2}{c|}{ Gender } \\
\cline { 2 - 3 } & Women & Men \\
\cline { 2 - 3 } & $\begin{array}{c}\text { Number of } \\
\text { Accidents }\end{array}$ & $\begin{array}{c}\text { Number of } \\
\text { Accidents }\end{array}$ \\
\hline Motor Vehicle Non-Traffic & $\mathbf{1}$ & $\mathbf{0}$ \\
\hline Falls & $\mathbf{3}$ & $\mathbf{4}$ \\
\hline Natural/Environmental Factors & $\mathbf{1}$ & $\mathbf{0}$ \\
\hline Submersion/Suffocation/Foreign Bodies & $\mathbf{0}$ & $\mathbf{1}$ \\
\hline Other Accidents & $\mathbf{3}$ & $\mathbf{8}$ \\
\hline Struck by an Object & $\mathbf{0}$ & $\mathbf{2}$ \\
\hline Caught Between Objects & $\mathbf{0}$ & $\mathbf{1}$ \\
\hline Cutting/Piercing Instrument/Object & $\mathbf{0}$ & $\mathbf{2}$ \\
\hline Overexertion/Strenuous Movements & $\mathbf{2}$ & $\mathbf{3}$ \\
\hline Repetitive Trauma & $\mathbf{1}$ & $\mathbf{0}$ \\
\hline Total & $\mathbf{8}$ & $\mathbf{1 3}$ \\
\hline
\end{tabular}




\section{Rates of OSHA-Recordable Events}

Figure 22. OSHA-Recordable Rates by Age and Job Categories Among Women, All Diagnoses Combined

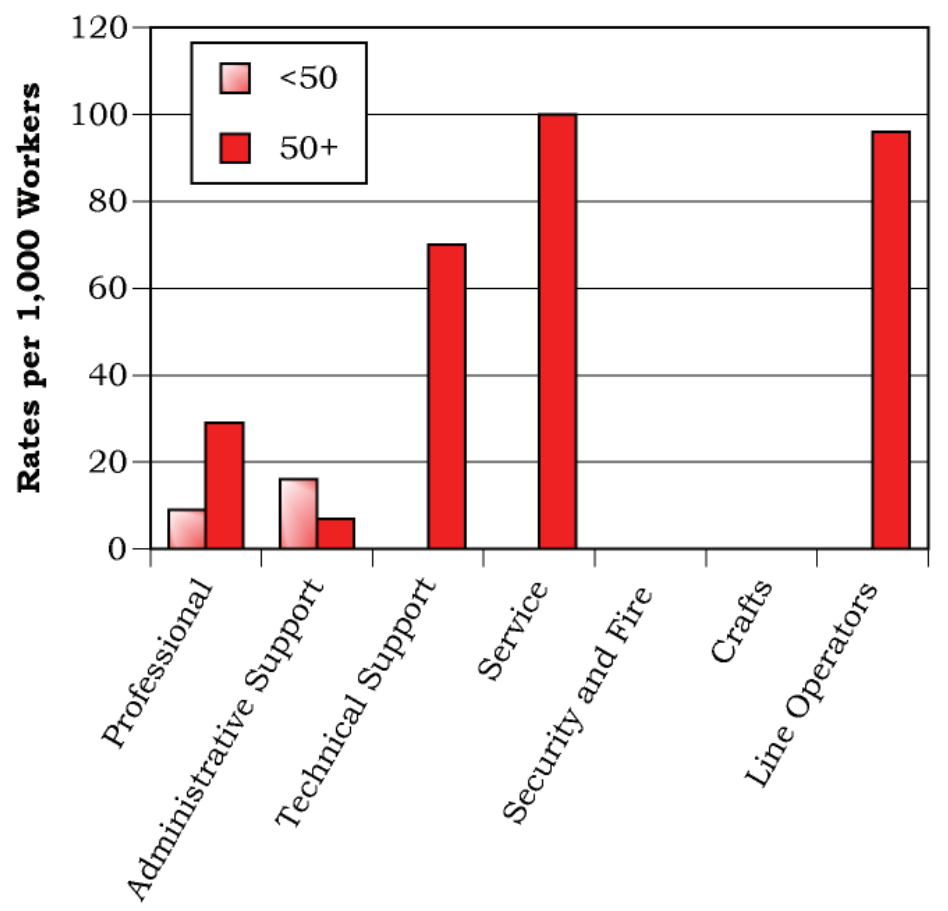

Job Category

Figure 23. OSHA-Recordable Rates by Age and Job Categories Among Men, All Diagnoses Combined

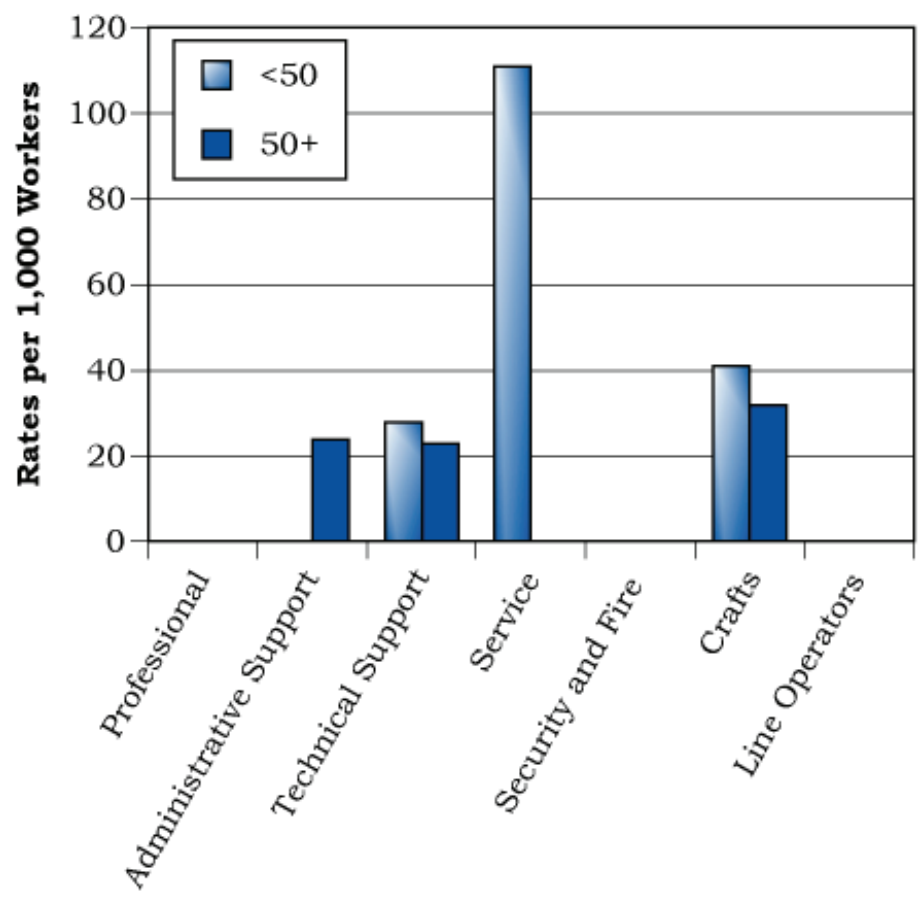




\section{Time Trends for OSHA-Recordable Events}

Figure 24. Age-Adjusted Rates for All OSHA-Recordable Diagnoses Combined Among Women and Men by Job Category from 2003 to 2007*

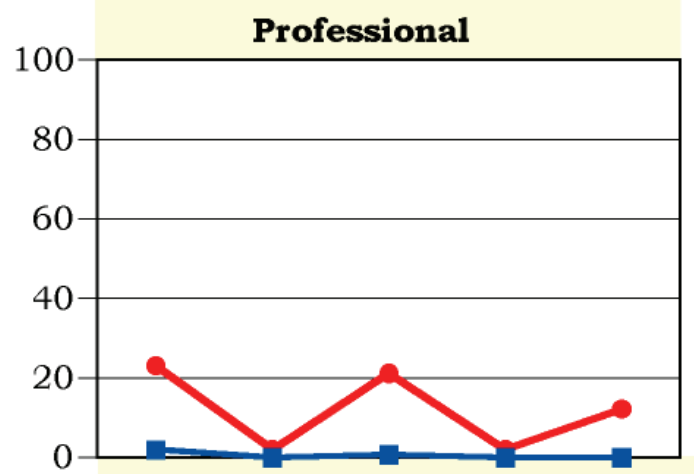

$$
\begin{aligned}
& - \text { Women } \\
& - \text { Men }
\end{aligned}
$$

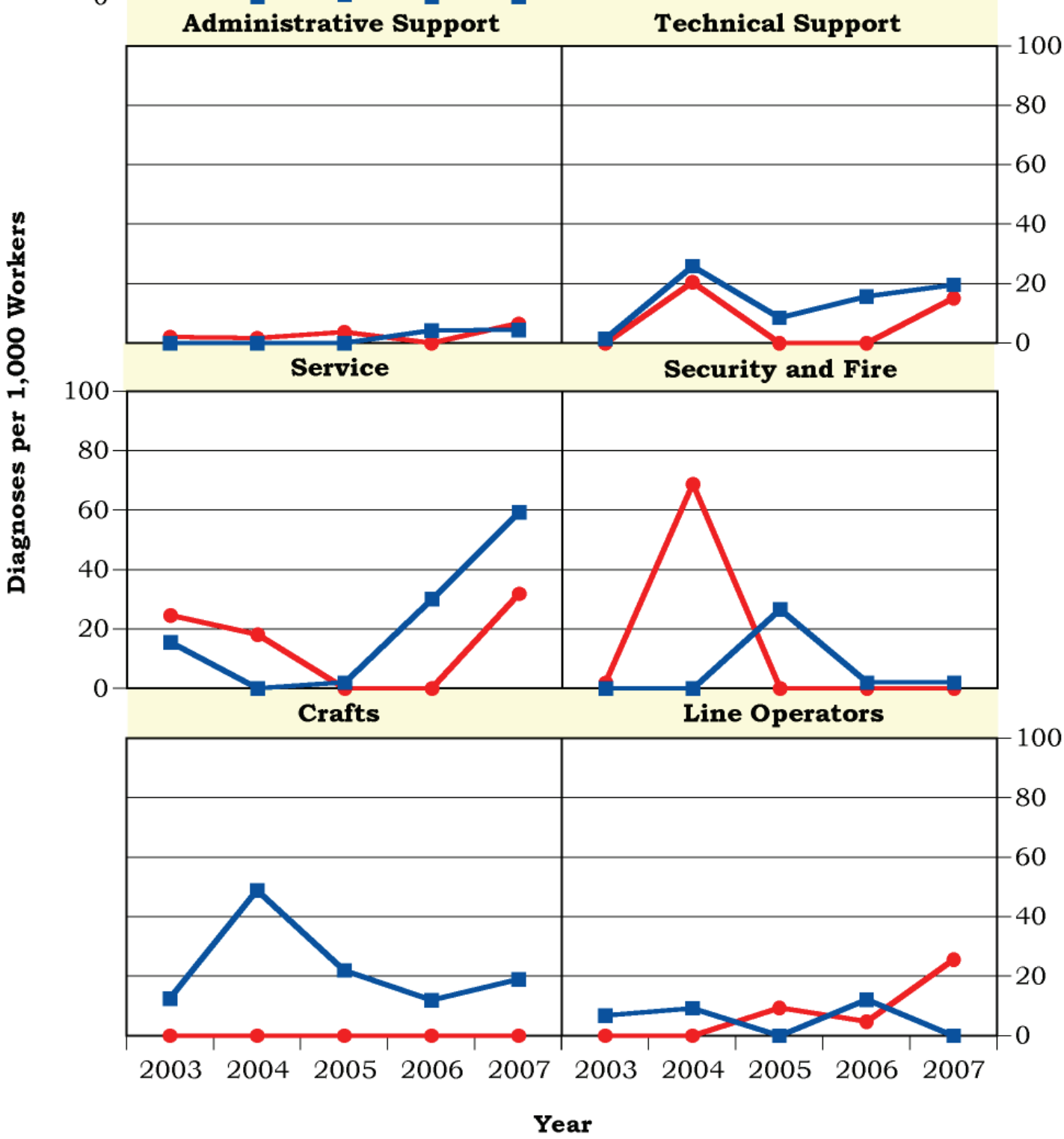

* Standardized to age distribution of 2000 U.S. population. 


\section{Appendices}


Kansas City Plant 2007

Absence Data

Appendix A. Work Force by Gender, Age, and Job Category

\begin{tabular}{|c|c|c|c|c|c|c|c|c|c|c|c|}
\hline \multirow{3}{*}{ Job Category } & \multicolumn{5}{|c|}{ Women } & \multicolumn{5}{|c|}{ Men } & \multirow[b]{3}{*}{ TOTAL } \\
\hline & \multicolumn{4}{|c|}{ Age Group } & \multirow[b]{2}{*}{ TOTAL } & \multicolumn{4}{|c|}{ Age Group } & \multirow[b]{2}{*}{ TOTAL } & \\
\hline & $16-29$ & $30-39$ & $40-49$ & $50+$ & & $16-29$ & 30 - 39 & $40-49$ & $50+$ & & \\
\hline Professional & 36 & 21 & 50 & 34 & 141 & 95 & 77 & 298 & 362 & 832 & 973 \\
\hline Administrative Support & 14 & 17 & 91 & 140 & 262 & 28 & 28 & 86 & 127 & 269 & 531 \\
\hline Technical Support & 1 & 4 & 15 & 43 & 63 & 20 & 20 & 67 & 172 & 279 & 342 \\
\hline Service & 6 & 0 & 7 & 30 & 43 & 15 & 1 & 11 & 34 & 61 & 104 \\
\hline Security and Fire & 2 & 2 & 3 & 5 & 12 & 6 & 9 & 13 & 38 & 66 & 78 \\
\hline Crafts & 0 & 0 & 3 & 16 & 19 & 5 & 20 & 98 & 219 & 342 & 361 \\
\hline Line Operators & 3 & 2 & 30 & 73 & 108 & 17 & 9 & 41 & 57 & 124 & 232 \\
\hline TOTAL & 62 & 46 & 199 & 341 & 648 & 186 & 164 & 614 & 1,009 & 1,973 & 2,621 \\
\hline
\end{tabular}

Appendix B. Age Distribution of the Work Force by Gender

\begin{tabular}{|l|r|r|r|r|r|r|r|r|}
\hline \multirow{3}{*}{ Year } & \multicolumn{4}{|c|}{ Women } & \multicolumn{4}{c|}{ Men } \\
\cline { 2 - 9 } & \multicolumn{2}{|c|}{ Percent Distribution by Age Group } & \multicolumn{1}{c|}{ Percent Distribution by Age Group } \\
\cline { 2 - 9 } & $\mathbf{1 6}-\mathbf{2 9}$ & $\mathbf{3 0}-\mathbf{3 9}$ & $\mathbf{4 0 - 4 9}$ & $\mathbf{5 0}+$ & $\mathbf{1 6}-\mathbf{2 9}$ & $\mathbf{3 0}-\mathbf{3 9}$ & $\mathbf{4 0}-\mathbf{4 9}$ & $\mathbf{5 0}+$ \\
\hline $\mathbf{2 0 0 2}$ & 9.74 & 9.62 & 40.68 & 39.95 & 8.47 & 8.60 & 42.40 & 40.53 \\
\hline $\mathbf{2 0 0 3}$ & 6.88 & 9.04 & 40.76 & 43.31 & 8.02 & 7.46 & 42.54 & 41.98 \\
\hline $\mathbf{2 0 0 4}$ & 10.52 & 7.79 & 37.98 & 43.72 & 9.04 & 7.25 & 40.96 & 42.75 \\
\hline $\mathbf{2 0 0 5}$ & 9.86 & 7.46 & 35.63 & 47.04 & 8.51 & 7.66 & 37.94 & 45.88 \\
\hline $\mathbf{2 0 0 6}$ & 9.23 & 6.85 & 33.78 & 50.15 & 8.31 & 7.81 & 34.78 & 49.10 \\
\hline $\mathbf{2 0 0 7}$ & 9.57 & 7.10 & 30.71 & 52.62 & 9.43 & 8.31 & 31.12 & 51.14 \\
\hline
\end{tabular}


Kansas City Plant 2007

Absence Data

Appendix C. Total Number of Workers Who Reported at Least One Absence by Gender, Age, and Job Category*

\begin{tabular}{|c|c|c|c|c|c|c|c|c|c|c|c|}
\hline \multirow{3}{*}{ Job Category } & \multicolumn{5}{|c|}{ Women } & \multicolumn{5}{|c|}{ Men } & \multirow[b]{3}{*}{ TOTAL } \\
\hline & \multicolumn{4}{|c|}{ Age Group } & \multirow[b]{2}{*}{ TOTAL } & \multicolumn{4}{|c|}{ Age Group } & \multirow[b]{2}{*}{ TOTAL } & \\
\hline & $16-29$ & 30 - 39 & $40-49$ & $50+$ & & $16-29$ & 30 - 39 & $40-49$ & $50+$ & & \\
\hline Professional & 0 & 0 & 3 & 1 & 4 & 1 & 4 & 14 & 20 & 39 & 43 \\
\hline Administrative Support & 1 & 0 & 10 & 14 & 25 & 1 & 1 & 5 & 6 & 13 & 38 \\
\hline Technical Support & 0 & 1 & 1 & 4 & 6 & 0 & 1 & 6 & 15 & 22 & 28 \\
\hline Service & 0 & 0 & 0 & 5 & 5 & 0 & 0 & 2 & 5 & 7 & 12 \\
\hline Security and Fire & 0 & 0 & 0 & 2 & 2 & 0 & 1 & 1 & 4 & 6 & 8 \\
\hline Crafts & 0 & 0 & 0 & 0 & 0 & 0 & 0 & 4 & 10 & 14 & 14 \\
\hline Line Operators & 0 & 0 & 2 & 13 & 15 & 0 & 2 & 2 & 6 & 10 & 25 \\
\hline TOTAL & 1 & 1 & 16 & 39 & 57 & 2 & 9 & 34 & 66 & 111 & 168 \\
\hline
\end{tabular}

*Only those job categories and gender/age combinations with at least one absence appear in this table.

Appendix D. Total Number of Absences by Gender, Age, and Job Category*

\begin{tabular}{|c|c|c|c|c|c|c|c|c|c|c|c|}
\hline \multirow{3}{*}{ Job Category } & \multicolumn{5}{|c|}{ Women } & \multicolumn{5}{|c|}{ Men } & \multirow[b]{3}{*}{ TOTAL } \\
\hline & \multicolumn{4}{|c|}{ Age Group } & \multirow[b]{2}{*}{ TOTAL } & \multicolumn{4}{|c|}{ Age Group } & \multirow[b]{2}{*}{ TOTAL } & \\
\hline & $16-29$ & 30 - 39 & $40-49$ & $50+$ & & $16-29$ & 30 - 39 & $40-49$ & $50+$ & & \\
\hline Professional & 0 & 0 & 3 & 1 & 4 & 1 & 4 & 14 & 23 & 42 & 46 \\
\hline Administrative Support & 1 & 0 & 10 & 15 & 26 & 1 & 1 & 5 & 7 & 14 & 40 \\
\hline Technical Support & 0 & 1 & 1 & 4 & 6 & 0 & 1 & 6 & 16 & 23 & 29 \\
\hline Service & 0 & 0 & 0 & 6 & 6 & 0 & 0 & 2 & 6 & 8 & 14 \\
\hline Security and Fire & 0 & 0 & 0 & 4 & 4 & 0 & 1 & 1 & 4 & 6 & 10 \\
\hline Crafts & 0 & 0 & 0 & 0 & 0 & 0 & 0 & 5 & 12 & 17 & 17 \\
\hline Line Operators & 0 & 0 & 3 & 13 & 16 & 0 & 2 & 2 & 6 & 10 & 26 \\
\hline TOTAL & 1 & 1 & 17 & 43 & 62 & 2 & 9 & 35 & 74 & 120 & 182 \\
\hline
\end{tabular}

*Only those job categories and gender/age combinations with at least one absence appear in this table. 
Kansas City Plant 2007

Absence Data

Appendix E. Distribution of the Number of Calendar Days Missed per Absence by Gender and Age*

\begin{tabular}{|c|c|c|c|c|c|c|c|c|c|c|c|}
\hline \multirow{3}{*}{$\begin{array}{c}\text { Number of } \\
\text { Calendar Days }\end{array}$} & \multicolumn{5}{|c|}{ Women } & \multicolumn{5}{|c|}{ Men } & \multirow[b]{3}{*}{ TOTAL } \\
\hline & \multicolumn{4}{|c|}{ Age Group } & \multirow[b]{2}{*}{ TOTAL } & \multicolumn{4}{|c|}{ Age Group } & \multirow[b]{2}{*}{ TOTAL } & \\
\hline & $16-29$ & $30-39$ & $40-49$ & $50+$ & & $16-29$ & 30 - 39 & $40-49$ & $50+$ & & \\
\hline$<15$ & 0 & 1 & 11 & 14 & 26 & 2 & 7 & 15 & 29 & 53 & 79 \\
\hline $15-28$ & 1 & 0 & 4 & 12 & 17 & 0 & 2 & 6 & 14 & 22 & 39 \\
\hline $29-42$ & 0 & 0 & 1 & 2 & 3 & 0 & 0 & 10 & 10 & 20 & 23 \\
\hline $43-56$ & 0 & 0 & 1 & 6 & 7 & 0 & 0 & 1 & 9 & 10 & 17 \\
\hline $57-91$ & 0 & 0 & 0 & 5 & 5 & 0 & 0 & 2 & 8 & 10 & 15 \\
\hline $92-182$ & 0 & 0 & 0 & 4 & 4 & 0 & 0 & 1 & 3 & 4 & 8 \\
\hline $183+$ & 0 & 0 & 0 & 0 & 0 & 0 & 0 & 0 & 1 & 1 & 1 \\
\hline TOTAL & 1 & 1 & 17 & 43 & 62 & 2 & 9 & 35 & 74 & 120 & 182 \\
\hline
\end{tabular}

*Only those gender/age combinations with at least one absence appear in this table. 
Kansas City Plant 2007

Absence Data

Appendix F. Distribution of the Number of Calendar Days Missed per Absence by Gender and Job Category*

Women

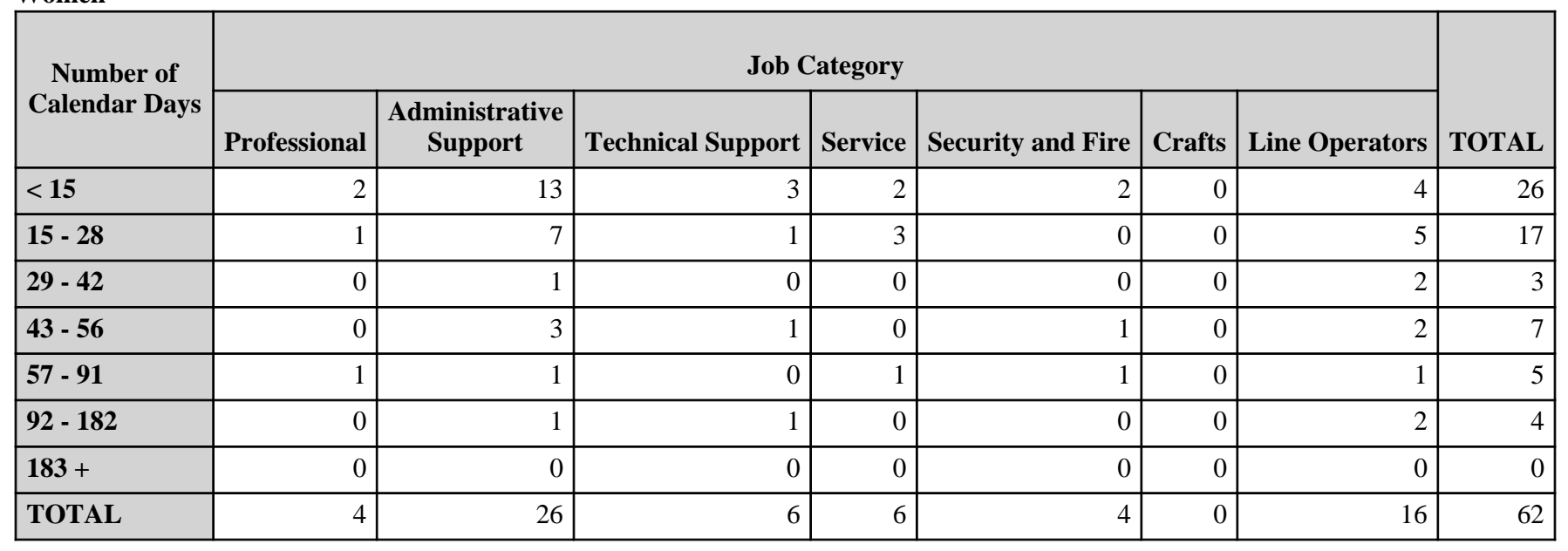

Men

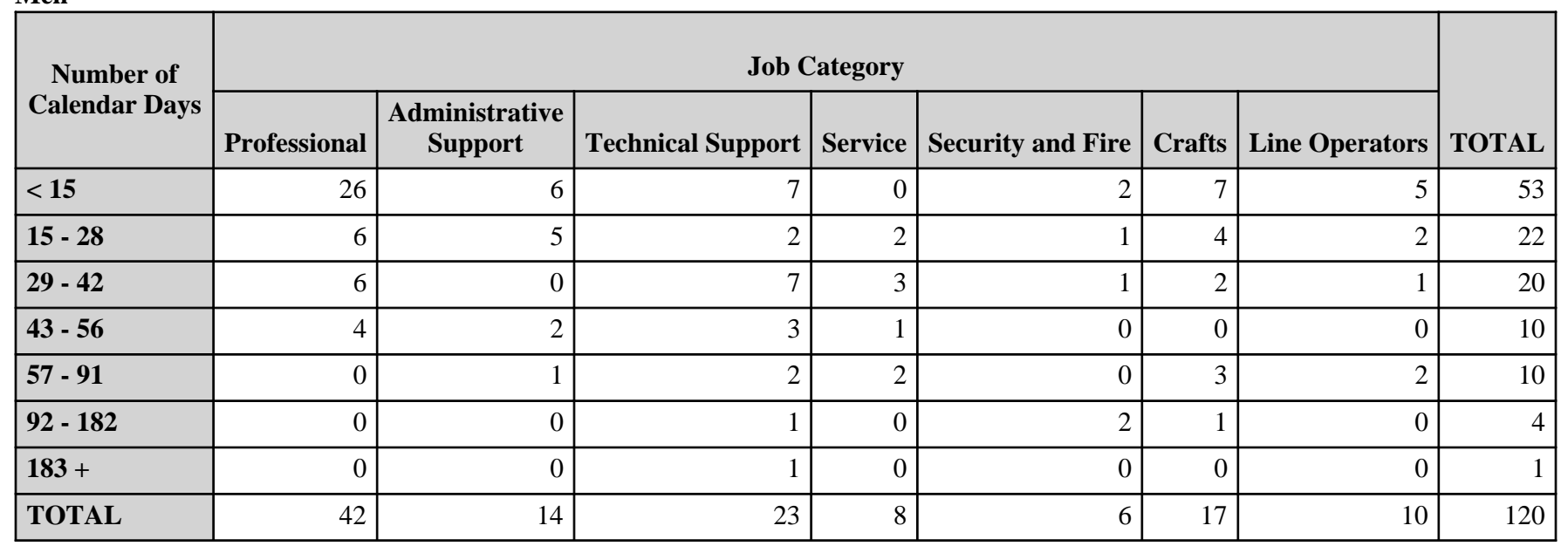

*Only those gender/job category combinations with at least one absence appear in this table. 


\section{Kansas City Plant 2007}

Absence Data

Appendix G. Number of Diagnoses in Each Diagnostic Category by Gender and Age*

\begin{tabular}{|c|c|c|c|c|c|c|}
\hline & & \multicolumn{5}{|c|}{ Women } \\
\hline & & \multicolumn{4}{|c|}{ Age Group } & \multirow[b]{2}{*}{ TOTAL } \\
\hline & & $16-29$ & $30-39$ & $40-49$ & $50+$ & \\
\hline Diagnostic Category & ICD-9-CM Code & & & & & \\
\hline INFECTIOUS \& PARASITIC DISEASES (DIS) & 001-139 & 0 & 0 & 0 & 1 & 1 \\
\hline -Other Bacterial Dis & $030-041$ & 0 & 0 & 0 & 0 & 0 \\
\hline -Viral Dis with Exanthem & 050-057 & 0 & 0 & 0 & 1 & 1 \\
\hline MALIGNANT NEOPLASMS & 140-208, 230-234 & 0 & 0 & 0 & 0 & 0 \\
\hline -Bone, Connective Tissue, Skin & $170-173,176$ & 0 & 0 & 0 & 0 & 0 \\
\hline -Genitourinary & 179-189 & 0 & 0 & 0 & 0 & 0 \\
\hline -Other \& Unspecified Sites & 190, 193-199 & 0 & 0 & 0 & 0 & 0 \\
\hline -Nervous System & 191-192 & 0 & 0 & 0 & 0 & $\overline{0}$ \\
\hline BENIGN \& UNCERTAIN NEOPLASMS & 210-229, 235-239 & 0 & 0 & 0 & 1 & 1 \\
\hline ENDOCRINE/METABOLIC/IMMUNITY & $240-279$ & 0 & 0 & 1 & 1 & 2 \\
\hline -Thyroid Gland Disorders & $240-246$ & 0 & 0 & 0 & 1 & 1 \\
\hline -Other Endocrine Gland Dis & $250-259$ & 0 & 0 & 0 & 0 & 0 \\
\hline -Other Metabolic \& Immunity Disorders & 270-279 & 0 & 0 & 1 & 0 & 1 \\
\hline BLOOD \& BLOOD-FORMING ORGANS & $280-289$ & 0 & 0 & 1 & 1 & 2 \\
\hline MENTAL DISORDERS & 290-319 & 0 & 0 & 1 & 0 & 1 \\
\hline -Non-Psychotic Disorders & $300-302,306-316$ & 0 & 0 & 1 & 0 & 1 \\
\hline NERVOUS SYSTEM (NS) \& SENSE ORGANS & $320-389$ & 0 & 0 & 1 & 5 & 6 \\
\hline -Other Disorders of Central NS & $340-349$ & 0 & 0 & 0 & 0 & 0 \\
\hline -Disorders of Peripheral NS & $350-359$ & 0 & 0 & 0 & 4 & 4 \\
\hline -Disorders of Eye & $360-379$ & 0 & 0 & 0 & 1 & 1 \\
\hline -Dis of Ear \& Mastoid & 380-389 & 0 & 0 & 1 & 0 & 1 \\
\hline CIRCULATORY SYSTEM & $390-459$ & 0 & 0 & 4 & 3 & 7 \\
\hline -Ischemic Heart Dis & $410-414$ & 0 & 0 & 0 & 1 & 1 \\
\hline -Dis of Pulmonary Circulation & $415-417$ & 0 & 0 & 1 & 0 & 1 \\
\hline -Other Heart Dis & $420-429$ & 0 & 0 & 1 & 1 & 2 \\
\hline -Cerebrovascular Dis & $430-438$ & 0 & 0 & 0 & 0 & 0 \\
\hline -Dis of Arteries \& Capillaries & $440-448$ & 0 & 0 & 0 & 0 & 0 \\
\hline -Dis of Veins, Lymphatics, Other & $451-459$ & 0 & 0 & 2 & 1 & 3 \\
\hline RESPIRATORY SYSTEM & $460-519$ & 1 & 0 & 2 & 11 & 14 \\
\hline -Acute Respiratory Infections & $460-466$ & 0 & 0 & 0 & 2 & 2 \\
\hline -Other Dis Upper Respiratory Tract & $470-478$ & 1 & 0 & 0 & 4 & 5 \\
\hline -Pneumonia \& Influenza & $480-487$ & 0 & 0 & 0 & 2 & 2 \\
\hline -Chronic Obstructive Dis & $490-496$ & 0 & 0 & 1 & 3 & 4 \\
\hline -Other Respiratory Dis & $510-519$ & 0 & 0 & 1 & 0 & 1 \\
\hline DIGESTIVE SYSTEM & $520-579$ & 0 & 0 & 1 & 0 & 1 \\
\hline -Esophagus, Stomach, Duodenum & $\mathbf{5 3 0 - 5 3 7}$ & 0 & 0 & 0 & 0 & 0 \\
\hline -Appendicitis & $540-543$ & 0 & 0 & 1 & 0 & 1 \\
\hline
\end{tabular}

*Only those diagnostic categories and gender/age combinations with at least one occurrence appear in this table. 


\section{Kansas City Plant 2007}

Absence Data

Appendix G. Number of Diagnoses in Each Diagnostic Category by Gender and Age*

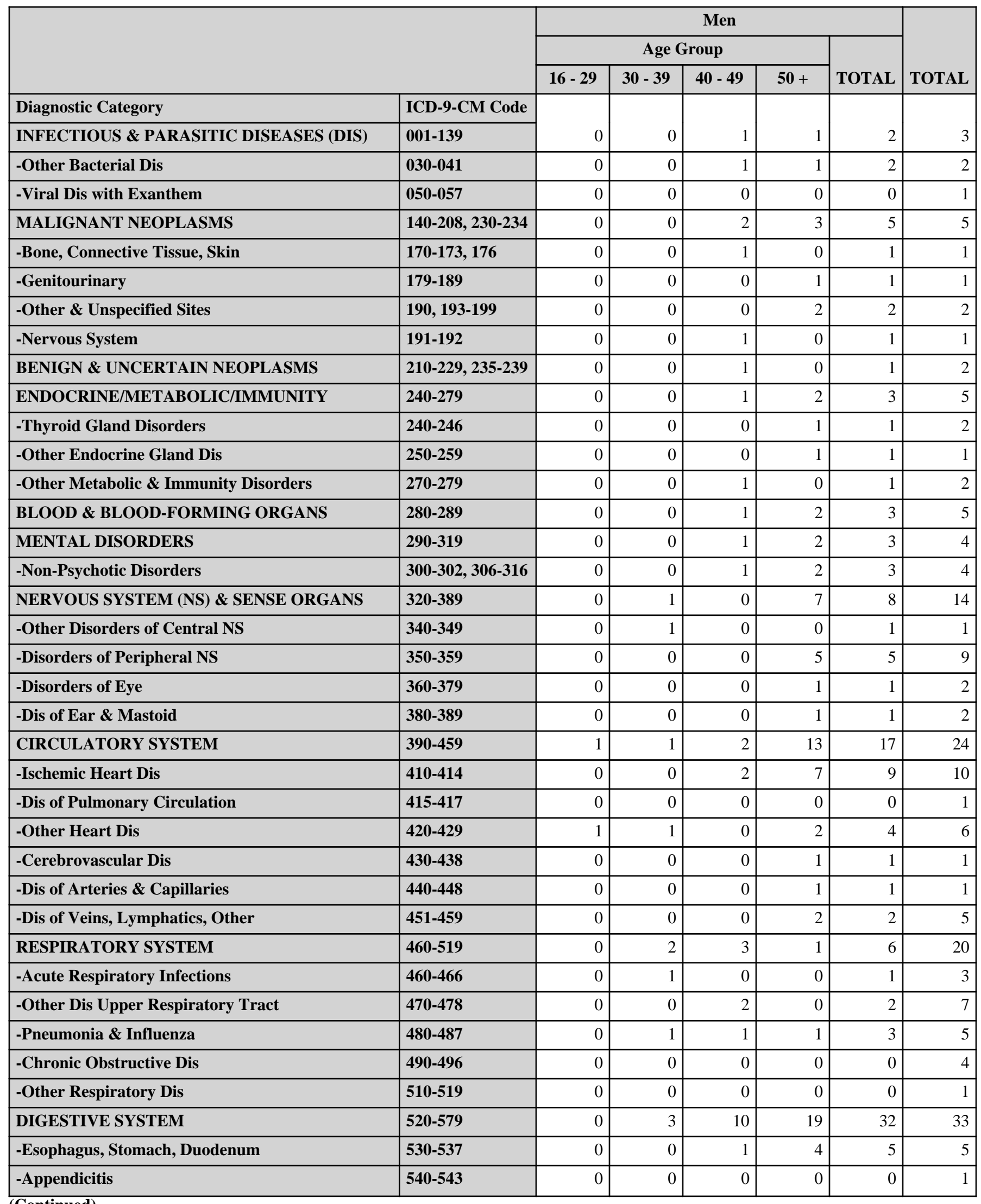

(Continued)

*Only those diagnostic categories and gender/age combinations with at least one occurrence appear in this table. 


\section{Kansas City Plant 2007}

Absence Data

Appendix G. Number of Diagnoses in Each Diagnostic Category by Gender and Age*

\begin{tabular}{|c|c|c|c|c|c|c|}
\hline & & \multicolumn{5}{|c|}{ Women } \\
\hline & & \multicolumn{4}{|c|}{ Age Group } & \multirow[b]{2}{*}{ TOTAL } \\
\hline & & $16-29$ & $30-39$ & $40-49$ & $50+$ & \\
\hline Diagnostic Category & ICD-9-CM Code & \multirow[b]{2}{*}{0} & \multirow[b]{2}{*}{0} & \multirow[b]{2}{*}{0} & \multirow[b]{2}{*}{0} & \multirow[b]{2}{*}{0} \\
\hline -Hernia & $550-553$ & & & & & \\
\hline -Enteritis, Colitis & $555-558$ & 0 & 0 & 0 & 0 & 0 \\
\hline -Other Intestinal Dis & $560-569$ & 0 & 0 & 0 & 0 & 0 \\
\hline -Other Digestive Dis & $570-579$ & 0 & 0 & 0 & 0 & 0 \\
\hline GENITOURINARY SYSTEM & $580-629$ & 0 & 0 & 2 & 5 & 7 \\
\hline -Other Urinary Dis & $590-599$ & 0 & 0 & 0 & 2 & 2 \\
\hline -Male Genital Organ Dis & $600-608$ & 0 & 0 & 0 & 0 & 0 \\
\hline -Other Female Disorders & $617-629$ & 0 & 0 & 2 & 3 & 5 \\
\hline SKIN \& SUBCUTANEOUS TISSUE & $680-709$ & 0 & 0 & 2 & 3 & 5 \\
\hline -Infections & $680-686$ & 0 & 0 & 0 & 2 & 2 \\
\hline -Other Inflammatory Conditions & $690-698$ & 0 & 0 & 2 & 0 & 2 \\
\hline -Other & 700-709 & 0 & 0 & 0 & 1 & 1 \\
\hline $\begin{array}{l}\text { MUSCULOSKELETAL \& CONNECTIVE } \\
\text { TISSUE }\end{array}$ & 710-739 & 0 & 0 & 8 & 19 & 27 \\
\hline -Arthropathies & 710-719 & 0 & 0 & 3 & 6 & 9 \\
\hline -Dorsopathies & $720-724$ & 0 & 0 & 0 & 4 & 4 \\
\hline -Rheumatism, Excluding Back & 725-729 & 0 & 0 & 5 & 5 & 10 \\
\hline -Other Dis \& Acquired Deformities & 730-739 & 0 & 0 & 0 & 4 & 4 \\
\hline CONGENITAL ANOMALIES & $740-759$ & 0 & 0 & 0 & 1 & 1 \\
\hline $\begin{array}{l}\text { SYMPTOMS, SIGNS, \& ILL-DEFINED } \\
\text { CONDITIONS }\end{array}$ & $780-799$ & 0 & 1 & 5 & 6 & 12 \\
\hline -Symptoms & 780-789 & 0 & 0 & 4 & 6 & 10 \\
\hline -Non-Specific Abnormal Findings & $790-796$ & 0 & 1 & 1 & 0 & 2 \\
\hline -Ill-Defined \& Unknown Causes & 797-799 & 0 & 0 & 0 & 0 & 0 \\
\hline INJURY \& POISONING & $800-999$ & 0 & 0 & 2 & 10 & 12 \\
\hline -Fracture - Neck, Trunk & $805-809$ & 0 & 0 & 0 & 1 & 1 \\
\hline -Fracture - Upper Limb & 810-819 & 0 & 0 & 0 & 0 & 0 \\
\hline -Fracture - Lower Limb & $820-829$ & 0 & 0 & 1 & 2 & 3 \\
\hline -Dislocation & 830-839 & 0 & 0 & 0 & 1 & 1 \\
\hline -Sprains \& Strains - Other & $840-845,848$ & 0 & 0 & 1 & 3 & 4 \\
\hline -Internal Injury - Thorax, Abdomen, Pelvis & $860-869$ & 0 & 0 & 0 & 0 & 0 \\
\hline -Open Wound - Lower Limb & 890-897 & 0 & 0 & 0 & 0 & 0 \\
\hline -Late Effects of Accident & $905-909$ & 0 & 0 & 0 & 1 & 1 \\
\hline -Superficial Injury & $910-919$ & 0 & 0 & 0 & 0 & 0 \\
\hline -Contusion & $920-924$ & 0 & 0 & 0 & 0 & 0 \\
\hline -Burns & \begin{tabular}{|c|}
$940-949$ \\
\end{tabular} & 0 & 0 & 0 & 1 & 1 \\
\hline -Complications \& Unspecified Injuries & 958-959 & 0 & 0 & 0 & 0 & 0 \\
\hline
\end{tabular}

(Continued)

*Only those diagnostic categories and gender/age combinations with at least one occurrence appear in this table. 


\section{Kansas City Plant 2007}

Absence Data

Appendix G. Number of Diagnoses in Each Diagnostic Category by Gender and Age*

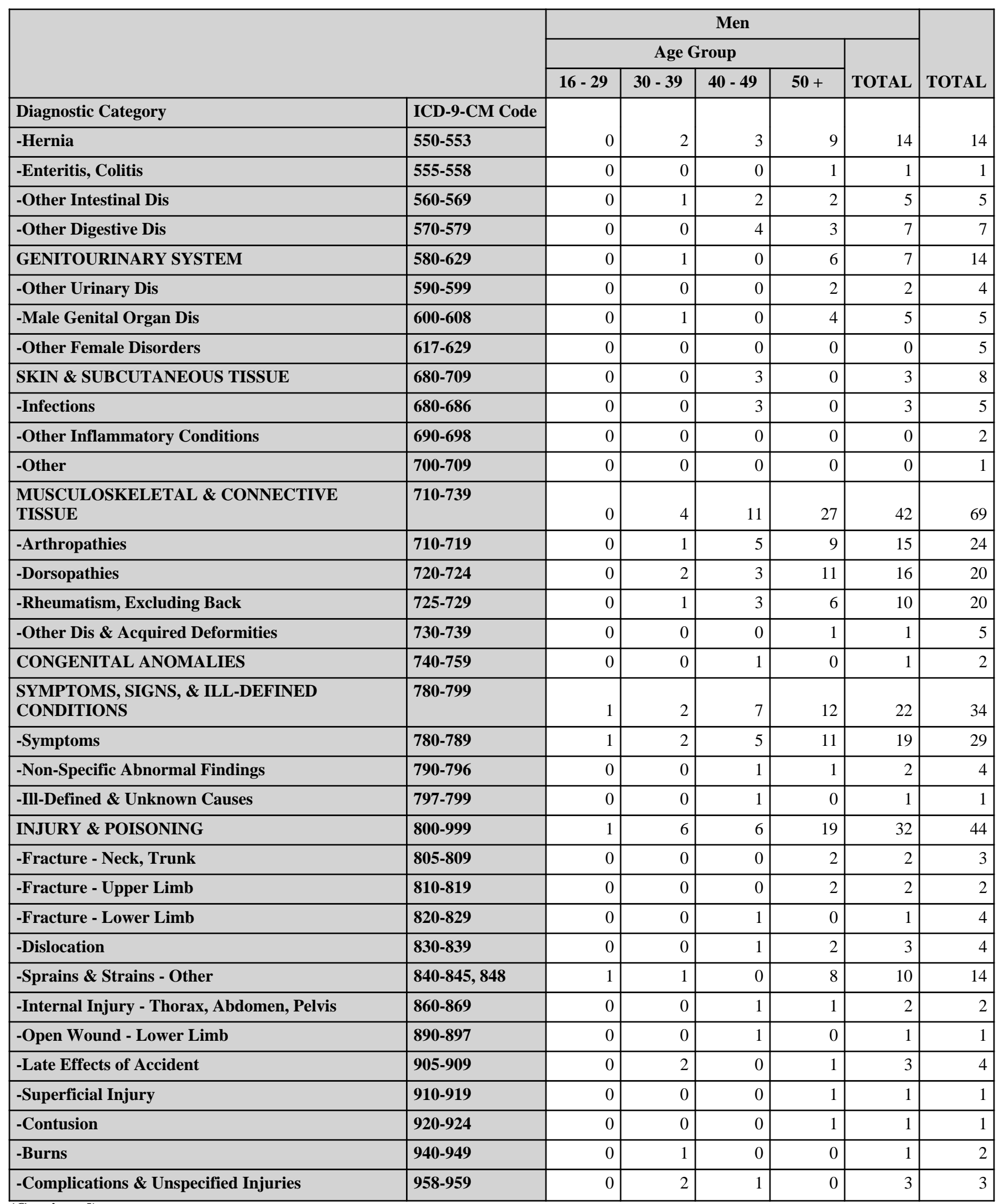

(Continued)

*Only those diagnostic categories and gender/age combinations with at least one occurrence appear in this table. 


\section{Kansas City Plant 2007}

Absence Data

Appendix G. Number of Diagnoses in Each Diagnostic Category by Gender and Age*

\begin{tabular}{|c|c|c|c|c|c|c|}
\hline & \multicolumn{5}{|c|}{ Women } \\
\hline & & \multicolumn{4}{|c|}{ Age Group } & \multirow[b]{2}{*}{ TOTAL } \\
\hline & & $16-29$ & $30-39$ & $40-49$ & $50+$ & \\
\hline Diagnostic Category & ICD-9-CM Code & \multirow[b]{2}{*}{0} & \multirow[b]{2}{*}{0} & \multirow[b]{2}{*}{0} & \multirow[b]{2}{*}{1} & \multirow[b]{2}{*}{1} \\
\hline -Complications of Surgical/Medical Care & 996-999 & & & & & \\
\hline $\begin{array}{l}\text { HEALTH STATUS/HEALTH SERVICE } \\
\text { CONTACT }\end{array}$ & V01-V82 & 0 & 0 & 1 & 0 & 1 \\
\hline -Personal \& Family History & V10-V19 & 0 & 0 & 0 & 0 & 0 \\
\hline -Specific Procedures/Aftercare & V50-V59 & 0 & 0 & 1 & 0 & 1 \\
\hline -Examination \& Investigation & V70-V82 & 0 & 0 & 0 & 0 & 0 \\
\hline
\end{tabular}

*Only those diagnostic categories and gender/age combinations with at least one occurrence appear in this table. 


\section{Kansas City Plant 2007}

Absence Data

Appendix G. Number of Diagnoses in Each Diagnostic Category by Gender and Age*

\begin{tabular}{|c|c|c|c|c|c|c|c|}
\hline & & \multicolumn{5}{|c|}{ Men } & \multirow[b]{3}{*}{ TOTAL } \\
\hline & & \multicolumn{4}{|c|}{ Age Group } & \multirow[b]{2}{*}{ TOTAL } & \\
\hline & & $16-29$ & $30-39$ & $40-49$ & $50+$ & & \\
\hline -Complications of Surgical/Medical Care & 996-999 & 0 & 0 & 1 & 1 & 2 & 3 \\
\hline $\begin{array}{l}\text { HEALTH STATUS/HEALTH SERVICE } \\
\text { CONTACT }\end{array}$ & V01-V82 & 0 & 0 & 2 & 3 & 5 & 6 \\
\hline -Personal \& Family History & V10-V19 & 0 & 0 & 1 & 3 & 4 & 4 \\
\hline -Specific Procedures/Aftercare & V50-V59 & 0 & 0 & 0 & 0 & 0 & 1 \\
\hline -Examination \& Investigation & V70-V82 & 0 & 0 & 1 & 0 & 1 & 1 \\
\hline
\end{tabular}

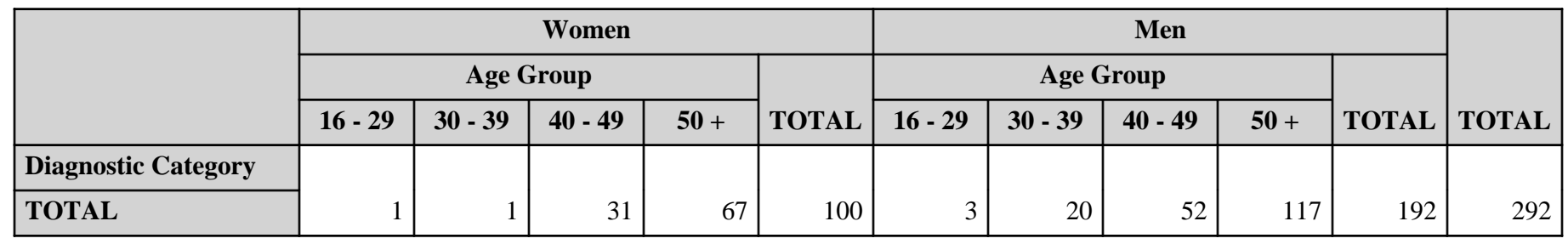

*Only those diagnostic categories and gender/age combinations with at least one occurrence appear in this table. 


\section{Kansas City Plant 2007}

Absence Data

Appendix H. Total Number of Calendar Days Absent in Each Diagnostic Category by Gender and Age*

\begin{tabular}{|c|c|c|c|c|c|c|}
\hline & & \multicolumn{5}{|c|}{ Women } \\
\hline & & \multicolumn{4}{|c|}{ Age Group } & \multirow[b]{2}{*}{ TOTAL } \\
\hline & & $16-29$ & $30-39$ & $40-49$ & $50+$ & \\
\hline Diagnostic Category & ICD-9-CM Code & \multirow[b]{2}{*}{0} & \multirow[b]{2}{*}{0} & \multirow[b]{2}{*}{0} & \multirow[b]{2}{*}{31} & \multirow[b]{2}{*}{31} \\
\hline INFECTIOUS \& PARASITIC DISEASES (DIS) & 001-139 & & & & & \\
\hline MALIGNANT NEOPLASMS & 140-208, 230-234 & 0 & 0 & 0 & 0 & 0 \\
\hline BENIGN \& UNCERTAIN NEOPLASMS & 210-229, 235-239 & 0 & 0 & 0 & 15 & 15 \\
\hline ENDOCRINE/METABOLIC/IMMUNITY & $240-279$ & 0 & 0 & 18 & 14 & 32 \\
\hline BLOOD \& BLOOD-FORMING ORGANS & $280-289$ & 0 & 0 & 47 & 25 & 72 \\
\hline MENTAL DISORDERS & $290-319$ & 0 & 0 & 17 & 0 & 17 \\
\hline NERVOUS SYSTEM (NS) \& SENSE ORGANS & $320-389$ & 0 & 0 & 8 & 351 & 359 \\
\hline CIRCULATORY SYSTEM & $390-459$ & 0 & 0 & 57 & 107 & 164 \\
\hline RESPIRATORY SYSTEM & $460-519$ & 18 & 0 & 12 & 109 & 139 \\
\hline DIGESTIVE SYSTEM & $520-579$ & 0 & 0 & 10 & 0 & 10 \\
\hline GENITOURINARY SYSTEM & $580-629$ & 0 & 0 & 20 & 102 & 122 \\
\hline SKIN \& SUBCUTANEOUS TISSUE & 680-709 & 0 & 0 & 13 & 116 & 129 \\
\hline $\begin{array}{l}\text { MUSCULOSKELETAL \& CONNECTIVE } \\
\text { TISSUE }\end{array}$ & $710-739$ & 0 & 0 & 91 & 992 & 1,083 \\
\hline CONGENITAL ANOMALIES & $740-759$ & 0 & 0 & 0 & 20 & 20 \\
\hline $\begin{array}{l}\text { SYMPTOMS, SIGNS, \& ILL-DEFINED } \\
\text { CONDITIONS }\end{array}$ & \begin{tabular}{|l|}
$780-799$ \\
\end{tabular} & 0 & 3 & 83 & 231 & 317 \\
\hline INJURY \& POISONING & $800-999$ & 0 & 0 & 30 & 532 & 562 \\
\hline
\end{tabular}

\footnotetext{
*Absences with >1 ICD-9-CM code in the same diagnostic category were counted only once. Only those diagnostic categories and gender/age combinations with at least one occurrence appear in this table.
} 


\section{Kansas City Plant 2007}

Absence Data

Appendix H. Total Number of Calendar Days Absent in Each Diagnostic Category by Gender and Age*

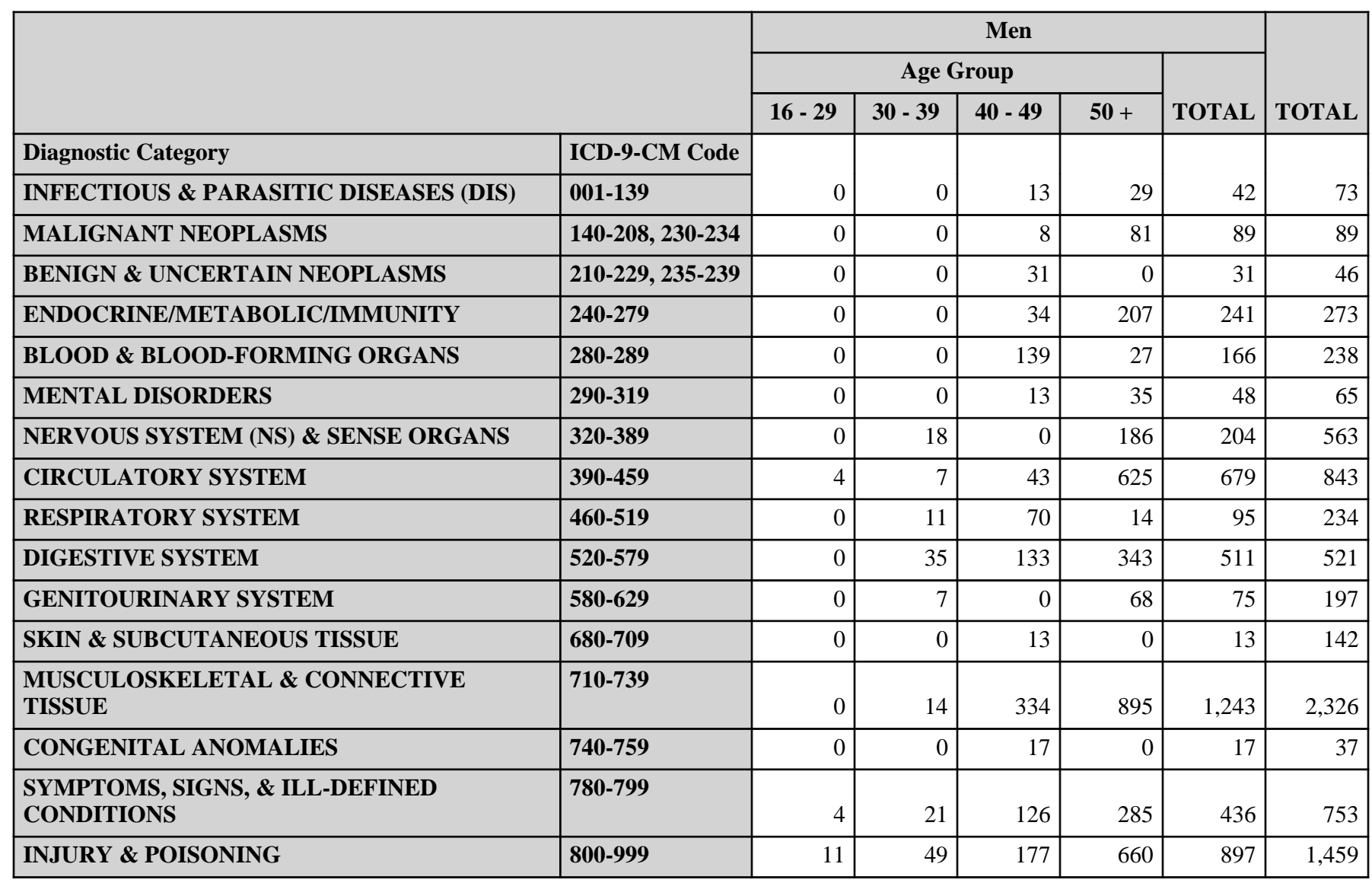

\footnotetext{
*Absences with >1 ICD-9-CM code in the same diagnostic category were counted only once. Only those diagnostic categories and gender/age combinations with at least one occurrence appear in this table.
} 


\section{Kansas City Plant 2007}

Absence Data

Appendix I. Number of Diagnoses in Each Diagnostic Category by Gender and Job Category*

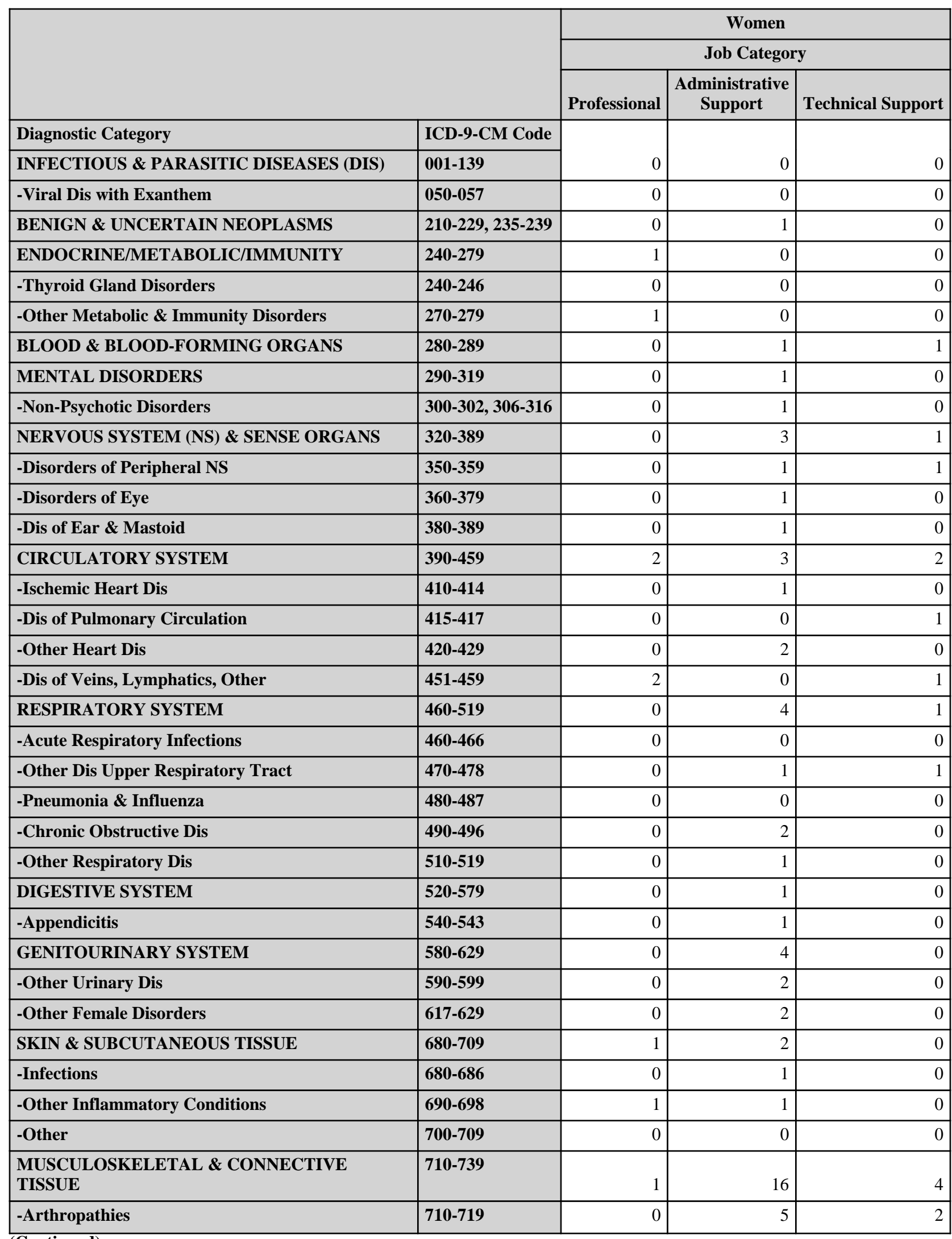

(Continued)

*Only those diagnostic categories and gender/job category combinations with at least one occurrence appear in this table. 


\section{Kansas City Plant 2007}

Absence Data

Appendix I. Number of Diagnoses in Each Diagnostic Category by Gender and Job Category*

\begin{tabular}{|c|c|c|c|c|c|}
\hline & & \multicolumn{4}{|c|}{ Women } \\
\hline & & \multicolumn{3}{|c|}{ Job Category } & \multirow[b]{2}{*}{ TOTAL } \\
\hline & & Service & Security and Fire & Line Operators & \\
\hline Diagnostic Category & ICD-9-CM Code & \multirow[b]{2}{*}{0} & \multirow[b]{2}{*}{0} & \multirow[b]{2}{*}{1} & \multirow[b]{2}{*}{1} \\
\hline INFECTIOUS \& PARASITIC DISEASES (DIS) & 001-139 & & & & \\
\hline -Viral Dis with Exanthem & 050-057 & 0 & 0 & 1 & 1 \\
\hline BENIGN \& UNCERTAIN NEOPLASMS & 210-229, 235-239 & 0 & 0 & 0 & 1 \\
\hline ENDOCRINE/METABOLIC/IMMUNITY & $240-279$ & 0 & 1 & 0 & 2 \\
\hline -Thyroid Gland Disorders & $240-246$ & 0 & 1 & 0 & 1 \\
\hline -Other Metabolic \& Immunity Disorders & 270-279 & 0 & 0 & 0 & 1 \\
\hline BLOOD \& BLOOD-FORMING ORGANS & $280-289$ & 0 & 0 & 0 & 2 \\
\hline MENTAL DISORDERS & 290-319 & 0 & 0 & 0 & 1 \\
\hline -Non-Psychotic Disorders & $300-302,306-316$ & 0 & 0 & 0 & 1 \\
\hline NERVOUS SYSTEM (NS) \& SENSE ORGANS & 320-389 & 0 & 0 & 2 & 6 \\
\hline -Disorders of Peripheral NS & $350-359$ & 0 & 0 & 2 & 4 \\
\hline -Disorders of Eye & $360-379$ & 0 & 0 & 0 & 1 \\
\hline -Dis of Ear \& Mastoid & 380-389 & 0 & 0 & 0 & 1 \\
\hline CIRCULATORY SYSTEM & $390-459$ & 0 & 0 & 0 & 7 \\
\hline -Ischemic Heart Dis & $410-414$ & 0 & 0 & 0 & 1 \\
\hline -Dis of Pulmonary Circulation & $415-417$ & 0 & 0 & 0 & 1 \\
\hline -Other Heart Dis & $420-429$ & 0 & 0 & 0 & 2 \\
\hline -Dis of Veins, Lymphatics, Other & 451-459 & 0 & 0 & 0 & 3 \\
\hline RESPIRATORY SYSTEM & $460-519$ & 2 & 2 & 5 & 14 \\
\hline -Acute Respiratory Infections & $460-466$ & 1 & 1 & 0 & 2 \\
\hline -Other Dis Upper Respiratory Tract & $470-478$ & 0 & 0 & 3 & 5 \\
\hline -Pneumonia \& Influenza & $480-487$ & 1 & 0 & 1 & 2 \\
\hline -Chronic Obstructive Dis & $490-496$ & 0 & 1 & 1 & $\overline{4}$ \\
\hline -Other Respiratory Dis & $510-519$ & 0 & 0 & 0 & 1 \\
\hline DIGESTIVE SYSTEM & $520-579$ & 0 & 0 & 0 & 1 \\
\hline -Appendicitis & $540-543$ & 0 & 0 & 0 & 1 \\
\hline GENITOURINARY SYSTEM & $580-629$ & 0 & 0 & 3 & 7 \\
\hline -Other Urinary Dis & $590-599$ & 0 & 0 & 0 & 2 \\
\hline -Other Female Disorders & $617-629$ & 0 & 0 & 3 & 5 \\
\hline SKIN \& SUBCUTANEOUS TISSUE & $680-709$ & 0 & 0 & 2 & 5 \\
\hline -Infections & $680-686$ & 0 & 0 & 1 & 2 \\
\hline -Other Inflammatory Conditions & $690-698$ & 0 & 0 & 0 & 2 \\
\hline -Other & 700-709 & 0 & 0 & 1 & 1 \\
\hline $\begin{array}{l}\text { MUSCULOSKELETAL \& CONNECTIVE } \\
\text { TISSUE }\end{array}$ & $710-739$ & 2 & 0 & 4 & 27 \\
\hline -Arthropathies & $710-719$ & 0 & 0 & 2 & 9 \\
\hline
\end{tabular}

(Continued)

*Only those diagnostic categories and gender/job category combinations with at least one occurrence appear in this table. 


\section{Kansas City Plant 2007}

Absence Data

Appendix I. Number of Diagnoses in Each Diagnostic Category by Gender and Job Category*

\begin{tabular}{|c|c|c|c|c|}
\hline & & \multicolumn{3}{|c|}{ Women } \\
\hline & & \multicolumn{3}{|c|}{ Job Category } \\
\hline & & Professional & $\begin{array}{c}\text { Administrative } \\
\text { Support }\end{array}$ & Technical Support \\
\hline Diagnostic Category & ICD-9-CM Code & \multirow[b]{2}{*}{0} & \multirow[b]{2}{*}{3} & \multirow[b]{2}{*}{0} \\
\hline -Dorsopathies & $720-724$ & & & \\
\hline -Rheumatism, Excluding Back & $725-729$ & 1 & 5 & 2 \\
\hline -Other Dis \& Acquired Deformities & $730-739$ & 0 & 3 & 0 \\
\hline CONGENITAL ANOMALIES & $740-759$ & 0 & 1 & $\overline{0}$ \\
\hline $\begin{array}{l}\text { SYMPTOMS, SIGNS, \& ILL-DEFINED } \\
\text { CONDITIONS }\end{array}$ & $780-799$ & 0 & 6 & 3 \\
\hline -Symptoms & $780-789$ & 0 & 5 & 2 \\
\hline -Non-Specific Abnormal Findings & $790-796$ & 0 & 1 & 1 \\
\hline INJURY \& POISONING & $800-999$ & 0 & 1 & 1 \\
\hline -Fracture - Neck, Trunk & 805-809 & 0 & 0 & 0 \\
\hline -Fracture - Lower Limb & $820-829$ & 0 & 0 & 0 \\
\hline -Dislocation & $830-839$ & 0 & 0 & 0 \\
\hline -Sprains \& Strains - Other & $840-845,848$ & 0 & 1 & 1 \\
\hline -Late Effects of Accident & 905-909 & 0 & 0 & 0 \\
\hline -Burns & $940-949$ & 0 & 0 & 0 \\
\hline -Complications of Surgical/Medical Care & 996-999 & 0 & 0 & 0 \\
\hline $\begin{array}{l}\text { HEALTH STATUS/HEALTH SERVICE } \\
\text { CONTACT }\end{array}$ & V01-V82 & 0 & 1 & 0 \\
\hline -Specific Procedures/Aftercare & V50-V59 & 0 & 1 & 0 \\
\hline
\end{tabular}

*Only those diagnostic categories and gender/job category combinations with at least one occurrence appear in this table. 


\section{Kansas City Plant 2007}

Absence Data

Appendix I. Number of Diagnoses in Each Diagnostic Category by Gender and Job Category*

\begin{tabular}{|c|c|c|c|c|c|}
\hline & & \multicolumn{4}{|c|}{ Women } \\
\hline & & \multicolumn{3}{|c|}{ Job Category } & \multirow[b]{2}{*}{ TOTAL } \\
\hline & & Service & Security and Fire & Line Operators & \\
\hline Diagnostic Category & ICD-9-CM Code & \multirow[b]{2}{*}{1} & \multirow[b]{2}{*}{0} & \multirow[b]{2}{*}{0} & \multirow[b]{2}{*}{4} \\
\hline -Dorsopathies & $720-724$ & & & & \\
\hline -Rheumatism, Excluding Back & $725-729$ & 1 & 0 & 1 & 10 \\
\hline -Other Dis \& Acquired Deformities & $730-739$ & 0 & 0 & 1 & 4 \\
\hline CONGENITAL ANOMALIES & $740-759$ & 0 & 0 & 0 & 1 \\
\hline $\begin{array}{l}\text { SYMPTOMS, SIGNS, \& ILL-DEFINED } \\
\text { CONDITIONS }\end{array}$ & $780-799$ & 0 & 0 & 3 & 12 \\
\hline -Symptoms & 780-789 & 0 & 0 & 3 & 10 \\
\hline -Non-Specific Abnormal Findings & $790-796$ & 0 & 0 & 0 & 2 \\
\hline INJURY \& POISONING & $800-999$ & 3 & 3 & 4 & 12 \\
\hline -Fracture - Neck, Trunk & $805-809$ & 1 & 0 & 0 & 1 \\
\hline -Fracture - Lower Limb & $820-829$ & 0 & 1 & 2 & 3 \\
\hline -Dislocation & $830-839$ & 1 & 0 & 0 & 1 \\
\hline -Sprains \& Strains - Other & $840-845,848$ & 1 & 1 & 0 & 4 \\
\hline -Late Effects of Accident & $905-909$ & 0 & 0 & 1 & 1 \\
\hline -Burns & 940-949 & 0 & 0 & 1 & 1 \\
\hline -Complications of Surgical/Medical Care & 996-999 & 0 & 1 & 0 & 1 \\
\hline $\begin{array}{l}\text { HEALTH STATUS/HEALTH SERVICE } \\
\text { CONTACT }\end{array}$ & V01-V82 & 0 & 0 & 0 & 1 \\
\hline -Specific Procedures/Aftercare & V50-V59 & 0 & 0 & 0 & 1 \\
\hline
\end{tabular}

\begin{tabular}{|c|c|c|c|c|c|c|c|}
\hline & \multicolumn{7}{|c|}{ Women } \\
\hline & \multicolumn{6}{|c|}{ Job Category } & \multirow[b]{2}{*}{ TOTAL } \\
\hline & Professional & $\begin{array}{c}\text { Administrative } \\
\text { Support }\end{array}$ & Technical Support & Service & Security and Fire & Line Operators & \\
\hline Diagnostic Category & \multirow[b]{2}{*}{5} & \multirow[b]{2}{*}{45} & \multirow[b]{2}{*}{13} & \multirow[b]{2}{*}{7} & \multirow[b]{2}{*}{6} & \multirow[b]{2}{*}{24} & \multirow[b]{2}{*}{100} \\
\hline TOTAL & & & & & & & \\
\hline
\end{tabular}

*Only those diagnostic categories and gender/job category combinations with at least one occurrence appear in this table. 


\section{Kansas City Plant 2007}

Absence Data

Appendix I. Number of Diagnoses in Each Diagnostic Category by Gender and Job Category*

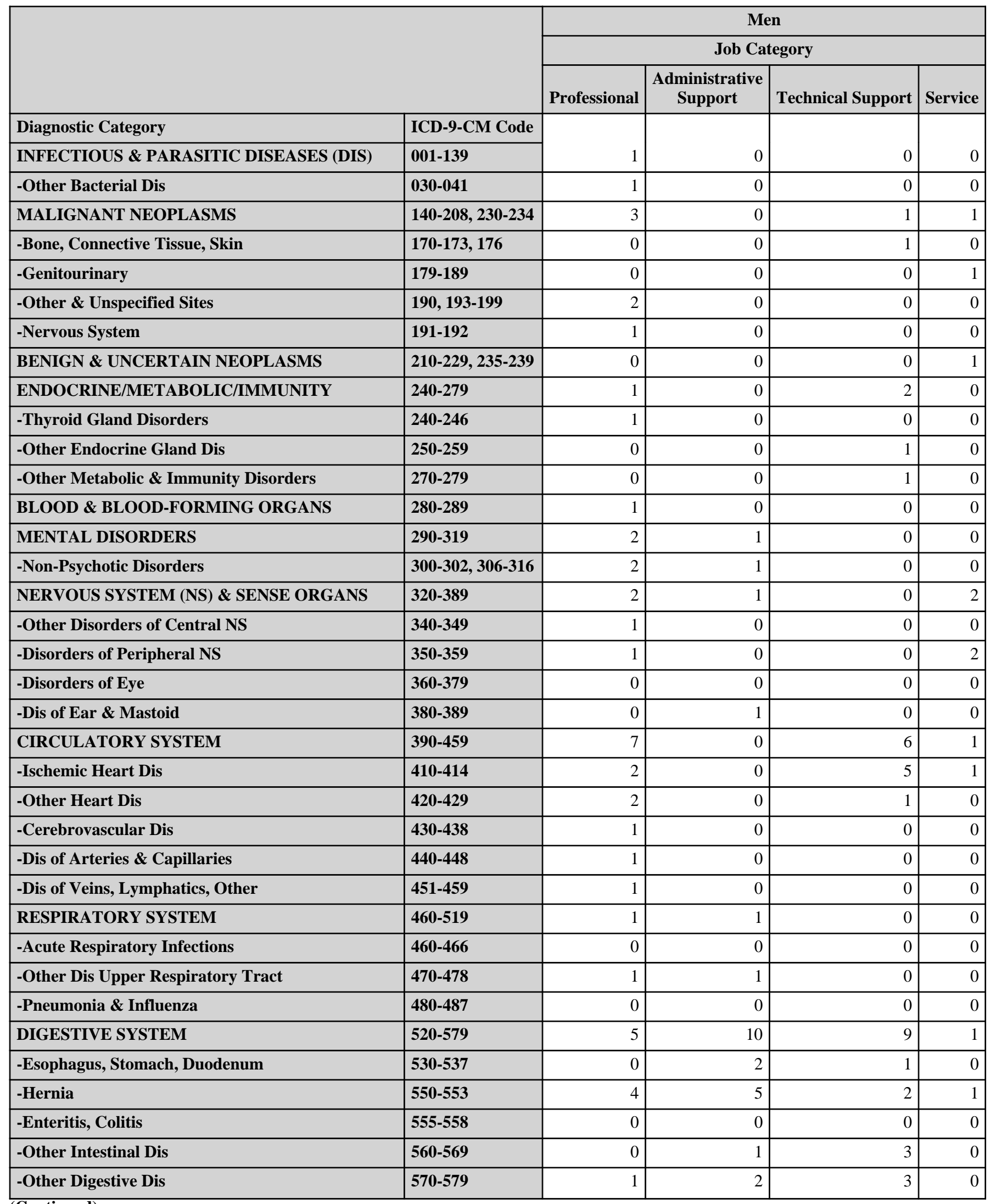

(Continued)

*Only those diagnostic categories and gender/job category combinations with at least one occurrence appear in this table. 


\section{Kansas City Plant 2007}

Absence Data

Appendix I. Number of Diagnoses in Each Diagnostic Category by Gender and Job Category*

\begin{tabular}{|c|c|c|c|c|c|}
\hline & & \multicolumn{4}{|c|}{ Men } \\
\hline & & \multicolumn{3}{|c|}{ Job Category } & \multirow[b]{2}{*}{ TOTAL } \\
\hline & & Security and Fire & Crafts & Line Operators & \\
\hline Diagnostic Category & ICD-9-CM Code & \multirow[b]{2}{*}{1} & \multirow[b]{2}{*}{0} & \multirow[b]{2}{*}{0} & \multirow[b]{2}{*}{2} \\
\hline INFECTIOUS \& PARASITIC DISEASES (DIS) & 001-139 & & & & \\
\hline -Other Bacterial Dis & $030-041$ & 1 & 0 & 0 & 2 \\
\hline MALIGNANT NEOPLASMS & 140-208, 230-234 & 0 & 0 & 0 & 5 \\
\hline -Bone, Connective Tissue, Skin & $170-173,176$ & 0 & 0 & 0 & 1 \\
\hline -Genitourinary & 179-189 & 0 & 0 & 0 & 1 \\
\hline -Other \& Unspecified Sites & 190, 193-199 & 0 & 0 & 0 & 2 \\
\hline -Nervous System & 191-192 & 0 & 0 & 0 & 1 \\
\hline BENIGN \& UNCERTAIN NEOPLASMS & 210-229, 235-239 & 0 & 0 & 0 & 1 \\
\hline ENDOCRINE/METABOLIC/IMMUNITY & 240-279 & 0 & 0 & 0 & 3 \\
\hline -Thyroid Gland Disorders & $240-246$ & 0 & 0 & 0 & 1 \\
\hline -Other Endocrine Gland Dis & $250-259$ & 0 & 0 & 0 & 1 \\
\hline -Other Metabolic \& Immunity Disorders & $270-279$ & 0 & 0 & 0 & 1 \\
\hline BLOOD \& BLOOD-FORMING ORGANS & 280-289 & 0 & 2 & 0 & 3 \\
\hline MENTAL DISORDERS & $290-319$ & 0 & 0 & 0 & 3 \\
\hline -Non-Psychotic Disorders & $300-302,306-316$ & 0 & 0 & 0 & 3 \\
\hline NERVOUS SYSTEM (NS) \& SENSE ORGANS & $320-389$ & 0 & 2 & 1 & 8 \\
\hline -Other Disorders of Central NS & 340-349 & 0 & 0 & 0 & 1 \\
\hline -Disorders of Peripheral NS & $350-359$ & 0 & 2 & 0 & 5 \\
\hline -Disorders of Eye & $360-379$ & 0 & 0 & 1 & 1 \\
\hline -Dis of Ear \& Mastoid & $380-389$ & 0 & 0 & 0 & 1 \\
\hline CIRCULATORY SYSTEM & $390-459$ & 1 & 1 & 1 & 17 \\
\hline -Ischemic Heart Dis & $410-414$ & 1 & 0 & 0 & 9 \\
\hline -Other Heart Dis & $420-429$ & 0 & 1 & 0 & 4 \\
\hline -Cerebrovascular Dis & $430-438$ & 0 & 0 & 0 & 1 \\
\hline -Dis of Arteries \& Capillaries & $440-448$ & 0 & 0 & 0 & 1 \\
\hline -Dis of Veins, Lymphatics, Other & 451-459 & 0 & 0 & 1 & 2 \\
\hline RESPIRATORY SYSTEM & $460-519$ & 0 & 2 & 2 & 6 \\
\hline -Acute Respiratory Infections & $460-466$ & 0 & 0 & 1 & 1 \\
\hline -Other Dis Upper Respiratory Tract & $470-478$ & 0 & 0 & 0 & 2 \\
\hline -Pneumonia \& Influenza & $480-487$ & 0 & 2 & 1 & 3 \\
\hline DIGESTIVE SYSTEM & $520-579$ & 0 & 4 & 3 & 32 \\
\hline -Esophagus, Stomach, Duodenum & $530-537$ & 0 & 2 & 0 & 5 \\
\hline -Hernia & $550-553$ & 0 & 1 & 1 & 14 \\
\hline -Enteritis, Colitis & \begin{tabular}{|l|}
$555-558$ \\
\end{tabular} & 0 & 0 & 1 & 1 \\
\hline -Other Intestinal Dis & $560-569$ & 0 & 0 & 1 & 5 \\
\hline -Other Digestive Dis & $570-579$ & 0 & 1 & 0 & 7 \\
\hline
\end{tabular}

(Continued)

*Only those diagnostic categories and gender/job category combinations with at least one occurrence appear in this table. 


\section{Kansas City Plant 2007}

Absence Data

Appendix I. Number of Diagnoses in Each Diagnostic Category by Gender and Job Category*

\begin{tabular}{|c|c|c|c|c|c|}
\hline & \multicolumn{4}{|c|}{ Men } \\
\hline & & \multicolumn{4}{|c|}{ Job Category } \\
\hline & & Professional & $\begin{array}{c}\text { Administrative } \\
\text { Support }\end{array}$ & Technical Support & Service \\
\hline Diagnostic Category & ICD-9-CM Code & \multirow[b]{2}{*}{1} & \multirow[b]{2}{*}{0} & \multirow[b]{2}{*}{2} & \multirow[b]{2}{*}{1} \\
\hline GENITOURINARY SYSTEM & $580-629$ & & & & \\
\hline -Male Genital Organ Dis & $600-608$ & 1 & 0 & 2 & 1 \\
\hline SKIN \& SUBCUTANEOUS TISSUE & $680-709$ & 3 & 0 & 0 & 0 \\
\hline -Infections & $680-686$ & 3 & 0 & 0 & 0 \\
\hline $\begin{array}{l}\text { MUSCULOSKELETAL \& CONNECTIVE } \\
\text { TISSUE }\end{array}$ & 710-739 & 17 & 5 & 6 & 3 \\
\hline -Rheumatism, Excluding Back & 725-729 & 3 & 2 & 1 & 1 \\
\hline -Other Dis \& Acquired Deformities & $730-739$ & 0 & 0 & 1 & 0 \\
\hline CONGENITAL ANOMALIES & $740-759$ & 1 & 0 & 0 & 0 \\
\hline $\begin{array}{l}\text { SYMPTOMS, SIGNS, \& ILL-DEFINED } \\
\text { CONDITIONS }\end{array}$ & $780-799$ & 9 & 1 & 5 & 2 \\
\hline -Symptoms & 780-789 & 7 & 1 & 5 & 1 \\
\hline -Non-Specific Abnormal Findings & $790-796$ & 1 & 0 & 0 & 1 \\
\hline -Ill-Defined \& Unknown Causes & 797-799 & 1 & 0 & 0 & 0 \\
\hline INJURY \& POISONING & $800-999$ & 11 & 2 & 7 & 1 \\
\hline -Internal Injury - Thorax, Abdomen, Pelvis & $860-869$ & 1 & 0 & 1 & 0 \\
\hline -Open Wound - Lower Limb & $890-897$ & 0 & 0 & 1 & 0 \\
\hline -Late Effects of Accident & $905-909$ & 1 & 0 & 0 & 0 \\
\hline -Superficial Injury & $910-919$ & 0 & 0 & 0 & 0 \\
\hline -Contusion & $920-924$ & 1 & 0 & 0 & 0 \\
\hline -Burns & 940-949 & 1 & 0 & 0 & 0 \\
\hline -Complications \& Unspecified Injuries & 958-959 & 2 & 0 & 0 & 0 \\
\hline -Complications of Surgical/Medical Care & 996-999 & 0 & 0 & 0 & 0 \\
\hline $\begin{array}{l}\text { HEALTH STATUS/HEALTH SERVICE } \\
\text { CONTACT }\end{array}$ & V01-V82 & 0 & 0 & 2 & 0 \\
\hline -Personal \& Family History & V10-V19 & 0 & 0 & 2 & 0 \\
\hline -Examination \& Investigation & V70-V82 & 0 & 0 & 0 & 0 \\
\hline
\end{tabular}

*Only those diagnostic categories and gender/job category combinations with at least one occurrence appear in this table. 


\section{Kansas City Plant 2007}

Absence Data

Appendix I. Number of Diagnoses in Each Diagnostic Category by Gender and Job Category*

\begin{tabular}{|c|c|c|c|c|c|}
\hline & & \multicolumn{4}{|c|}{ Men } \\
\hline & & \multicolumn{3}{|c|}{ Job Category } & \multirow[b]{2}{*}{ TOTAL } \\
\hline & & Security and Fire & Crafts & Line Operators & \\
\hline Diagnostic Category & ICD-9-CM Code & \multirow[b]{2}{*}{2} & \multirow[b]{2}{*}{0} & \multirow[b]{2}{*}{1} & \multirow[b]{2}{*}{7} \\
\hline GENITOURINARY SYSTEM & $580-629$ & & & & \\
\hline -Other Urinary Dis & $590-599$ & 1 & 0 & 1 & 2 \\
\hline -Male Genital Organ Dis & $600-608$ & 1 & 0 & 0 & 5 \\
\hline SKIN \& SUBCUTANEOUS TISSUE & 680-709 & 0 & 0 & 0 & 3 \\
\hline -Infections & $680-686$ & 0 & 0 & 0 & 3 \\
\hline $\begin{array}{l}\text { MUSCULOSKELETAL \& CONNECTIVE } \\
\text { TISSUE }\end{array}$ & $710-739$ & 6 & 3 & 2 & 42 \\
\hline -Arthropathies & $710-719$ & 1 & 1 & 1 & 15 \\
\hline -Dorsopathies & $720-724$ & 4 & 0 & 1 & 16 \\
\hline -Rheumatism, Excluding Back & $725-729$ & 1 & 2 & 0 & 10 \\
\hline -Other Dis \& Acquired Deformities & $730-739$ & 0 & 0 & 0 & 1 \\
\hline CONGENITAL ANOMALIES & $740-759$ & 0 & 0 & 0 & 1 \\
\hline $\begin{array}{l}\text { SYMPTOMS, SIGNS, \& ILL-DEFINED } \\
\text { CONDITIONS }\end{array}$ & 780-799 & 2 & 2 & 1 & 22 \\
\hline -Symptoms & 780-789 & 2 & 2 & 1 & 19 \\
\hline -Non-Specific Abnormal Findings & $790-796$ & 0 & 0 & 0 & 2 \\
\hline -Ill-Defined \& Unknown Causes & 797-799 & 0 & 0 & 0 & 1 \\
\hline INJURY \& POISONING & $800-999$ & 1 & 5 & 5 & 32 \\
\hline -Fracture - Neck, Trunk & 805-809 & 0 & 0 & 0 & 2 \\
\hline -Fracture - Upper Limb & $810-819$ & 0 & 0 & 0 & 2 \\
\hline -Fracture - Lower Limb & $820-829$ & 0 & 0 & 1 & 1 \\
\hline -Dislocation & $830-839$ & 0 & 1 & 0 & 3 \\
\hline -Sprains \& Strains - Other & $840-845,848$ & 1 & 1 & 1 & 10 \\
\hline -Internal Injury - Thorax, Abdomen, Pelvis & $860-869$ & 0 & 0 & 0 & 2 \\
\hline -Open Wound - Lower Limb & $890-897$ & 0 & 0 & 0 & 1 \\
\hline -Late Effects of Accident & 905-909 & 0 & 0 & 2 & 3 \\
\hline -Superficial Injury & 910-919 & 0 & 1 & 0 & 1 \\
\hline -Contusion & $920-924$ & 0 & 0 & 0 & 1 \\
\hline -Burns & 940-949 & 0 & 0 & 0 & 1 \\
\hline -Complications \& Unspecified Injuries & $958-959$ & 0 & 0 & 1 & 3 \\
\hline -Complications of Surgical/Medical Care & 996-999 & 0 & 2 & 0 & 2 \\
\hline $\begin{array}{l}\text { HEALTH STATUS/HEALTH SERVICE } \\
\text { CONTACT }\end{array}$ & V01-V82 & 0 & 3 & 0 & 5 \\
\hline -Personal \& Family History & V10-V19 & 0 & 2 & 0 & $\overline{4}$ \\
\hline -Examination \& Investigation & V70-V82 & 0 & 1 & 0 & 1 \\
\hline
\end{tabular}

*Only those diagnostic categories and gender/job category combinations with at least one occurrence appear in this table. 
Kansas City Plant 2007

Absence Data

Appendix I. Number of Diagnoses in Each Diagnostic Category by Gender and Job Category*

\begin{tabular}{|c|c|c|c|c|c|c|c|c|}
\hline & \multicolumn{8}{|c|}{ Men } \\
\hline & \multicolumn{7}{|c|}{ Job Category } & \multirow[b]{2}{*}{ TOTAL } \\
\hline & Professional & $\begin{array}{c}\text { Administrative } \\
\text { Support }\end{array}$ & $\begin{array}{c}\text { Technical } \\
\text { Support }\end{array}$ & Service & $\begin{array}{l}\text { Security and } \\
\text { Fire }\end{array}$ & Crafts & $\begin{array}{c}\text { Line } \\
\text { Operators }\end{array}$ & \\
\hline Diagnostic Category & \multirow[b]{2}{*}{65} & \multirow[b]{2}{*}{21} & \multirow[b]{2}{*}{40} & \multirow[b]{2}{*}{13} & \multirow[b]{2}{*}{13} & \multirow[b]{2}{*}{24} & \multirow[b]{2}{*}{16} & \multirow[b]{2}{*}{192} \\
\hline TOTAL & & & & & & & & \\
\hline
\end{tabular}

*Only those diagnostic categories and gender/job category combinations with at least one occurrence appear in this table. 


\section{Kansas City Plant 2007}

Absence Data

Appendix J. Total Number of Calendar Days Absent in Each Diagnostic Category by Gender and Job Category*

\begin{tabular}{|c|c|c|c|c|}
\hline & & \multicolumn{3}{|c|}{ Women } \\
\hline & & \multicolumn{3}{|c|}{ Job Category } \\
\hline & & Professional & $\begin{array}{l}\text { Administrative } \\
\text { Support }\end{array}$ & Technical Support \\
\hline Diagnostic Category & ICD-9-CM Code & \multirow[b]{2}{*}{0} & \multirow[b]{2}{*}{0} & \multirow[b]{2}{*}{0} \\
\hline INFECTIOUS \& PARASITIC DISEASES (DIS) & 001-139 & & & \\
\hline BENIGN \& UNCERTAIN NEOPLASMS & 210-229, 235-239 & 0 & 15 & $\overline{0}$ \\
\hline ENDOCRINE/METABOLIC/IMMUNITY & $240-279$ & 18 & 0 & 0 \\
\hline BLOOD \& BLOOD-FORMING ORGANS & $280-289$ & 0 & 25 & 47 \\
\hline MENTAL DISORDERS & $290-319$ & 0 & 17 & 0 \\
\hline NERVOUS SYSTEM (NS) \& SENSE ORGANS & $320-389$ & 0 & 43 & 129 \\
\hline CIRCULATORY SYSTEM & $390-459$ & 86 & 31 & 47 \\
\hline RESPIRATORY SYSTEM & $460-519$ & 0 & 36 & 15 \\
\hline DIGESTIVE SYSTEM & $520-579$ & 0 & 10 & 0 \\
\hline GENITOURINARY SYSTEM & $580-629$ & 0 & 60 & 0 \\
\hline SKIN \& SUBCUTANEOUS TISSUE & $680-709$ & 6 & 52 & 0 \\
\hline $\begin{array}{l}\text { MUSCULOSKELETAL \& CONNECTIVE } \\
\text { TISSUE }\end{array}$ & $710-739$ & 80 & 400 & 188 \\
\hline CONGENITAL ANOMALIES & $740-759$ & 0 & 20 & 0 \\
\hline $\begin{array}{l}\text { SYMPTOMS, SIGNS, \& ILL-DEFINED } \\
\text { CONDITIONS }\end{array}$ & $780-799$ & 0 & 89 & 62 \\
\hline INJURY \& POISONING & 800-999 & 0 & 12 & 129 \\
\hline
\end{tabular}

\footnotetext{
*Absences with >1 ICD-9-CM code in the same diagnostic category were counted only once. Only those diagnostic categories and gender/job category combinations with at least one occurrence appear in this table.
} 


\section{Kansas City Plant 2007}

Absence Data

Appendix J. Total Number of Calendar Days Absent in Each Diagnostic Category by Gender and Job Category*

\begin{tabular}{|c|c|c|c|c|c|}
\hline & & \multicolumn{4}{|c|}{ Women } \\
\hline & & \multicolumn{3}{|c|}{ Job Category } & \multirow[b]{2}{*}{ TOTAL } \\
\hline & & Service & Security and Fire & Line Operators & \\
\hline Diagnostic Category & ICD-9-CM Code & \multirow[b]{2}{*}{0} & \multirow[b]{2}{*}{0} & \multirow[b]{2}{*}{31} & \multirow[b]{2}{*}{31} \\
\hline INFECTIOUS \& PARASITIC DISEASES (DIS) & 001-139 & & & & \\
\hline BENIGN \& UNCERTAIN NEOPLASMS & 210-229, 235-239 & 0 & 0 & 0 & 15 \\
\hline ENDOCRINE/METABOLIC/IMMUNITY & $240-279$ & 0 & 14 & 0 & 32 \\
\hline BLOOD \& BLOOD-FORMING ORGANS & $280-289$ & 0 & 0 & 0 & 72 \\
\hline MENTAL DISORDERS & $290-319$ & 0 & 0 & 0 & 17 \\
\hline NERVOUS SYSTEM (NS) \& SENSE ORGANS & $320-389$ & 0 & 0 & 187 & 359 \\
\hline CIRCULATORY SYSTEM & $390-459$ & 0 & 0 & 0 & 164 \\
\hline RESPIRATORY SYSTEM & $460-519$ & 34 & 9 & 45 & 139 \\
\hline DIGESTIVE SYSTEM & $520-579$ & 0 & 0 & 0 & 10 \\
\hline GENITOURINARY SYSTEM & $580-629$ & 0 & 0 & 62 & 122 \\
\hline SKIN \& SUBCUTANEOUS TISSUE & $680-709$ & 0 & 0 & 71 & 129 \\
\hline $\begin{array}{l}\text { MUSCULOSKELETAL \& CONNECTIVE } \\
\text { TISSUE }\end{array}$ & $710-739$ & 83 & 0 & 332 & 1,083 \\
\hline CONGENITAL ANOMALIES & 740-759 & 0 & 0 & 0 & 20 \\
\hline $\begin{array}{l}\text { SYMPTOMS, SIGNS, \& ILL-DEFINED } \\
\text { CONDITIONS }\end{array}$ & $780-799$ & 0 & 0 & 166 & 317 \\
\hline INJURY \& POISONING & 800-999 & 105 & 131 & 185 & 562 \\
\hline
\end{tabular}

\footnotetext{
*Absences with >1 ICD-9-CM code in the same diagnostic category were counted only once. Only those diagnostic categories and gender/job category combinations with at least one occurrence appear in this table.
} 


\section{Kansas City Plant 2007}

Absence Data

Appendix J. Total Number of Calendar Days Absent in Each Diagnostic Category by Gender and Job Category*

\begin{tabular}{|c|c|c|c|c|c|}
\hline & & \multicolumn{4}{|c|}{ Men } \\
\hline & & \multicolumn{4}{|c|}{ Job Category } \\
\hline & & Professional & $\begin{array}{l}\text { Administrative } \\
\text { Support }\end{array}$ & Technical Support & Service \\
\hline Diagnostic Category & ICD-9-CM Code & \multirow[b]{2}{*}{13} & \multirow[b]{2}{*}{0} & \multirow[b]{2}{*}{0} & \multirow[b]{2}{*}{0} \\
\hline INFECTIOUS \& PARASITIC DISEASES (DIS) & 001-139 & & & & \\
\hline BENIGN \& UNCERTAIN NEOPLASMS & 210-229, 235-239 & 0 & 0 & 0 & 31 \\
\hline ENDOCRINE/METABOLIC/IMMUNITY & $240-279$ & 21 & 0 & 220 & 0 \\
\hline BLOOD \& BLOOD-FORMING ORGANS & 280-289 & 18 & 0 & 0 & 0 \\
\hline MENTAL DISORDERS & 290-319 & 24 & 24 & 0 & 0 \\
\hline DIGESTIVE SYSTEM & $520-579$ & 54 & 185 & 161 & 42 \\
\hline GENITOURINARY SYSTEM & $580-629$ & 7 & 0 & 12 & 21 \\
\hline SKIN \& SUBCUTANEOUS TISSUE & 680-709 & 13 & 0 & 0 & 0 \\
\hline $\begin{array}{l}\text { MUSCULOSKELETAL \& CONNECTIVE } \\
\text { TISSUE }\end{array}$ & 710-739 & 338 & 118 & 352 & 116 \\
\hline CONGENITAL ANOMALIES & \begin{tabular}{|l|}
$740-759$ \\
\end{tabular} & 17 & 0 & 0 & 0 \\
\hline $\begin{array}{l}\text { SYMPTOMS, SIGNS, \& ILL-DEFINED } \\
\text { CONDITIONS }\end{array}$ & 780-799 & 112 & 77 & 137 & 63 \\
\hline INJURY \& POISONING & $800-999$ & 141 & 60 & 289 & 69 \\
\hline
\end{tabular}

\footnotetext{
*Absences with >1 ICD-9-CM code in the same diagnostic category were counted only once. Only those diagnostic categories and gender/job category combinations with at least one occurrence appear in this table.
} 


\section{Kansas City Plant 2007}

Absence Data

Appendix J. Total Number of Calendar Days Absent in Each Diagnostic Category by Gender and Job Category*

\begin{tabular}{|c|c|c|c|c|c|}
\hline & & \multicolumn{4}{|c|}{ Men } \\
\hline & & \multicolumn{3}{|c|}{ Job Category } & \multirow[b]{2}{*}{ TOTAL } \\
\hline & & Security and Fire & Crafts & Line Operators & \\
\hline Diagnostic Category & ICD-9-CM Code & \multirow[b]{2}{*}{29} & \multirow[b]{2}{*}{0} & \multirow[b]{2}{*}{0} & \multirow[b]{2}{*}{42} \\
\hline INFECTIOUS \& PARASITIC DISEASES (DIS) & 001-139 & & & & \\
\hline MALIGNANT NEOPLASMS & $140-208,230-234$ & 0 & 0 & 0 & 89 \\
\hline BENIGN \& UNCERTAIN NEOPLASMS & 210-229, 235-239 & 0 & 0 & 0 & 31 \\
\hline ENDOCRINE/METABOLIC/IMMUNITY & $240-279$ & 0 & 0 & 0 & 241 \\
\hline BLOOD \& BLOOD-FORMING ORGANS & 280-289 & 0 & 148 & 0 & 166 \\
\hline MENTAL DISORDERS & 290-319 & 0 & 0 & 0 & 48 \\
\hline NERVOUS SYSTEM (NS) \& SENSE ORGANS & $320-389$ & 0 & 2 & 2 & 204 \\
\hline CIRCULATORY SYSTEM & $390-459$ & 108 & 3 & 20 & 679 \\
\hline RESPIRATORY SYSTEM & 460-519 & 0 & 30 & 11 & 95 \\
\hline DIGESTIVE SYSTEM & 520-579 & 0 & 34 & 35 & 511 \\
\hline GENITOURINARY SYSTEM & $580-629$ & 29 & 0 & 6 & 75 \\
\hline SKIN \& SUBCUTANEOUS TISSUE & 680-709 & 0 & 0 & 0 & 13 \\
\hline $\begin{array}{l}\text { MUSCULOSKELETAL \& CONNECTIVE } \\
\text { TISSUE }\end{array}$ & 710-739 & 114 & 162 & 43 & 1,243 \\
\hline CONGENITAL ANOMALIES & $740-759$ & 0 & 0 & 0 & 17 \\
\hline $\begin{array}{l}\text { SYMPTOMS, SIGNS, \& ILL-DEFINED } \\
\text { CONDITIONS }\end{array}$ & 780-799 & 4 & 25 & 18 & 436 \\
\hline INJURY \& POISONING & 800-999 & 28 & 157 & 153 & 897 \\
\hline
\end{tabular}

\footnotetext{
*Absences with >1 ICD-9-CM code in the same diagnostic category were counted only once. Only those diagnostic categories and gender/job category combinations with at least one occurrence appear in this table.
} 


\section{Kansas City Plant 2007}

Absence Data

Appendix K. Age-Adjusted IIIness and Injury Rates by Diagnostic Category*

Part 1. Men

\begin{tabular}{|c|c|c|c|c|c|}
\hline & & $\begin{array}{l}\text { Number of } \\
\text { Diagnoses }\end{array}$ & $\begin{array}{c}\text { Age-Adjusted } \\
\text { Rate per } 1,000 * *\end{array}$ & \begin{tabular}{c|} 
Lower $95 \%$ \\
Confidence \\
Limit per 1,000
\end{tabular} & $\begin{array}{c}\text { Upper } 95 \% \\
\text { Confidence } \\
\text { Limit per } 1,000\end{array}$ \\
\hline Diagnostic Category & ICD-9-CM Code & & & & \\
\hline INFECTIOUS \& PARASITIC DISEASES (DIS) & 001-139 & 2 & 0.8 & 0.2 & 3.3 \\
\hline -Other Bacterial Dis & 030-041 & 2 & 0.8 & 0.2 & 3.3 \\
\hline MALIGNANT NEOPLASMS & $140-208,230-234$ & 5 & 1.6 & 0.7 & 4.1 \\
\hline -Bone, Connective Tissue, Skin & $170-173,176$ & 1 & 0.4 & 0.1 & 2.7 \\
\hline -Genitourinary & 179-189 & 1 & 0.5 & 0.1 & 3.2 \\
\hline -Other \& Unspecified Sites & 190, 193-199 & 2 & 0.4 & 0.1 & 1.7 \\
\hline -Nervous System & 191-192 & 1 & 0.4 & 0.1 & 2.7 \\
\hline BENIGN \& UNCERTAIN NEOPLASMS & 210-229, 235-239 & 1 & 0.4 & 0.1 & 2.7 \\
\hline ENDOCRINE/METABOLIC/IMMUNITY & 240-279 & 3 & 0.8 & 0.2 & 2.6 \\
\hline -Thyroid Gland Disorders & $240-246$ & 1 & 0.2 & 0.0 & 1.5 \\
\hline -Other Endocrine Gland Dis & $250-259$ & 1 & 0.2 & 0.0 & 1.5 \\
\hline -Other Metabolic \& Immunity Disorders & $270-279$ & 1 & 0.4 & 0.1 & 2.7 \\
\hline BLOOD \& BLOOD-FORMING ORGANS & $280-289$ & 3 & 1.3 & 0.4 & 4.0 \\
\hline MENTAL DISORDERS & $290-319$ & 3 & 0.8 & 0.2 & 2.6 \\
\hline -Non-Psychotic Disorders & $300-302,306-316$ & 3 & 0.8 & 0.2 & 2.6 \\
\hline NERVOUS SYSTEM (NS) \& SENSE ORGANS & 320-389 & 8 & 3.7 & 1.5 & 9.1 \\
\hline -Other Disorders of Central NS & 340-349 & 1 & 1.5 & 0.2 & 10.3 \\
\hline -Disorders of Peripheral NS & $350-359$ & 5 & 1.8 & 0.7 & 4.5 \\
\hline -Disorders of Eye & $360-379$ & 1 & 0.2 & 0.0 & 1.5 \\
\hline -Dis of Ear \& Mastoid & 380-389 & 1 & 0.2 & 0.0 & 1.5 \\
\hline CIRCULATORY SYSTEM & $390-459$ & 17 & 6.9 & 3.6 & 13.2 \\
\hline -Ischemic Heart Dis & $410-414$ & 9 & 2.5 & 1.2 & 5.0 \\
\hline -Other Heart Dis & $420-429$ & 4 & 3.5 & 1.1 & 11.2 \\
\hline -Cerebrovascular Dis & 430-438 & 1 & 0.2 & 0.0 & 1.5 \\
\hline -Dis of Arteries \& Capillaries & $440-448$ & 1 & 0.2 & 0.0 & 1.5 \\
\hline -Dis of Veins, Lymphatics, Other & 451-459 & 2 & 0.4 & 0.1 & 1.7 \\
\hline RESPIRATORY SYSTEM & $460-519$ & 6 & 4.5 & 1.7 & 11.7 \\
\hline -Acute Respiratory Infections & $460-466$ & 1 & 1.5 & 0.2 & 10.3 \\
\hline -Other Dis Upper Respiratory Tract & $470-478$ & 2 & 0.8 & 0.2 & 3.0 \\
\hline -Pneumonia \& Influenza & 480-487 & 3 & 2.3 & 0.6 & 8.8 \\
\hline DIGESTIVE SYSTEM & $520-579$ & 32 & 13.4 & 8.5 & 21.0 \\
\hline -Esophagus, Stomach, Duodenum & $530-537$ & 5 & 1.2 & 0.5 & 3.0 \\
\hline -Hernia & $550-553$ & 14 & 6.7 & 3.3 & 13.3 \\
\hline -Enteritis, Colitis & \begin{tabular}{|l|}
$555-558$ \\
\end{tabular} & 1 & 0.5 & 0.1 & 3.2 \\
\hline -Other Intestinal Dis & $560-569$ & 5 & 2.6 & 0.8 & 8.5 \\
\hline
\end{tabular}

(Continued)

*Only those diagnostic categories with at least one occurrence appear in this table.

**Standardized to age distribution of 2000 U.S. population. 


\section{Kansas City Plant 2007}

Absence Data

Appendix K. Age-Adjusted IIIness and Injury Rates by Diagnostic Category*

Part 1. Men

\begin{tabular}{|c|c|c|c|c|c|}
\hline & & $\begin{array}{l}\text { Number of } \\
\text { Diagnoses }\end{array}$ & $\begin{array}{c}\text { Age-Adjusted } \\
\text { Rate per } 1,000 * *\end{array}$ & $\begin{array}{c}\text { Lower } 95 \% \\
\text { Confidence } \\
\text { Limit per } 1,000\end{array}$ & $\begin{array}{c}\text { Upper } 95 \% \\
\text { Confidence } \\
\text { Limit per } 1,000\end{array}$ \\
\hline Diagnostic Category & ICD-9-CM Code & \multirow[b]{2}{*}{7} & \multirow[b]{2}{*}{2.4} & \multirow[b]{2}{*}{1.1} & \multirow[b]{2}{*}{5.1} \\
\hline -Other Digestive Dis & $570-579$ & & & & \\
\hline GENITOURINARY SYSTEM & $580-629$ & 7 & 3.7 & 1.5 & 9.3 \\
\hline -Other Urinary Dis & $590-599$ & 2 & 0.9 & 0.2 & 3.6 \\
\hline -Male Genital Organ Dis & $600-608$ & 5 & 2.8 & 0.9 & 8.7 \\
\hline SKIN \& SUBCUTANEOUS TISSUE & $680-709$ & 3 & 1.1 & 0.4 & 3.5 \\
\hline -Infections & $680-686$ & 3 & 1.1 & 0.4 & 3.5 \\
\hline $\begin{array}{l}\text { MUSCULOSKELETAL \& CONNECTIVE } \\
\text { TISSUE }\end{array}$ & $710-739$ & 42 & 16.4 & 10.9 & 24.7 \\
\hline -Arthropathies & $710-719$ & 15 & 6.0 & 3.2 & 11.2 \\
\hline -Dorsopathies & 720-724 & 16 & 6.4 & 3.2 & 12.8 \\
\hline -Rheumatism, Excluding Back & $725-729$ & 10 & 3.9 & 1.6 & 9.0 \\
\hline -Other Dis \& Acquired Deformities & $730-739$ & 1 & 0.2 & 0.0 & 1.5 \\
\hline CONGENITAL ANOMALIES & $740-759$ & 1 & 0.4 & 0.1 & 2.7 \\
\hline $\begin{array}{l}\text { SYMPTOMS, SIGNS, \& ILL-DEFINED } \\
\text { CONDITIONS }\end{array}$ & 780-799 & 22 & 10.7 & 6.3 & 18.3 \\
\hline -Symptoms & $780-789$ & 19 & 9.7 & 5.5 & 17.3 \\
\hline -Non-Specific Abnormal Findings & $790-796$ & 2 & 0.6 & 0.1 & 2.5 \\
\hline -Ill-Defined \& Unknown Causes & 797-799 & 1 & 0.4 & 0.1 & 2.7 \\
\hline INJURY \& POISONING & \begin{tabular}{|l|}
$800-999$ \\
\end{tabular} & 32 & 17.6 & 11.1 & 27.9 \\
\hline -Fracture - Skull & $800-804$ & 2 & 0.4 & 0.1 & 1.7 \\
\hline -Fracture - Upper Limb & 810-819 & 2 & 0.9 & 0.2 & 3.6 \\
\hline -Fracture - Lower Limb & $820-829$ & 1 & 0.4 & 0.1 & 2.7 \\
\hline -Dislocation & 830-839 & 3 & 0.8 & 0.2 & 2.6 \\
\hline -Sprains \& Strains - Other & $840-845,848$ & 10 & 4.8 & 2.0 & 11.5 \\
\hline -Internal Injury - Thorax, Abdomen, Pelvis & $860-869$ & 2 & 0.6 & 0.1 & 2.5 \\
\hline -Open Wound - Head, Neck, Trunk & 870-879 & 1 & 0.4 & 0.1 & 2.7 \\
\hline -Late Effects of Accident & 905-909 & 3 & 3.1 & 0.9 & 11.4 \\
\hline -Superficial Injury & 910-919 & 1 & 0.5 & 0.1 & 3.2 \\
\hline -Contusion & 920-924 & 1 & 0.2 & 0.0 & 1.5 \\
\hline -Burns & $940-949$ & 1 & 1.5 & 0.2 & 10.3 \\
\hline -Complications \& Unspecified Injuries & 958-959 & 3 & 3.3 & 0.9 & 11.4 \\
\hline -Complications of Surgical/Medical Care & 996-999 & 2 & 0.8 & 0.2 & 3.3 \\
\hline Total & & 187 & 84.1 & 69.6 & 101.5 \\
\hline
\end{tabular}

* Only those diagnostic categories with at least one occurrence appear in this table.

**Standardized to age distribution of 2000 U.S. population. 


\section{Kansas City Plant 2007}

\section{Absence Data}

Appendix K. Age-Adjusted Illness and Injury Rates by Diagnostic Category*

Part 2. Women

\begin{tabular}{|c|c|c|c|c|c|}
\hline & & $\begin{array}{l}\text { Number of } \\
\text { Diagnoses }\end{array}$ & $\begin{array}{c}\text { Age-Adjusted } \\
\text { Rate per } 1,000 * *\end{array}$ & $\begin{array}{c}\text { Lower } 95 \% \\
\text { Confidence } \\
\text { Limit per 1,000 }\end{array}$ & $\begin{array}{c}\text { Upper } 95 \% \\
\text { Confidence } \\
\text { Limit per 1,000 }\end{array}$ \\
\hline Diagnostic Category & ICD-9-CM Code & & & & \\
\hline INFECTIOUS \& PARASITIC DISEASES (DIS) & 001-139 & 1 & 0.6 & 0.1 & 4.6 \\
\hline -Viral Dis with Exanthem & 050-057 & 1 & 0.6 & 0.1 & 4.6 \\
\hline BENIGN \& UNCERTAIN NEOPLASMS & 210-229, 235-239 & 1 & 0.6 & 0.1 & 4.6 \\
\hline ENDOCRINE/METABOLIC/IMMUNITY & 240-279 & 2 & 1.8 & 0.4 & 7.7 \\
\hline -Thyroid Gland Disorders & $240-246$ & 1 & 0.6 & 0.1 & 4.6 \\
\hline -Other Metabolic \& Immunity Disorders & $270-279$ & 1 & 1.2 & 0.2 & 8.3 \\
\hline BLOOD \& BLOOD-FORMING ORGANS & $280-289$ & 2 & 1.8 & 0.4 & 7.7 \\
\hline MENTAL DISORDERS & 290-319 & 1 & 1.2 & 0.2 & 8.3 \\
\hline -Non-Psychotic Disorders & $300-302,306-316$ & 1 & 1.2 & 0.2 & 8.3 \\
\hline NERVOUS SYSTEM (NS) \& SENSE ORGANS & 320-389 & 6 & 6.2 & 2.6 & 14.8 \\
\hline -Disorders of Peripheral NS & $350-359$ & 4 & 3.5 & 1.2 & 10.3 \\
\hline -Disorders of Eye & $360-379$ & 1 & 1.6 & 0.2 & 11.2 \\
\hline -Dis of Ear \& Mastoid & 380-389 & 1 & 1.2 & 0.2 & 8.3 \\
\hline CIRCULATORY SYSTEM & $390-459$ & 7 & 6.6 & 3.1 & 14.2 \\
\hline -Ischemic Heart Dis & $410-414$ & 1 & 0.6 & 0.1 & 4.6 \\
\hline -Dis of Pulmonary Circulation & $415-417$ & 1 & 1.2 & 0.2 & 8.3 \\
\hline -Other Heart Dis & $420-429$ & 2 & 1.8 & 0.4 & 7.7 \\
\hline -Dis of Veins, Lymphatics, Other & 451-459 & 3 & 3.0 & 0.9 & 9.6 \\
\hline RESPIRATORY SYSTEM & $460-519$ & 14 & 15.3 & 7.8 & 29.8 \\
\hline -Acute Respiratory Infections & $460-466$ & 2 & 1.3 & 0.3 & 5.2 \\
\hline -Other Dis Upper Respiratory Tract & $470-478$ & 5 & 6.6 & 1.9 & 22.9 \\
\hline -Pneumonia \& Influenza & $480-487$ & 2 & 2.2 & 0.5 & 10.0 \\
\hline -Chronic Obstructive Dis & $490-496$ & 4 & 4.0 & 1.4 & 11.5 \\
\hline -Other Respiratory Dis & $510-519$ & 1 & 1.2 & 0.2 & 8.3 \\
\hline DIGESTIVE SYSTEM & $520-579$ & 1 & 1.2 & 0.2 & 8.3 \\
\hline -Appendicitis & $540-543$ & 1 & 1.2 & 0.2 & 8.3 \\
\hline GENITOURINARY SYSTEM & $580-629$ & 7 & 9.3 & 4.3 & 19.9 \\
\hline -Other Urinary Dis & $590-599$ & 2 & 3.1 & 0.8 & 12.6 \\
\hline -Other Female Disorders & 617-629 & 5 & 6.1 & 2.5 & 15.2 \\
\hline SKIN \& SUBCUTANEOUS TISSUE & 680-709 & 5 & 4.3 & 1.7 & 10.7 \\
\hline -Infections & $680-686$ & 2 & 1.3 & 0.3 & 5.2 \\
\hline -Other Inflammatory Conditions & $690-698$ & 2 & 2.3 & 0.6 & 9.3 \\
\hline -Other & \begin{tabular}{|l|}
$700-709$ \\
\end{tabular} & 1 & 0.6 & 0.1 & 4.6 \\
\hline $\begin{array}{l}\text { MUSCULOSKELETAL \& CONNECTIVE } \\
\text { TISSUE }\end{array}$ & $710-739$ & 27 & 26.2 & 17.5 & 39.2 \\
\hline -Arthropathies & $710-719$ & 9 & 10.2 & 5.1 & 20.2 \\
\hline
\end{tabular}

(Continued)

* Only those diagnostic categories with at least one occurrence appear in this table.

**Standardized to age distribution of 2000 U.S. population. 


\section{Kansas City Plant 2007}

Absence Data

Appendix K. Age-Adjusted Illness and Injury Rates by Diagnostic Category*

Part 2. Women

\begin{tabular}{|c|c|c|c|c|c|}
\hline & & $\begin{array}{l}\text { Number of } \\
\text { Diagnoses }\end{array}$ & $\begin{array}{c}\text { Age-Adjusted } \\
\text { Rate per } 1,000 * *\end{array}$ & \begin{tabular}{|c|} 
Lower $95 \%$ \\
Confidence \\
Limit per 1,000
\end{tabular} & $\begin{array}{c}\text { Upper } 95 \% \\
\text { Confidence } \\
\text { Limit per 1,000 }\end{array}$ \\
\hline Diagnostic Category & ICD-9-CM Code & \multirow[b]{2}{*}{4} & \multirow[b]{2}{*}{3.5} & \multirow[b]{2}{*}{1.2} & \multirow[b]{2}{*}{10.3} \\
\hline -Dorsopathies & $720-724$ & & & & \\
\hline -Rheumatism, Excluding Back & 725-729 & 10 & 9.1 & 4.7 & 17.3 \\
\hline -Other Dis \& Acquired Deformities & $730-739$ & 4 & 3.5 & 1.2 & 10.3 \\
\hline CONGENITAL ANOMALIES & $740-759$ & 1 & 1.6 & 0.2 & 11.2 \\
\hline $\begin{array}{l}\text { SYMPTOMS, SIGNS, \& ILL-DEFINED } \\
\text { CONDITIONS }\end{array}$ & $780-799$ & 12 & 14.8 & 6.7 & 32.3 \\
\hline -Symptoms & $780-789$ & 10 & 8.5 & 4.5 & 16.3 \\
\hline -Non-Specific Abnormal Findings & $790-796$ & 2 & 6.2 & 1.2 & 31.9 \\
\hline INJURY \& POISONING & 800-999 & 12 & 11.6 & 6.3 & 21.3 \\
\hline -Fracture - Skull & $800-804$ & 1 & 0.6 & 0.1 & 4.6 \\
\hline -Fracture - Lower Limb & $820-829$ & 3 & 3.4 & 1.0 & 11.2 \\
\hline -Dislocation & $830-839$ & 1 & 1.6 & 0.2 & 11.2 \\
\hline -Sprains \& Strains - Other & $840-845,848$ & 4 & 4.0 & 1.4 & 11.5 \\
\hline -Late Effects of Accident & $905-909$ & 1 & 0.6 & 0.1 & 4.6 \\
\hline -Burns & 940-949 & 1 & 0.6 & 0.1 & 4.6 \\
\hline -Complications of Surgical/Medical Care & 996-999 & 1 & 0.6 & 0.1 & 4.6 \\
\hline Total & & 99 & 103.0 & 81.9 & 129.6 \\
\hline
\end{tabular}

* Only those diagnostic categories with at least one occurrence appear in this table.

**Standardized to age distribution of 2000 U.S. population. 


\section{Kansas City Plant 2007}

\section{Absence Data}

Appendix K. Age-Adjusted IIIness and Injury Rates by Diagnostic Category*

Part 3. Men and Women

\begin{tabular}{|c|c|c|c|c|c|}
\hline & & $\begin{array}{c}\text { Number of } \\
\text { Diagnoses }\end{array}$ & $\begin{array}{c}\text { Age-Adjusted } \\
\text { Rate per } 1,000 * *\end{array}$ & \begin{tabular}{|c|} 
Lower $95 \%$ \\
Confidence \\
Limit per 1,000
\end{tabular} & $\begin{array}{c}\text { Upper } 95 \% \\
\text { Confidence } \\
\text { Limit per 1,000 }\end{array}$ \\
\hline Diagnostic Category & ICD-9-CM Code & & & & \\
\hline INFECTIOUS \& PARASITIC DISEASES (DIS) & 001-139 & 3 & 0.8 & 0.2 & 2.6 \\
\hline -Other Bacterial Dis & 030-041 & 2 & 0.6 & 0.2 & 2.6 \\
\hline -Viral Dis with Exanthem & 050-057 & 1 & 0.2 & 0.0 & 1.1 \\
\hline MALIGNANT NEOPLASMS & $140-208,230-234$ & 5 & 1.3 & 0.5 & 3.2 \\
\hline -Bone, Connective Tissue, Skin & $170-173,176$ & 1 & 0.3 & 0.0 & 2.0 \\
\hline -Genitourinary & 179-189 & 1 & 0.4 & 0.1 & 2.6 \\
\hline -Other \& Unspecified Sites & 190, 193-199 & 2 & 0.3 & 0.1 & 1.3 \\
\hline -Nervous System & 191-192 & 1 & 0.3 & 0.0 & 2.0 \\
\hline BENIGN \& UNCERTAIN NEOPLASMS & 210-229, 235-239 & 2 & 0.4 & 0.1 & 1.9 \\
\hline ENDOCRINE/METABOLIC/IMMUNITY & 240-279 & 5 & 1.1 & 0.4 & 2.6 \\
\hline -Thyroid Gland Disorders & $240-246$ & 2 & 0.3 & 0.1 & $\overline{1.3}$ \\
\hline -Other Endocrine Gland Dis & 250-259 & 1 & 0.2 & 0.0 & 1.1 \\
\hline -Other Metabolic \& Immunity Disorders & 270-279 & 2 & 0.6 & 0.1 & 2.3 \\
\hline BLOOD \& BLOOD-FORMING ORGANS & $280-289$ & 5 & 1.5 & 0.6 & 3.6 \\
\hline MENTAL DISORDERS & 290-319 & 4 & 0.9 & 0.3 & 2.5 \\
\hline -Non-Psychotic Disorders & $300-302,306-316$ & 4 & 0.9 & 0.3 & 2.5 \\
\hline NERVOUS SYSTEM (NS) \& SENSE ORGANS & 320-389 & 14 & 4.4 & 2.2 & 8.5 \\
\hline -Other Disorders of Central NS & 340-349 & 1 & 1.1 & 0.2 & 8.0 \\
\hline -Disorders of Peripheral NS & 350-359 & 9 & 2.3 & 1.1 & 4.6 \\
\hline -Disorders of Eye & $360-379$ & 2 & 0.5 & 0.1 & 2.3 \\
\hline -Dis of Ear \& Mastoid & 380-389 & 2 & 0.4 & 0.1 & 1.9 \\
\hline CIRCULATORY SYSTEM & $390-459$ & 24 & 6.8 & 4.0 & 11.6 \\
\hline -Ischemic Heart Dis & $410-414$ & 10 & 2.1 & 1.1 & 4.0 \\
\hline -Dis of Pulmonary Circulation & $415-417$ & 1 & 0.3 & 0.0 & 2.0 \\
\hline -Other Heart Dis & 420-429 & 6 & 3.1 & 1.1 & 8.5 \\
\hline -Cerebrovascular Dis & $430-438$ & 1 & 0.2 & 0.0 & 1.1 \\
\hline -Dis of Arteries \& Capillaries & $440-448$ & 1 & 0.2 & 0.0 & 1.1 \\
\hline -Dis of Veins, Lymphatics, Other & 451-459 & 5 & 1.1 & 0.4 & 2.6 \\
\hline RESPIRATORY SYSTEM & $460-519$ & 20 & 7.2 & 4.0 & 12.9 \\
\hline -Acute Respiratory Infections & $460-466$ & 3 & 1.4 & 0.3 & 6.8 \\
\hline -Other Dis Upper Respiratory Tract & $470-478$ & 7 & 2.2 & 0.8 & 6.1 \\
\hline -Pneumonia \& Influenza & $480-487$ & 5 & 2.3 & 0.8 & 6.8 \\
\hline -Chronic Obstructive Dis & $490-496$ & 4 & 1.0 & 0.3 & 2.8 \\
\hline -Other Respiratory Dis & $510-519$ & 1 & 0.3 & 0.0 & 2.0 \\
\hline DIGESTIVE SYSTEM & $520-579$ & 33 & 10.6 & 6.8 & 16.5 \\
\hline
\end{tabular}

(Continued)

* Only those diagnostic categories with at least one occurrence appear in this table.

**Standardized to age distribution of 2000 U.S. population. 


\section{Kansas City Plant 2007}

\section{Absence Data}

Appendix K. Age-Adjusted IIIness and Injury Rates by Diagnostic Category*

Part 3. Men and Women

\begin{tabular}{|c|c|c|c|c|c|}
\hline & & $\begin{array}{l}\text { Number of } \\
\text { Diagnoses }\end{array}$ & $\begin{array}{c}\text { Age-Adjusted } \\
\text { Rate per } 1,000 * *\end{array}$ & $\begin{array}{c}\text { Lower } 95 \% \\
\text { Confidence } \\
\text { Limit per 1,000 }\end{array}$ & $\begin{array}{c}\text { Upper } 95 \% \\
\text { Confidence } \\
\text { Limit per 1,000 }\end{array}$ \\
\hline Diagnostic Category & ICD-9-CM Code & \multirow[b]{2}{*}{5} & \multirow[b]{2}{*}{0.9} & \multirow[b]{2}{*}{0.4} & \multirow[b]{2}{*}{2.3} \\
\hline -Esophagus, Stomach, Duodenum & $530-537$ & & & & \\
\hline -Appendicitis & $540-543$ & 1 & 0.3 & 0.0 & 2.0 \\
\hline -Hernia & $550-553$ & 14 & 5.2 & 2.6 & 10.3 \\
\hline -Enteritis, Colitis & $555-558$ & 1 & 0.4 & 0.1 & 2.6 \\
\hline -Other Intestinal Dis & $560-569$ & 5 & 2.0 & 0.6 & $\overline{6.6}$ \\
\hline -Other Digestive Dis & $570-579$ & 7 & 1.8 & 0.8 & 3.9 \\
\hline GENITOURINARY SYSTEM & $580-629$ & 14 & 5.1 & 2.7 & 9.4 \\
\hline -Other Urinary Dis & $590-599$ & 4 & 1.5 & 0.5 & 3.9 \\
\hline -Male Genital Organ Dis & $600-608$ & 5 & 2.2 & 0.7 & 6.7 \\
\hline -Other Female Disorders & $617-629$ & 5 & 1.5 & 0.6 & 3.6 \\
\hline SKIN \& SUBCUTANEOUS TISSUE & $680-709$ & 8 & 1.9 & 0.9 & 3.9 \\
\hline -Infections & $680-686$ & 5 & 1.2 & 0.5 & 2.9 \\
\hline -Other Inflammatory Conditions & 690-698 & 2 & 0.6 & 0.1 & 2.3 \\
\hline -Other & 700-709 & 1 & 0.2 & 0.0 & 1.1 \\
\hline $\begin{array}{l}\text { MUSCULOSKELETAL \& CONNECTIVE } \\
\text { TISSUE }\end{array}$ & $710-739$ & 69 & 19.0 & 14.0 & 25.7 \\
\hline -Arthropathies & $710-719$ & 24 & 7.0 & 4.4 & 11.3 \\
\hline -Dorsopathies & $720-724$ & 20 & 5.7 & 3.1 & 10.6 \\
\hline -Rheumatism, Excluding Back & 725-729 & 20 & 5.2 & 3.0 & 9.1 \\
\hline -Other Dis \& Acquired Deformities & 730-739 & 5 & 1.0 & 0.4 & 2.6 \\
\hline CONGENITAL ANOMALIES & $740-759$ & 2 & 0.6 & 0.2 & 2.6 \\
\hline $\begin{array}{l}\text { SYMPTOMS, SIGNS, \& ILL-DEFINED } \\
\text { CONDITIONS }\end{array}$ & $780-799$ & 34 & 11.7 & 7.6 & 18.1 \\
\hline -Symptoms & 780-789 & 29 & 9.6 & 6.0 & 15.3 \\
\hline -Non-Specific Abnormal Findings & $790-796$ & 4 & 1.9 & 0.5 & $\overline{6.6}$ \\
\hline -Ill-Defined \& Unknown Causes & 797-799 & 1 & 0.3 & 0.0 & 2.0 \\
\hline INJURY \& POISONING & $800-999$ & 44 & 16.3 & 11.0 & 24.2 \\
\hline -Fracture - Skull & $800-804$ & 3 & 0.5 & 0.2 & 1.5 \\
\hline -Fracture - Upper Limb & 810-819 & 2 & 0.7 & 0.2 & 2.9 \\
\hline -Fracture - Lower Limb & $820-829$ & 4 & 1.1 & 0.4 & 3.0 \\
\hline -Dislocation & $830-839$ & 4 & 1.0 & 0.3 & 2.8 \\
\hline -Sprains \& Strains - Other & $840-845,848$ & 14 & 4.6 & 2.2 & 9.5 \\
\hline -Internal Injury - Thorax, Abdomen, Pelvis & $860-869$ & 2 & 0.4 & 0.1 & 1.9 \\
\hline -Open Wound - Head, Neck, Trunk & $870-879$ & 1 & 0.3 & 0.0 & 2.0 \\
\hline -Late Effects of Accident & 905-909 & 4 & 2.6 & 0.8 & 8.7 \\
\hline -Superficial Injury & $910-919$ & 1 & 0.4 & 0.1 & 2.6 \\
\hline
\end{tabular}

(Continued)

*Only those diagnostic categories with at least one occurrence appear in this table.

**Standardized to age distribution of 2000 U.S. population. 
Kansas City Plant 2007

Absence Data

Appendix K. Age-Adjusted Illness and Injury Rates by Diagnostic Category*

Part 3. Men and Women

\begin{tabular}{|c|c|c|c|c|c|}
\hline & & $\begin{array}{l}\text { Number of } \\
\text { Diagnoses }\end{array}$ & $\begin{array}{c}\text { Age-Adjusted } \\
\text { Rate per } 1,000 * *\end{array}$ & $\begin{array}{c}\text { Lower } 95 \% \\
\text { Confidence } \\
\text { Limit per 1,000 }\end{array}$ & $\begin{array}{c}\text { Upper } 95 \% \\
\text { Confidence } \\
\text { Limit per 1,000 }\end{array}$ \\
\hline Diagnostic Category & ICD-9-CM Code & \multirow[b]{2}{*}{1} & \multirow[b]{2}{*}{0.2} & \multirow[b]{2}{*}{0.0} & \multirow[b]{2}{*}{1.1} \\
\hline -Contusion & $920-924$ & & & & \\
\hline -Burns & 940-949 & 2 & 1.3 & 0.2 & 7.2 \\
\hline -Complications \& Unspecified Injuries & 958-959 & 3 & 2.5 & 0.7 & 8.8 \\
\hline -Complications of Surgical/Medical Care & 996-999 & 3 & 0.8 & 0.2 & 2.6 \\
\hline Total & & 286 & 89.6 & 77.1 & 104.1 \\
\hline
\end{tabular}

*Only those diagnostic categories with at least one occurrence appear in this table.

**Standardized to age distribution of 2000 U.S. population. 
Kansas City Plant 2007

OSHA Data

Appendix L. Number of Workers with at Least One OSHA Event by Gender, Age, and Job Category*

\begin{tabular}{|c|c|c|c|c|c|c|c|c|c|}
\hline \multirow{3}{*}{ Job Category } & \multicolumn{4}{|c|}{ Women } & \multicolumn{4}{|c|}{ Men } & \multirow[b]{3}{*}{ TOTAL } \\
\hline & \multicolumn{3}{|c|}{ Age Group } & \multirow[b]{2}{*}{ TOTAL } & \multicolumn{3}{|c|}{ Age Group } & \multirow[b]{2}{*}{ TOTAL } & \\
\hline & $16-29$ & $40-49$ & $\mathbf{5 0 +}$ & & $16-29$ & $40-49$ & $50+$ & & \\
\hline Professional & 1 & 0 & 1 & 2 & 0 & 0 & 0 & 0 & 2 \\
\hline Administrative Support & 0 & 1 & 1 & 2 & 0 & 0 & 1 & 1 & 3 \\
\hline Technical Support & 0 & 0 & 1 & 1 & 0 & 1 & 2 & 3 & 4 \\
\hline Service & 0 & 0 & 2 & 2 & 1 & 1 & 0 & 2 & 4 \\
\hline Crafts & 0 & 0 & 0 & 0 & 0 & 2 & 5 & 7 & 7 \\
\hline Line Operators & 0 & 0 & 2 & 2 & 0 & 0 & 0 & 0 & 2 \\
\hline TOTAL & 1 & 1 & 7 & 9 & 1 & 4 & 8 & 13 & 22 \\
\hline
\end{tabular}

*Only those job categories and gender/age combinations with at least one OSHA event appear in this table.

Appendix M. Total Number of Workdays Lost or with Restricted Activity from OSHA Events by Gender and Age

\begin{tabular}{|c|c|c|c|c|c|c|c|c|c|}
\hline \multirow[b]{2}{*}{ Age Group } & \multicolumn{3}{|c|}{ Women } & \multicolumn{3}{|c|}{ Men } & \multicolumn{3}{|c|}{ TOTAL } \\
\hline & $\begin{array}{c}\text { Number of } \\
\text { Events }\end{array}$ & \begin{tabular}{|c} 
Days \\
Restricted
\end{tabular} & Days Lost & $\begin{array}{c}\text { Number of } \\
\text { Events }\end{array}$ & $\begin{array}{c}\text { Days } \\
\text { Restricted }\end{array}$ & Days Lost & \begin{tabular}{|c|} 
Number of \\
Events
\end{tabular} & $\begin{array}{c}\text { Days } \\
\text { Restricted }\end{array}$ & Days Lost \\
\hline $16-29$ & 1 & 0 & 0 & 1 & 0 & 0 & 2 & 0 & 0 \\
\hline $40-49$ & 1 & 0 & 0 & 4 & 56 & 0 & 5 & 56 & 0 \\
\hline $50+$ & 7 & 18 & 4 & 8 & 94 & 23 & 15 & 112 & 27 \\
\hline TOTAL & 9 & 18 & 4 & 13 & 150 & 23 & 22 & 168 & 27 \\
\hline
\end{tabular}

Appendix N. Total Number of Workdays Lost or with Restricted Activity from OSHA Events by Gender and Job Category*

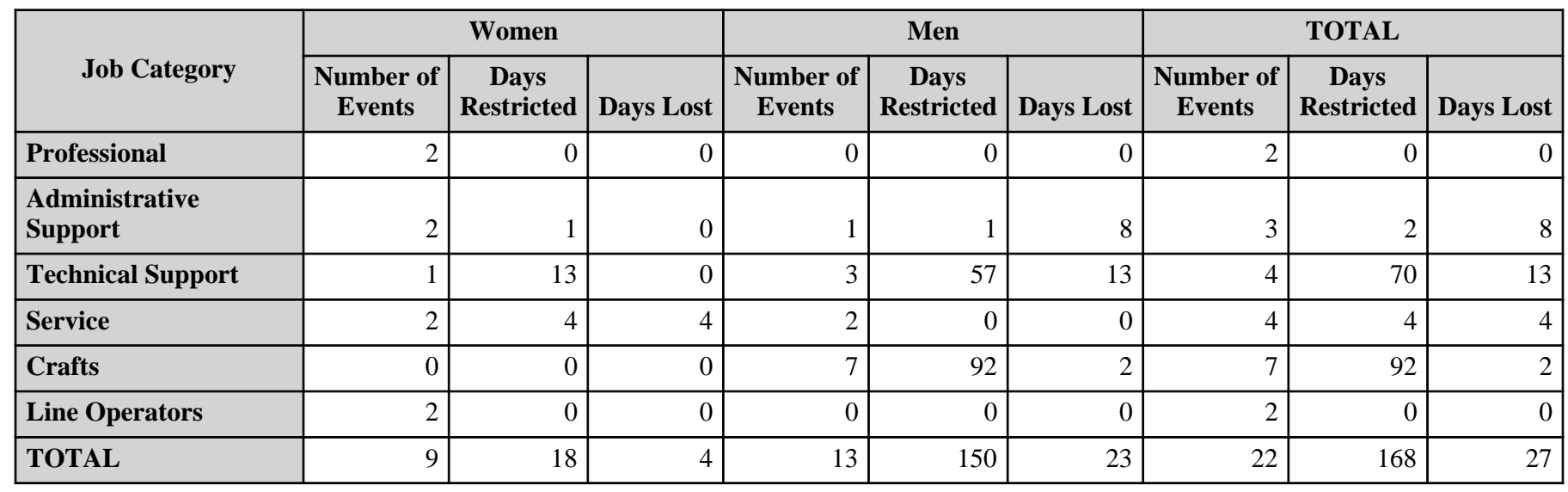

*Only those job categories with at least one OSHA event appear in this table. 


\section{Kansas City Plant 2007}

OSHA Data

Appendix O. Number of Diagnoses in Each Diagnostic Category by Gender and Age*

\begin{tabular}{|c|c|c|c|c|c|}
\hline & & \multicolumn{4}{|c|}{ Women } \\
\hline & & \multicolumn{3}{|c|}{ Age Group } & \multirow[b]{2}{*}{ TOTAL } \\
\hline & & $16-29$ & $40-49$ & $50+$ & \\
\hline Diagnostic Category & ICD-9-CM code & \multirow[b]{2}{*}{0} & \multirow[b]{2}{*}{0} & \multirow[b]{2}{*}{1} & \multirow[b]{2}{*}{1} \\
\hline NERVOUS SYSTEM (NS) \& SENSE ORGANS & $320-389$ & & & & \\
\hline -Disorders of Peripheral NS & $350-359$ & 0 & 0 & 1 & 1 \\
\hline RESPIRATORY SYSTEM & $460-519$ & 0 & 0 & 1 & 1 \\
\hline -Lung Dis from External Agents & $500-508$ & 0 & 0 & 1 & 1 \\
\hline $\begin{array}{l}\text { MUSCULOSKELETAL \& CONNECTIVE } \\
\text { TISSUE }\end{array}$ & $710-739$ & 0 & 0 & 4 & 4 \\
\hline -Arthropathies & 710-719 & 0 & 0 & 2 & 2 \\
\hline -Dorsopathies & $720-724$ & 0 & 0 & 1 & 1 \\
\hline -Rheumatism, Excluding Back & $725-729$ & 0 & 0 & 1 & 1 \\
\hline $\begin{array}{l}\text { SYMPTOMS, SIGNS, \& ILL-DEFINED } \\
\text { CONDITIONS }\end{array}$ & 780-799 & 0 & 0 & 0 & 0 \\
\hline -Symptoms & $780-789$ & 0 & 0 & 0 & 0 \\
\hline INJURY \& POISONING & $800-999$ & 1 & 2 & 9 & 12 \\
\hline -Fracture - Upper Limb & 810-819 & 0 & 1 & 0 & 1 \\
\hline -Fracture - Lower Limb & $820-829$ & 0 & 0 & 2 & 2 \\
\hline -Dislocation & 830-839 & 0 & 0 & 1 & 1 \\
\hline -Sprains \& Strains - Back & 846-847 & 0 & 0 & 1 & 1 \\
\hline -Sprains \& Strains - Other & $840-845,848$ & 1 & 0 & 4 & 5 \\
\hline -Open Wound - Upper Limb & $880-887$ & 0 & 0 & 0 & 0 \\
\hline -Superficial Injury & 910-919 & 0 & 0 & 1 & 1 \\
\hline -Foreign Body Entering Orifice & $930-939$ & 0 & 0 & 0 & 0 \\
\hline -Complications \& Unspecified Injuries & 958-959 & 0 & 1 & 0 & 1 \\
\hline
\end{tabular}

*Only those diagnostic categories and gender/age combinations with at least one OSHA event appear in this table. 


\section{Kansas City Plant 2007}

OSHA Data

Appendix O. Number of Diagnoses in Each Diagnostic Category by Gender and Age*

\begin{tabular}{|c|c|c|c|c|c|c|}
\hline & & \multicolumn{4}{|c|}{ Men } & \multirow[b]{3}{*}{ TOTAL } \\
\hline & & \multicolumn{3}{|c|}{ Age Group } & \multirow[b]{2}{*}{ TOTAL } & \\
\hline & & $16-29$ & $40-49$ & $50+$ & & \\
\hline Diagnostic Category & ICD-9-CM code & & & & & \\
\hline NERVOUS SYSTEM (NS) \& SENSE ORGANS & $320-389$ & 0 & 0 & 0 & 0 & 1 \\
\hline -Disorders of Peripheral NS & $350-359$ & 0 & 0 & 0 & 0 & 1 \\
\hline RESPIRATORY SYSTEM & $460-519$ & 0 & 0 & 0 & 0 & 1 \\
\hline -Lung Dis from External Agents & $500-508$ & 0 & 0 & 0 & 0 & 1 \\
\hline $\begin{array}{l}\text { MUSCULOSKELETAL \& CONNECTIVE } \\
\text { TISSUE }\end{array}$ & 710-739 & 0 & 7 & 7 & 14 & 18 \\
\hline -Arthropathies & $710-719$ & 0 & 1 & 4 & 5 & 7 \\
\hline -Dorsopathies & $720-724$ & 0 & 4 & 3 & 7 & 8 \\
\hline -Rheumatism, Excluding Back & 725-729 & 0 & 2 & 0 & 2 & 3 \\
\hline $\begin{array}{l}\text { SYMPTOMS, SIGNS, \& ILL-DEFINED } \\
\text { CONDITIONS }\end{array}$ & 780-799 & 0 & 1 & 1 & 2 & 2 \\
\hline -Symptoms & 780-789 & 0 & 1 & 1 & 2 & 2 \\
\hline INJURY \& POISONING & $800-999$ & 1 & 2 & 6 & 9 & 21 \\
\hline -Fracture - Upper Limb & 810-819 & 0 & 0 & 1 & 1 & 2 \\
\hline -Fracture - Lower Limb & $820-829$ & 0 & 0 & 0 & 0 & 2 \\
\hline -Dislocation & $830-839$ & 0 & 0 & 0 & 0 & 1 \\
\hline -Sprains \& Strains - Back & 846-847 & 0 & 0 & 0 & 0 & 1 \\
\hline -Sprains \& Strains - Other & $840-845,848$ & 0 & 1 & 2 & 3 & 8 \\
\hline -Open Wound - Upper Limb & $880-887$ & 1 & 1 & 2 & 4 & 4 \\
\hline -Superficial Injury & $910-919$ & 0 & 0 & 0 & 0 & 1 \\
\hline -Foreign Body Entering Orifice & $930-939$ & 0 & 0 & 1 & 1 & 1 \\
\hline -Complications \& Unspecified Injuries & 958-959 & 0 & 0 & 0 & 0 & 1 \\
\hline
\end{tabular}

\begin{tabular}{|c|c|c|c|c|c|c|c|c|c|}
\hline & \multicolumn{4}{|c|}{ Women } & \multicolumn{4}{|c|}{ Men } & \multirow[b]{3}{*}{ TOTAL } \\
\hline & \multicolumn{3}{|c|}{ Age Group } & \multirow[b]{2}{*}{ TOTAL } & \multicolumn{3}{|c|}{ Age Group } & \multirow[b]{2}{*}{ TOTAL } & \\
\hline & $16-29$ & $40-49$ & $50+$ & & $16-29$ & $40-49$ & $50+$ & & \\
\hline Diagnostic Category & \multirow[b]{2}{*}{1} & \multirow[b]{2}{*}{2} & \multirow[b]{2}{*}{15} & \multirow[b]{2}{*}{18} & \multirow[b]{2}{*}{1} & \multirow[b]{2}{*}{10} & \multirow[b]{2}{*}{14} & \multirow[b]{2}{*}{25} & \multirow[b]{2}{*}{43} \\
\hline TOTAL & & & & & & & & & \\
\hline
\end{tabular}

*Only those diagnostic categories and gender/age combinations with at least one OSHA event appear in this table. 


\section{Kansas City Plant 2007}

OSHA Data

Appendix P. Number of Workdays Lost or with Restricted Activity in Each Diagnostic Category by Gender and Age*

\begin{tabular}{|c|c|c|c|c|c|c|c|}
\hline & & \multicolumn{6}{|c|}{ Women } \\
\hline & & \multicolumn{6}{|c|}{ Age Group } \\
\hline & & \multicolumn{2}{|c|}{$16-29$} & \multicolumn{2}{|c|}{$40-49$} & \multicolumn{2}{|c|}{$50+$} \\
\hline & & \begin{tabular}{c|} 
Days \\
Restricted
\end{tabular} & Days Lost & \begin{tabular}{c|} 
Days \\
Restricted
\end{tabular} & Days Lost & \begin{tabular}{|c|} 
Days \\
Restricted
\end{tabular} & Days Lost \\
\hline Diagnostic Category & ICD-9-CM Codes & & & & & & \\
\hline -Disorders of Peripheral NS & $350-359$ & 0 & 0 & 0 & 0 & 1 & 0 \\
\hline -Lung Dis from External Agents & $500-508$ & 0 & 0 & 0 & 0 & 0 & 0 \\
\hline -Arthropathies & $710-719$ & 0 & 0 & 0 & 0 & 4 & 4 \\
\hline -Dorsopathies & $720-724$ & 0 & 0 & 0 & 0 & 0 & 0 \\
\hline -Rheumatism, Excluding Back & $725-729$ & 0 & 0 & 0 & 0 & 0 & 0 \\
\hline -Fracture - Upper Limb & $810-819$ & 0 & 0 & 0 & 0 & 0 & 0 \\
\hline -Fracture - Lower Limb & $820-829$ & 0 & 0 & 0 & 0 & 13 & 0 \\
\hline -Dislocation & $830-839$ & 0 & 0 & 0 & 0 & 4 & 4 \\
\hline -Sprains \& Strains - Back & 846-847 & 0 & 0 & 0 & 0 & 0 & 0 \\
\hline -Sprains \& Strains - Other & $840-845,848$ & 0 & 0 & 0 & 0 & 13 & 0 \\
\hline -Superficial Injury & $910-919$ & 0 & 0 & 0 & 0 & 0 & 0 \\
\hline $\begin{array}{l}\text {-Complications \& Unspecified } \\
\text { Injuries }\end{array}$ & 958-959 & 0 & 0 & 0 & 0 & 0 & 0 \\
\hline
\end{tabular}

\begin{tabular}{|c|c|c|c|c|c|c|c|}
\hline & & \multicolumn{6}{|c|}{ Men } \\
\hline & & \multicolumn{6}{|c|}{ Age Group } \\
\hline & & \multicolumn{2}{|c|}{$16-29$} & \multicolumn{2}{|c|}{$40-49$} & \multicolumn{2}{|c|}{$50+$} \\
\hline & & \begin{tabular}{|c|} 
Days \\
Restricted
\end{tabular} & Days Lost & $\begin{array}{c}\text { Days } \\
\text { Restricted }\end{array}$ & Days Lost & $\begin{array}{c}\text { Days } \\
\text { Restricted }\end{array}$ & Days Lost \\
\hline Diagnostic Category & ICD-9-CM Codes & \multirow[b]{2}{*}{0} & \multirow[b]{2}{*}{0} & \multirow[b]{2}{*}{0} & \multirow[b]{2}{*}{0} & \multirow[b]{2}{*}{22} & \multirow[b]{2}{*}{8} \\
\hline -Arthropathies & $710-719$ & & & & & & \\
\hline -Dorsopathies & $720-724$ & 0 & 0 & 56 & 0 & 0 & 0 \\
\hline -Rheumatism, Excluding Back & 725-729 & 0 & 0 & 56 & 0 & 0 & 0 \\
\hline -Symptoms & 780-789 & 0 & 0 & 56 & 0 & 0 & 0 \\
\hline -Fracture - Upper Limb & $810-819$ & 0 & 0 & 0 & 0 & 71 & 2 \\
\hline -Sprains \& Strains - Other & $840-845,848$ & 0 & 0 & 0 & 0 & 2 & 21 \\
\hline -Open Wound - Upper Limb & $880-887$ & 0 & 0 & 0 & 0 & 72 & 10 \\
\hline -Foreign Body Entering Orifice & 930-939 & 0 & 0 & 0 & 0 & 0 & 0 \\
\hline
\end{tabular}

*OSHA events with >1 ICD-9-CM code in the same diagnostic category were counted only once. Only those diagnostic categories and gender/age combinations with at least one occurrence appear in this table. 
Kansas City Plant 2007

OSHA Data

Appendix Q. Number of Occurrences in Each Accident Category by Gender and Age*

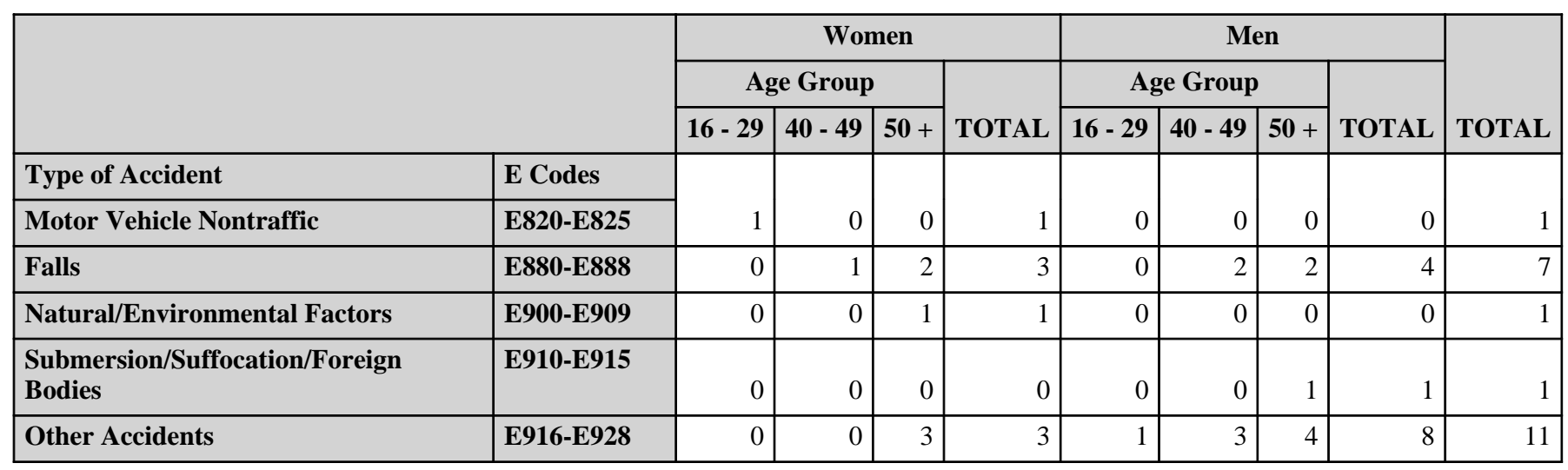

*Only those accident types and gender/age combinations with at least one occurrence appear in this table. 
Kansas City Plant 2007

OSHA Data

Appendix R. Number of Workdays Lost or with Restricted Activity in Each Accident Category by Gender and Age*

\begin{tabular}{|c|c|c|c|c|c|c|c|}
\hline & & \multicolumn{6}{|c|}{ Women } \\
\hline & & \multicolumn{6}{|c|}{ Age Group } \\
\hline & & \multicolumn{2}{|c|}{$16-29$} & \multicolumn{2}{|c|}{$40-49$} & \multicolumn{2}{|c|}{$50+$} \\
\hline & & $\begin{array}{c}\text { Days } \\
\text { Restricted }\end{array}$ & Days Lost & $\begin{array}{c}\text { Days } \\
\text { Restricted }\end{array}$ & Days Lost & $\begin{array}{c}\text { Days } \\
\text { Restricted }\end{array}$ & Days Lost \\
\hline Type of Accident & E Codes & \multirow[b]{2}{*}{0} & \multirow[b]{2}{*}{0} & \multirow[b]{2}{*}{0} & \multirow[b]{2}{*}{0} & \multirow[b]{2}{*}{0} & \multirow[b]{2}{*}{0} \\
\hline Motor Vehicle Nontraffic & E820-E825 & & & & & & \\
\hline Falls & E880-E888 & 0 & 0 & 0 & 0 & 13 & 0 \\
\hline Natural/Environmental Factors & E900-E909 & 0 & 0 & 0 & 0 & 0 & 0 \\
\hline Other Accidents & E916-E928 & 0 & 0 & 0 & 0 & 5 & 4 \\
\hline
\end{tabular}

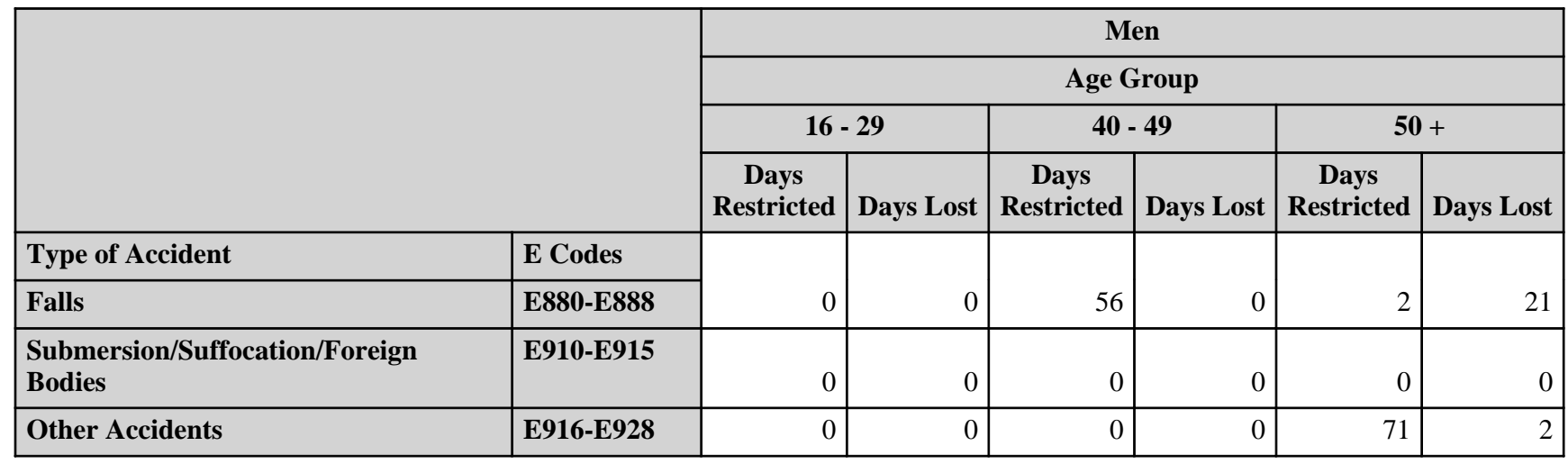

*OSHA events with $>1 \mathrm{E}$ code in the same accident type were counted only once. Only those accident types and gender/age combinations with at least one occurrence appear in this table. 


\section{Kansas City Plant 2007}

OSHA Data

Appendix S. Number of Diagnoses in Each Diagnostic Category by Gender and Job Category*

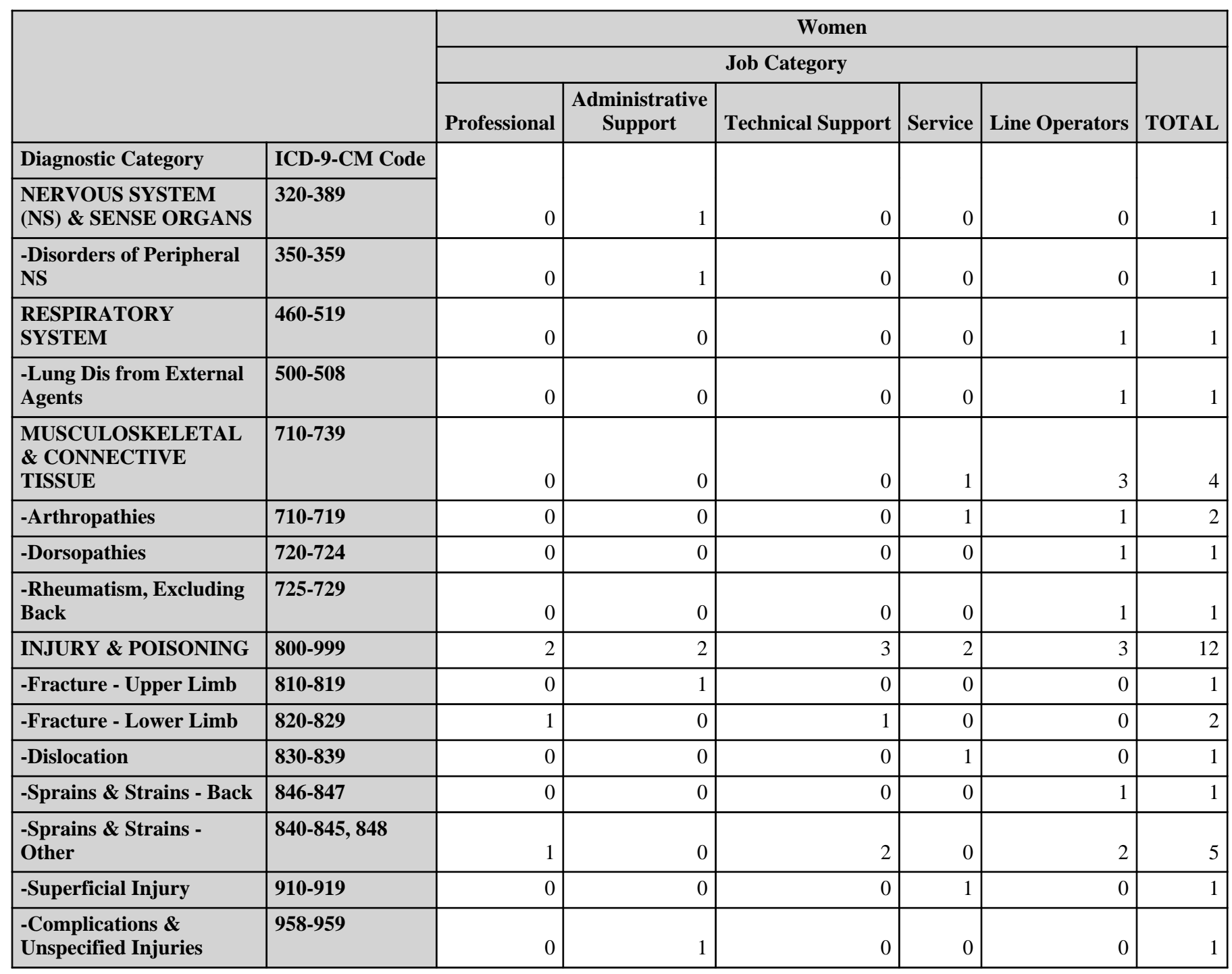

\begin{tabular}{|c|c|c|c|c|c|c|}
\hline & \multicolumn{6}{|c|}{ Women } \\
\hline & \multicolumn{5}{|c|}{ Job Category } & \multirow[b]{2}{*}{ TOTAL } \\
\hline & Professional & $\begin{array}{c}\text { Administrative } \\
\text { Support }\end{array}$ & Technical Support & Service & Line Operators & \\
\hline Diagnostic Category & \multirow[b]{2}{*}{2} & \multirow[b]{2}{*}{3} & \multirow[b]{2}{*}{3} & \multirow[b]{2}{*}{3} & \multirow[b]{2}{*}{7} & \multirow[b]{2}{*}{18} \\
\hline \begin{tabular}{|l} 
TOTAL \\
\end{tabular} & & & & & & \\
\hline
\end{tabular}

*Only those diagnostic categories and gender/job category combinations with at least one occurrence appear in this table. 


\section{Kansas City Plant 2007}

OSHA Data

Appendix S. Number of Diagnoses in Each Diagnostic Category by Gender and Job Category*

\begin{tabular}{|c|c|c|c|c|c|c|}
\hline & & \multicolumn{5}{|c|}{ Men } \\
\hline & & \multicolumn{4}{|c|}{ Job Category } & \multirow[b]{2}{*}{ TOTAL } \\
\hline & & $\begin{array}{l}\text { Administrative } \\
\text { Support }\end{array}$ & Technical Support & Service & Crafts & \\
\hline Diagnostic Category & ICD-9-CM Code & & & & & \\
\hline $\begin{array}{l}\text { MUSCULOSKELETAL } \\
\text { \& CONNECTIVE } \\
\text { TISSUE }\end{array}$ & $710-739$ & 1 & 4 & 2 & 7 & 14 \\
\hline -Arthropathies & $710-719$ & 1 & 0 & 0 & 4 & 5 \\
\hline -Dorsopathies & $720-724$ & 0 & 3 & 2 & 2 & 7 \\
\hline $\begin{array}{l}\text {-Rheumatism, Excluding } \\
\text { Back }\end{array}$ & $725-729$ & 0 & 1 & 0 & 1 & 2 \\
\hline $\begin{array}{l}\text { SYMPTOMS, } \\
\text { SIGNS, \& ILL-DEFINED } \\
\text { CONDITIONS }\end{array}$ & $780-799$ & 0 & 2 & 0 & 0 & 2 \\
\hline -Symptoms & 780-789 & 0 & 2 & 0 & 0 & 2 \\
\hline INJURY \& POISONING & $800-999$ & 2 & 1 & 1 & 5 & 9 \\
\hline -Fracture - Upper Limb & $810-819$ & 0 & 0 & 0 & 1 & 1 \\
\hline $\begin{array}{l}\text {-Sprains \& Strains - } \\
\text { Other }\end{array}$ & $840-845,848$ & 1 & 1 & 0 & 1 & 3 \\
\hline $\begin{array}{l}\text {-Open Wound - Upper } \\
\text { Limb }\end{array}$ & $880-887$ & 1 & 0 & 1 & 2 & 4 \\
\hline $\begin{array}{l}\text {-Foreign Body Entering } \\
\text { Orifice }\end{array}$ & 930-939 & 0 & 0 & 0 & 1 & 1 \\
\hline
\end{tabular}

\begin{tabular}{|c|c|c|c|c|c|}
\hline & \multicolumn{5}{|c|}{ Men } \\
\hline & \multicolumn{4}{|c|}{ Job Category } & \multirow[b]{2}{*}{ TOTAL } \\
\hline & $\begin{array}{l}\text { Administrative } \\
\text { Support }\end{array}$ & Technical Support & Service & Crafts & \\
\hline Diagnostic Category & \multirow[b]{2}{*}{3} & \multirow[b]{2}{*}{7} & \multirow[b]{2}{*}{3} & \multirow[b]{2}{*}{12} & \multirow[b]{2}{*}{25} \\
\hline TOTAL & & & & & \\
\hline
\end{tabular}

*Only those diagnostic categories and gender/job category combinations with at least one occurrence appear in this table. 


\section{Kansas City Plant 2007}

OSHA Data

Appendix T. Number of Workdays Lost or with Restricted Activity in Each Diagnostic Category by Gender and Job Category*

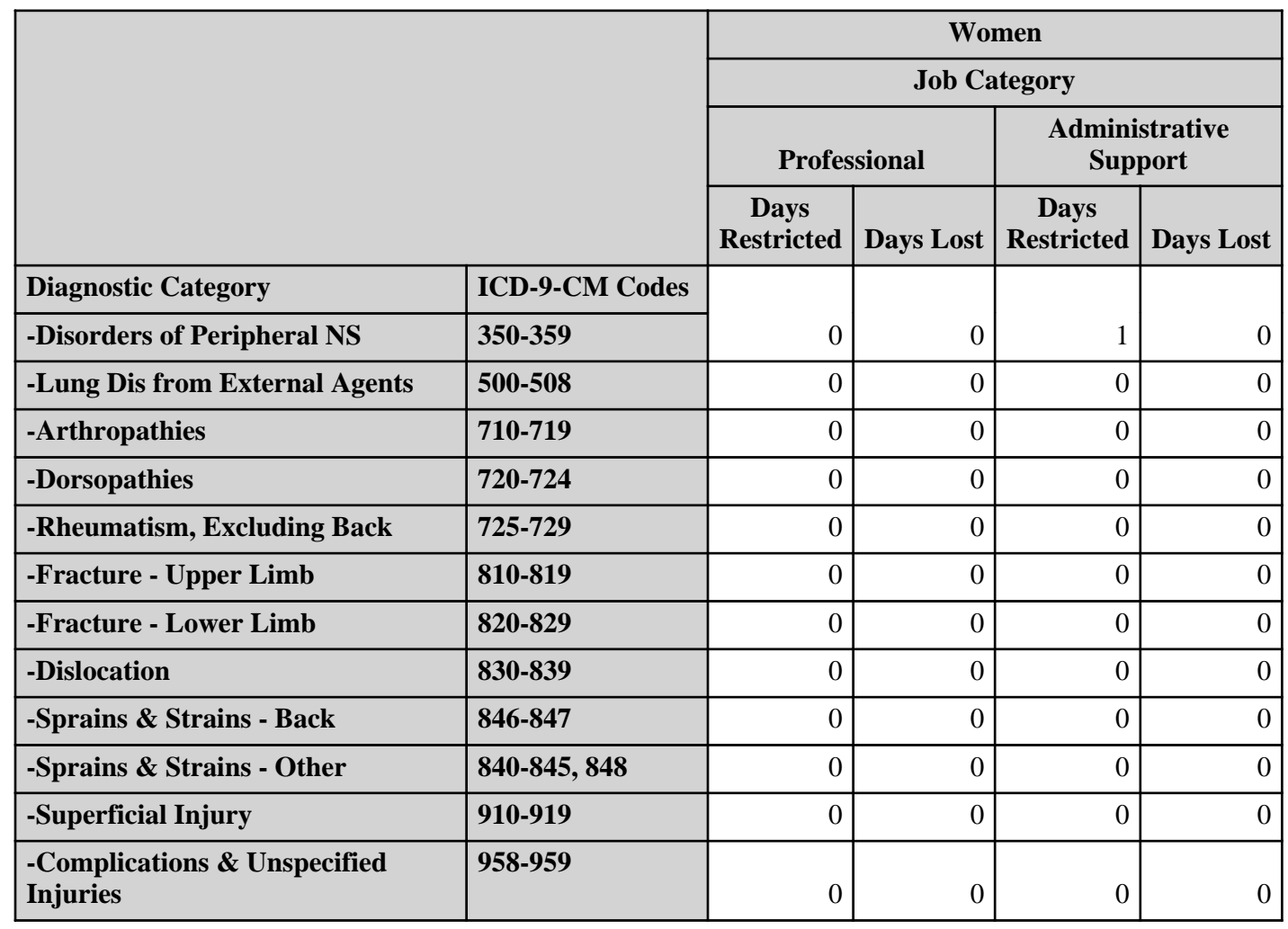

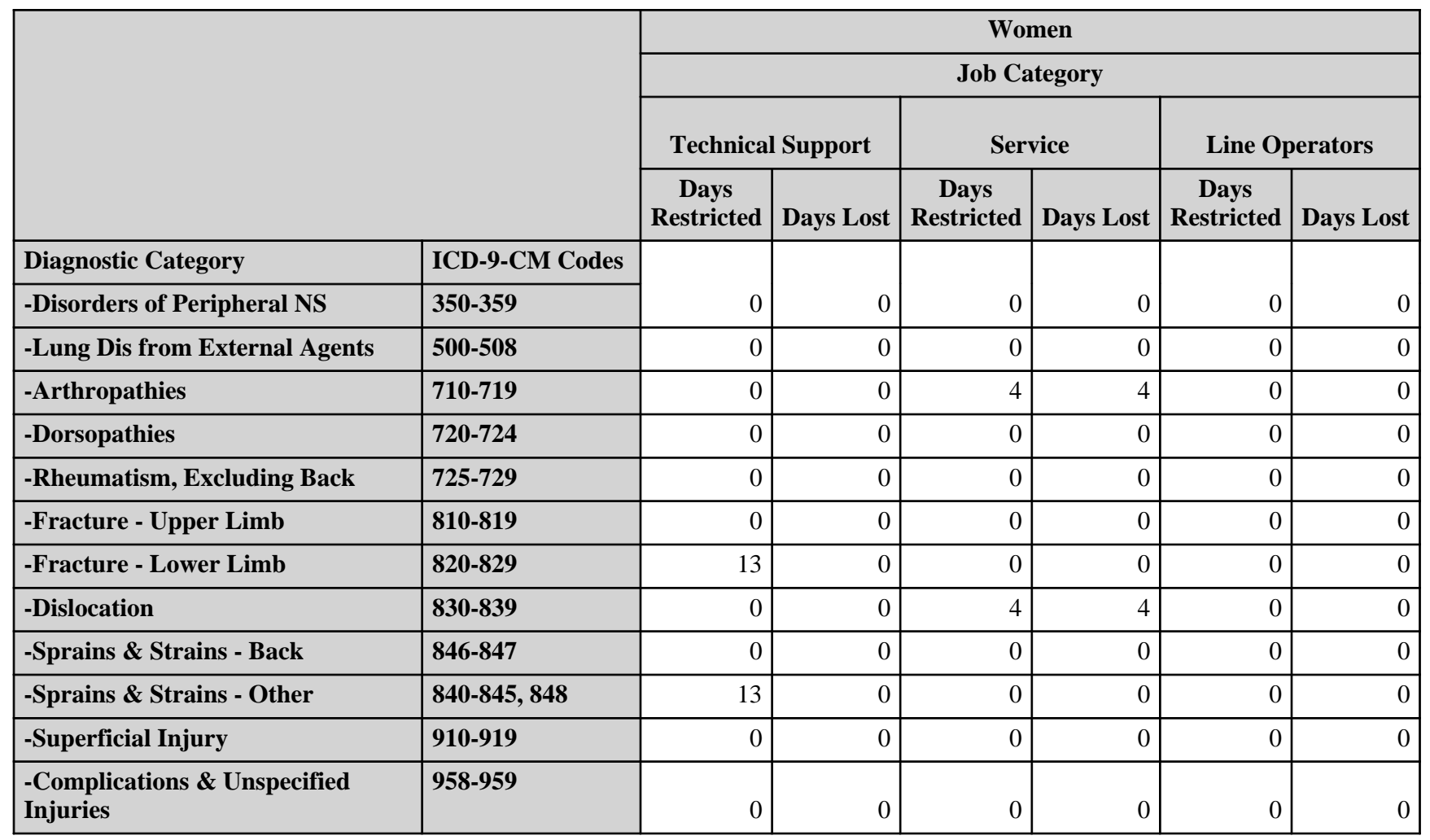

*OSHA events with >1 ICD-9-CM code in the same diagnostic category were counted only once. Only those diagnostic categories and gender/job category combinations with at least one occurrence appear in this table. 
Kansas City Plant 2007

OSHA Data

Appendix T. Number of Workdays Lost or with Restricted Activity in Each Diagnostic Category by Gender and Job Category*

\begin{tabular}{|c|c|c|c|c|c|c|c|c|c|}
\hline & & \multicolumn{8}{|c|}{ Men } \\
\hline & & \multicolumn{8}{|c|}{ Job Category } \\
\hline & & \multicolumn{2}{|c|}{$\begin{array}{c}\text { Administrative } \\
\text { Support }\end{array}$} & \multicolumn{2}{|c|}{$\begin{array}{l}\text { Technical } \\
\text { Support }\end{array}$} & \multicolumn{2}{|c|}{ Service } & \multicolumn{2}{|c|}{ Crafts } \\
\hline & & $\begin{array}{c}\text { Days } \\
\text { Restricted }\end{array}$ & $\begin{array}{l}\text { Days } \\
\text { Lost }\end{array}$ & $\begin{array}{c}\text { Days } \\
\text { Restricted }\end{array}$ & $\begin{array}{l}\text { Days } \\
\text { Lost }\end{array}$ & $\begin{array}{c}\text { Days } \\
\text { Restricted }\end{array}$ & $\begin{array}{l}\text { Days } \\
\text { Lost }\end{array}$ & $\begin{array}{c}\text { Days } \\
\text { Restricted }\end{array}$ & $\begin{array}{l}\text { Days } \\
\text { Lost }\end{array}$ \\
\hline Diagnostic Category & ICD-9-CM Codes & \multirow[b]{2}{*}{1} & \multirow[b]{2}{*}{8} & \multirow[b]{2}{*}{0} & \multirow[b]{2}{*}{0} & \multirow[b]{2}{*}{0} & \multirow[b]{2}{*}{0} & \multirow[b]{2}{*}{21} & \multirow[b]{2}{*}{0} \\
\hline -Arthropathies & $710-719$ & & & & & & & & \\
\hline -Dorsopathies & \begin{tabular}{|l|}
$720-724$ \\
\end{tabular} & 0 & 0 & 56 & 0 & 0 & 0 & 0 & 0 \\
\hline -Rheumatism, Excluding Back & $725-729$ & 0 & 0 & 56 & 0 & 0 & 0 & 0 & 0 \\
\hline -Symptoms & \begin{tabular}{|l|}
$780-789$ \\
\end{tabular} & 0 & 0 & 56 & 0 & 0 & 0 & 0 & 0 \\
\hline -Fracture - Upper Limb & 810-819 & 0 & 0 & 0 & 0 & 0 & 0 & 71 & 2 \\
\hline -Sprains \& Strains - Other & $840-845,848$ & 1 & 8 & 1 & 13 & 0 & 0 & 0 & 0 \\
\hline -Open Wound - Upper Limb & $880-887$ & 1 & 8 & 0 & 0 & 0 & 0 & 71 & 2 \\
\hline -Foreign Body Entering Orifice & 930-939 & 0 & 0 & 0 & 0 & 0 & \begin{tabular}{l|l}
0 \\
\end{tabular} & 0 & 0 \\
\hline
\end{tabular}

*OSHA events with >1 ICD-9-CM code in the same diagnostic category were counted only once. Only those diagnostic categories and gender/job category combinations with at least one occurrence appear in this table. 
Kansas City Plant 2007

OSHA Data

Appendix U. Number of Occurrences in Each Accident Category by Gender and Job Category*

\begin{tabular}{|c|c|c|c|c|c|c|c|}
\hline & & \multicolumn{6}{|c|}{ Women } \\
\hline & & \multicolumn{5}{|c|}{ Job Category } & \multirow[b]{2}{*}{ TOTAL } \\
\hline & & Professional & \begin{tabular}{|c|} 
Administrative \\
Support
\end{tabular} & $\begin{array}{l}\text { Technical } \\
\text { Support }\end{array}$ & Service & $\begin{array}{c}\text { Line } \\
\text { Operators }\end{array}$ & \\
\hline Type of Accident & E CODES & \multirow[b]{2}{*}{1} & \multirow[b]{2}{*}{0} & \multirow[b]{2}{*}{0} & \multirow[b]{2}{*}{0} & \multirow[b]{2}{*}{0} & \multirow[b]{2}{*}{1} \\
\hline Motor Vehicle Nontraffic & E820-E825 & & & & & & \\
\hline Falls & E880-E888 & 1 & 1 & 1 & 0 & 0 & 3 \\
\hline Natural/Environmental Factors & E900-E909 & 0 & 0 & 0 & 1 & 0 & 1 \\
\hline Other Accidents & E916-E928 & 0 & 1 & 0 & 1 & 1 & 3 \\
\hline
\end{tabular}

\begin{tabular}{|c|c|c|c|c|c|c|}
\hline & & \multicolumn{5}{|c|}{ Men } \\
\hline & & \multicolumn{4}{|c|}{ Job Category } & \multirow[b]{2}{*}{ TOTAL } \\
\hline & & $\begin{array}{l}\text { Administrative } \\
\text { Support }\end{array}$ & \begin{tabular}{l|} 
Technical \\
Support
\end{tabular} & Service & Crafts & \\
\hline Type of Accident & E CODES & \multirow[b]{2}{*}{1} & \multirow[b]{2}{*}{2} & \multirow[b]{2}{*}{0} & \multirow[b]{2}{*}{1} & \multirow[b]{2}{*}{4} \\
\hline Falls & E880-E888 & & & & & \\
\hline $\begin{array}{l}\text { Submersion/Suffocation/Foreign } \\
\text { Bodies }\end{array}$ & E910-E915 & 0 & 0 & 0 & 1 & 1 \\
\hline Other Accidents & E916-E928 & 0 & 1 & 2 & 5 & 8 \\
\hline
\end{tabular}

*Only those accident types and gender/job category combinations with at least one occurrence appear in this table. 
Kansas City Plant 2007

OSHA Data

Appendix V. Number of Workdays Lost or with Restricted Activity in Each Accident Category by Gender and Job Category*

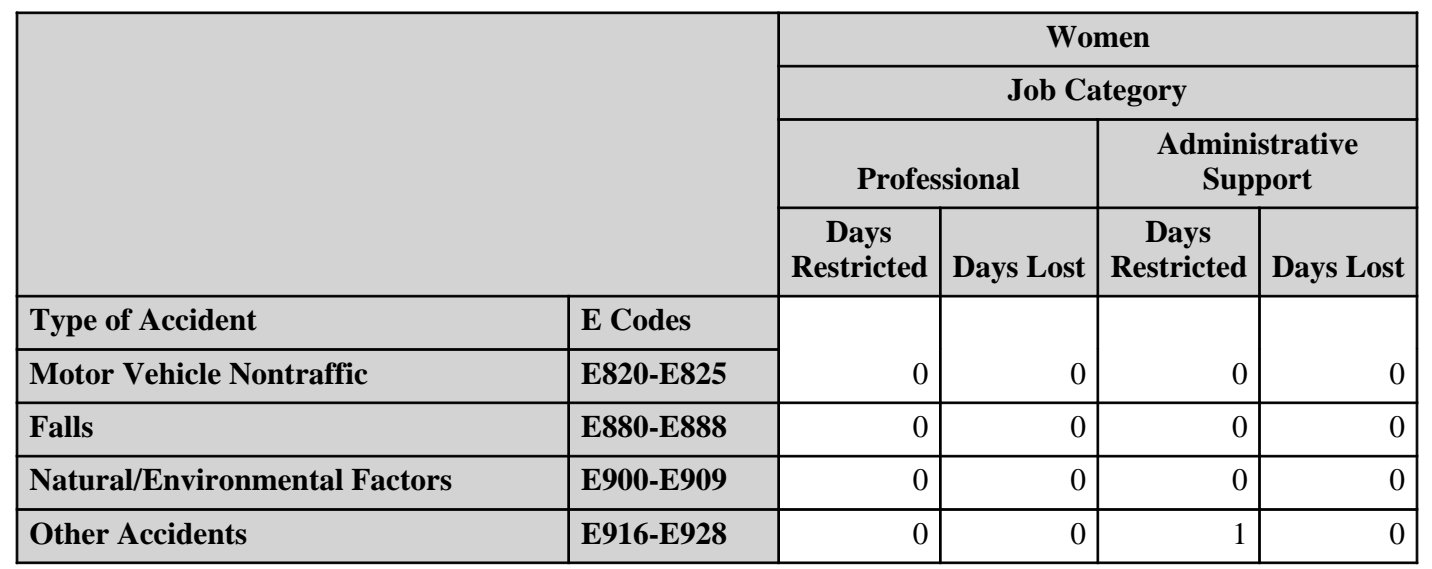

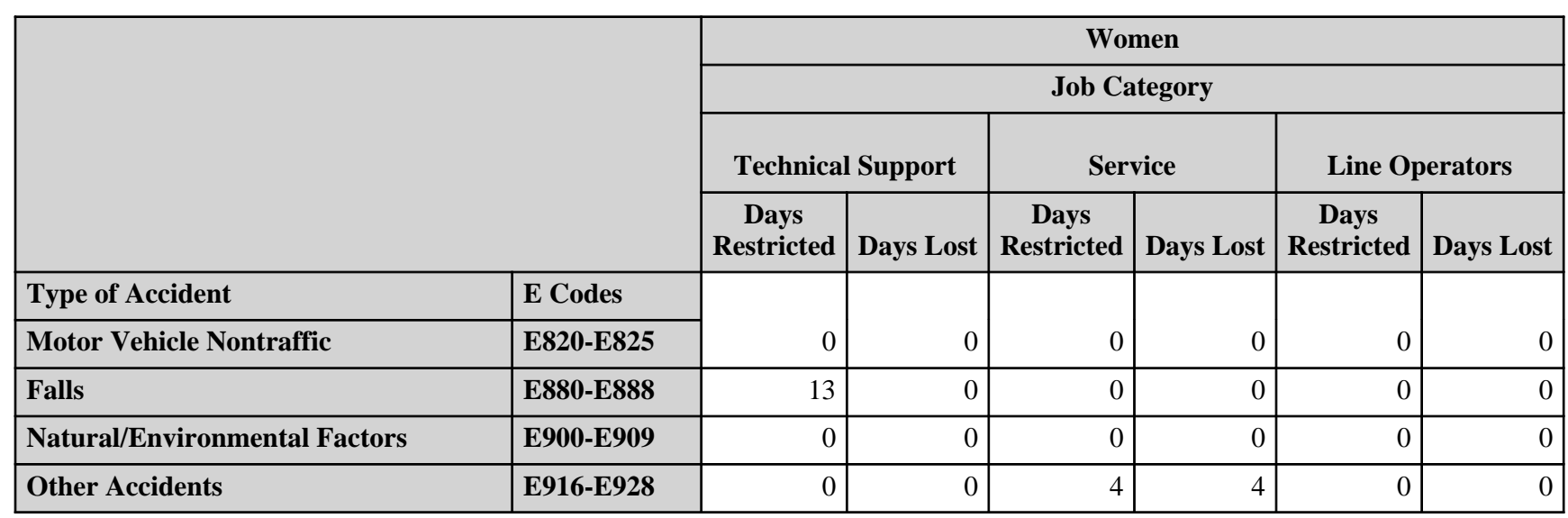

\begin{tabular}{|c|c|c|c|c|c|c|c|c|c|}
\hline & \multicolumn{8}{|c|}{ Men } \\
\hline & & \multicolumn{8}{|c|}{ Job Category } \\
\hline & & \multicolumn{2}{|c|}{$\begin{array}{l}\text { Administrative } \\
\text { Support }\end{array}$} & \multicolumn{2}{|c|}{$\begin{array}{l}\text { Technical } \\
\text { Support }\end{array}$} & \multicolumn{2}{|c|}{ Service } & \multicolumn{2}{|c|}{ Crafts } \\
\hline & & \begin{tabular}{|c|} 
Days \\
Restricted
\end{tabular} & $\begin{array}{l}\text { Days } \\
\text { Lost }\end{array}$ & \begin{tabular}{|c|} 
Days \\
Restricted
\end{tabular} & $\begin{array}{l}\text { Days } \\
\text { Lost }\end{array}$ & \begin{tabular}{|c|} 
Days \\
Restricted
\end{tabular} & $\begin{array}{l}\text { Days } \\
\text { Lost }\end{array}$ & \begin{tabular}{|c|} 
Days \\
Restricted
\end{tabular} & $\begin{array}{l}\text { Days } \\
\text { Lost }\end{array}$ \\
\hline Type of Accident & E Codes & & & & & & & & \\
\hline Falls & E880-E888 & 1 & 8 & 57 & 13 & 0 & 0 & 0 & 0 \\
\hline $\begin{array}{l}\text { Submersion/Suffocation/Foreign } \\
\text { Bodies }\end{array}$ & E910-E915 & 0 & 0 & 0 & 0 & 0 & 0 & 0 & 0 \\
\hline Other Accidents & E916-E928 & 0 & 0 & 0 & 0 & 0 & 0 & 71 & 2 \\
\hline
\end{tabular}

*OSHA events with $>1 \mathrm{E}$ code in the same accident type were counted only once. Only those accident types and gender/job category combinations with at least one occurrence appear in this table. 
Kansas City Plant 2007

OSHA Data

Appendix W. Age-Adjusted OSHA Illness and Injury Rates by Diagnostic Category*

Part 1. Men

\begin{tabular}{|c|c|c|c|c|c|}
\hline & & $\begin{array}{l}\text { Number of } \\
\text { Diagnoses }\end{array}$ & $\begin{array}{l}\text { Age-Adjusted } \\
\text { Rate per } 1,000 * *\end{array}$ & $\begin{array}{c}\text { Lower } 95 \% \\
\text { Confidence } \\
\text { Limit per } 1,000\end{array}$ & $\begin{array}{c}\text { Upper } 95 \% \\
\text { Confidence } \\
\text { Limit per } 1,000\end{array}$ \\
\hline Diagnostic Category & ICD-9-CM Code & & & & \\
\hline $\begin{array}{l}\text { MUSCULOSKELETAL \& CONNECTIVE } \\
\text { TISSUE }\end{array}$ & $710-739$ & 14 & 4.6 & 2.7 & 7.9 \\
\hline -Arthropathies & $710-719$ & 5 & 1.2 & 0.5 & 3.0 \\
\hline -Dorsopathies & $720-724$ & 7 & 2.6 & 1.2 & 5.6 \\
\hline -Rheumatism, Excluding Back & 725-729 & 2 & 0.8 & 0.2 & 3.0 \\
\hline $\begin{array}{l}\text { SYMPTOMS, SIGNS, \& ILL-DEFINED } \\
\text { CONDITIONS }\end{array}$ & 780-799 & 2 & 0.8 & 0.2 & 3.3 \\
\hline -Symptoms & $780-789$ & 2 & 0.8 & 0.2 & 3.3 \\
\hline INJURY \& POISONING & $800-999$ & 9 & 3.4 & 1.4 & 8.5 \\
\hline -Fracture - Upper Limb & 810-819 & 1 & 0.2 & 0.0 & 1.5 \\
\hline -Sprains \& Strains - Other & $840-845,848$ & 3 & 0.8 & 0.2 & 2.6 \\
\hline -Open Wound - Upper Limb & 880-887 & 4 & 2.2 & 0.6 & 8.2 \\
\hline -Foreign Body Entering Orifice & 930-939 & 1 & 0.2 & 0.0 & 1.5 \\
\hline Total & & 25 & 8.9 & 5.6 & 14.2 \\
\hline
\end{tabular}

* Only those diagnostic categories with at least one occurrence appear in this table.

**Standardized to age distribution of 2000 U.S. population. 
Kansas City Plant 2007

OSHA Data

Appendix W. Age-Adjusted OSHA Illness and Injury Rates by Diagnostic Category*

Part 2. Women

\begin{tabular}{|c|c|c|c|c|c|}
\hline & & $\begin{array}{l}\text { Number of } \\
\text { Diagnoses }\end{array}$ & $\begin{array}{c}\text { Age-Adjusted } \\
\text { Rate per } 1,000 * *\end{array}$ & $\begin{array}{c}\text { Lower } 95 \% \\
\text { Confidence } \\
\text { Limit per } 1,000\end{array}$ & $\begin{array}{c}\text { Upper 95\% } \\
\text { Confidence } \\
\text { Limit per 1,000 }\end{array}$ \\
\hline Diagnostic Category & ICD-9-CM Code & \multirow[b]{2}{*}{1} & \multirow[b]{2}{*}{0.6} & \multirow[b]{2}{*}{0.1} & \multirow[b]{2}{*}{4.6} \\
\hline NERVOUS SYSTEM (NS) \& SENSE ORGANS & 320-389 & & & & \\
\hline -Disorders of Peripheral NS & $350-359$ & 1 & 0.6 & 0.1 & $\overline{4.6}$ \\
\hline RESPIRATORY SYSTEM & $460-519$ & 1 & 0.6 & 0.1 & 4.6 \\
\hline -Lung Dis from External Agents & \begin{tabular}{|l|}
$500-508$ \\
\end{tabular} & 1 & 0.6 & 0.1 & $\overline{4.6}$ \\
\hline $\begin{array}{l}\text { MUSCULOSKELETAL \& CONNECTIVE } \\
\text { TISSUE }\end{array}$ & $710-739$ & 4 & 3.5 & 1.2 & 10.3 \\
\hline -Arthropathies & $710-719$ & 2 & 2.2 & 0.5 & 10.0 \\
\hline -Dorsopathies & $720-724$ & 1 & 0.6 & 0.1 & 4.6 \\
\hline -Rheumatism, Excluding Back & $725-729$ & 1 & 0.6 & 0.1 & $\overline{4.6}$ \\
\hline INJURY \& POISONING & $800-999$ & 12 & 13.0 & 6.2 & 27.4 \\
\hline -Fracture - Upper Limb & 810-819 & 1 & 1.2 & 0.2 & 8.3 \\
\hline -Fracture - Lower Limb & $820-829$ & 2 & 1.3 & 0.3 & 5.2 \\
\hline -Dislocation & $830-839$ & 1 & 1.6 & 0.2 & 11.2 \\
\hline -Sprains \& Strains - Back & 846-847 & 1 & 0.6 & 0.1 & 4.6 \\
\hline -Sprains \& Strains - Other & $840-845,848$ & 5 & 6.6 & 1.9 & 22.9 \\
\hline -Superficial Injury & $910-919$ & 1 & 0.6 & 0.1 & $\overline{4.6}$ \\
\hline -Complications of Surgical/Medical Care & 996-999 & 1 & 1.2 & 0.2 & 8.3 \\
\hline Total & & 18 & 17.8 & 9.9 & 32.2 \\
\hline
\end{tabular}

* Only those diagnostic categories with at least one occurrence appear in this table.

**Standardized to age distribution of 2000 U.S. population. 
Kansas City Plant 2007

OSHA Data

Appendix W. Age-Adjusted OSHA Illness and Injury Rates by Diagnostic Category*

Part 3. Men and Women

\begin{tabular}{|c|c|c|c|c|c|}
\hline & & $\begin{array}{l}\text { Number of } \\
\text { Diagnoses }\end{array}$ & $\begin{array}{c}\text { Age-Adjusted } \\
\text { Rate per 1,000** }\end{array}$ & \begin{tabular}{|c|} 
Lower $95 \%$ \\
Confidence \\
Limit per 1,000
\end{tabular} & $\begin{array}{c}\text { Upper 95\% } \\
\text { Confidence } \\
\text { Limit per 1,000 }\end{array}$ \\
\hline Diagnostic Category & ICD-9-CM Code & \multirow[b]{2}{*}{1} & \multirow[b]{2}{*}{0.2} & \multirow[b]{2}{*}{0.0} & \multirow[b]{2}{*}{1.1} \\
\hline NERVOUS SYSTEM (NS) \& SENSE ORGANS & 320-389 & & & & \\
\hline -Disorders of Peripheral NS & $350-359$ & 1 & 0.2 & 0.0 & 1.1 \\
\hline RESPIRATORY SYSTEM & $460-519$ & 1 & 0.2 & 0.0 & 1.1 \\
\hline -Lung Dis from External Agents & $500-508$ & 1 & 0.2 & 0.0 & 1.1 \\
\hline $\begin{array}{l}\text { MUSCULOSKELETAL \& CONNECTIVE } \\
\text { TISSUE }\end{array}$ & $710-739$ & 18 & 4.4 & 2.7 & 7.1 \\
\hline -Arthropathies & $710-719$ & 7 & 1.5 & 0.7 & 3.2 \\
\hline -Dorsopathies & $720-724$ & 8 & 2.2 & 1.1 & 4.5 \\
\hline -Rheumatism, Excluding Back & 725-729 & 3 & 0.7 & 0.2 & 2.3 \\
\hline $\begin{array}{l}\text { SYMPTOMS, SIGNS, \& ILL-DEFINED } \\
\text { CONDITIONS }\end{array}$ & $780-799$ & 2 & 0.6 & 0.2 & 2.6 \\
\hline -Symptoms & 780-789 & 2 & 0.6 & 0.2 & 2.6 \\
\hline INJURY \& POISONING & $800-999$ & 21 & 5.8 & 3.3 & 10.3 \\
\hline -Fracture - Upper Limb & 810-819 & 2 & 0.4 & 0.1 & 1.9 \\
\hline -Fracture - Lower Limb & 820-829 & 2 & 0.3 & 0.1 & 1.3 \\
\hline -Dislocation & 830-839 & 1 & 0.4 & 0.1 & 2.6 \\
\hline -Sprains \& Strains - Back & 846-847 & 1 & 0.2 & 0.0 & 1.1 \\
\hline -Sprains \& Strains - Other & $840-845,848$ & 8 & 2.3 & 0.9 & 6.0 \\
\hline -Open Wound - Upper Limb & $880-887$ & 4 & 1.6 & 0.4 & 6.0 \\
\hline -Superficial Injury & $910-919$ & 1 & 0.2 & 0.0 & 1.1 \\
\hline -Foreign Body Entering Orifice & $930-939$ & 1 & 0.2 & 0.0 & 1.1 \\
\hline -Complications of Surgical/Medical Care & 996-999 & 1 & 0.3 & 0.0 & 2.0 \\
\hline Total & & 43 & 11.2 & 7.7 & 16.1 \\
\hline
\end{tabular}

* Only those diagnostic categories with at least one occurrence appear in this table.

**Standardized to age distribution of 2000 U.S. population. 